\title{
THE FUNCTIONAL DETERMINANT OF A FOUR-DIMENSIONAL BOUNDARY VALUE PROBLEM
}

\author{
THOMAS P. BRANSON AND PETER B. GILKEY
}

\begin{abstract}
Working on four-dimensional manifolds with boundary, we consider, elliptic boundary value problems $(A, B), A$ being the interior and $B$ the boundary operator. These problems $(A, B)$ should be valued in a tensorspinor bundle; should depend in a universal way on a Riemannian metric $g$ and be formally selfadjoint; should behave in an appropriate way under conformal change $g \rightarrow \Omega^{2} g, \Omega$ a smooth positive function; and the leading symbol of $A$ should be positive definite. We view the functional determinant $\operatorname{det} A_{B}$ of such a problem as a functional on a conformal class $\left\{\Omega^{2} g\right\}$, and develop a formula for the quotient of the determinant at $\Omega^{2} g$ by that at $g$. (Analogous formulas are known to be intimately related to physical string theories in dimension two, and to sharp inequalities of borderline Sobolev embedding and Moser-Trudinger types for the boundariless case in even dimensions.) When the determinant in a background metric $g_{0}$ is explicitly computable, the result is a formula for the determinant at each metric $\Omega^{2} g_{0}$ (not just a quotient of determinants). For example, we compute the functional determinants of the Dirichlet and Robin (conformally covariant Neumann) problems for the Laplacian in the ball $B^{4}$, using our general quotient formulas in the case of the conformal Laplacian, together with an explicit computation on the hemisphere $H^{4}$.
\end{abstract}

\section{INTRODUCTION}

The functional determinant $\operatorname{det} A$ of an elliptic differential operator $A$ is important in quantum field theory because it provides a regularization of the functional integral. Originally of interest on four-dimensional manifolds, these objects have recently been intensively studied by physicists and mathematicians in two dimensions, in connection with string theory, the isospectral problem, and uniformization problems. In each of these applications, the operator $A=A_{g}$ should be built naturally from a Riemannian metric $g$ on a compact manifold $M$ (and possibly some related extra information, like spin structure), and one is concerned with det $A_{g}$ as a functional on the cone $\{g\}$ of Riemannian metrics on $M$, or more precisely, the quotient of $\{g\}$ by the action of the diffeomorphism group $\operatorname{Diffeo}(M)$. A key point has been the behavior of the determinant under conformal change of $g$; that is, replacement of $g$ by $\Omega^{2} g$, where $\Omega$ is

Received by the editors October 28, 1992 and, in revised form, September 21, 1993.

1991 Mathematics Subject Classification. Primary 58G25, 58G20.

Research of T. Branson partially supported by the N.S.F. and by the Danish Research Council. Research of P. Gilkey partially supported by the N.S.F. and by the Max Planck Institute for Mathematics (Bonn). 
a smooth positive function. The idea is that if $A$ has reasonable conformal behavior, then the behavior of $\operatorname{det} A$ should be predictable, much as the behavior of the fundamental solution of $A$ is. In the two-dimensional case, this thinking gets one quite far, as the quotient of $\{g\}$ by the groups $\operatorname{Diffeo}(M)$ and $C_{+}^{\infty}(M)$ (the positive functions $\Omega$ acting by $g \mapsto \Omega^{2} g$ ) is a finite-parameter object; see, e.g., [O, OPS1-2]. Since diffeomorphisms act on conformal factors $\Omega$, this quotient has the form $\mathscr{G}=\{g\} /\left(\operatorname{Diffeo}(M) \ltimes C_{+}^{\infty}(M)\right)$; i.e., the total group is a semidirect product. In dimensions three and higher, $\mathscr{G}$ is much larger, and in particular is in no sense a finite-parameter object. Even though it is not yet clear how one would go about tracking the behavior of the functional determinant as the metric $g$ cuts across conformal classes, it seems timely to return to four dimensions, and, inspired by two-dimensional successes, at least handle the behavior of $\operatorname{det} A$ as a functional on a conformal class $C_{+}^{\infty}(M) \cdot g$.

For compact manifolds without boundary, some results are already in place. For a computation in connection with Yang-Mills theory on four-manifolds, see [CT]. In [BØ3], Branson and Ørsted derived a formula for the functional determinant of a strongly elliptic differential operator, with reasonable conformal properties, over a Riemannian four-manifold without boundary; this is analogous to the much-studied Polyakov formulas on two-manifolds. Branson, Chang, and Yang [BCY] used these formulas to study the isospectral and extremal (uniformization) problems in four dimensions, trying to get analogues of the two-dimensional results of Onofri [O] and of Osgood, Phillips, and Sarnak [OPS1-2]. The conformal behavior of the functional determinant in dimension two is intimately related to the Moser-Trudinger inequality, which expresses the continuity of the embedding $L_{1}^{2} \hookrightarrow e^{L}$ of the Sobolev class $L_{1}^{2}$ in the Orlicz class $e^{L}$. Specifically, let $d \xi$ and $\Delta$ be the normalized measure and Laplacian of the round metric $g_{0}$ on $S^{2}$. Let $\omega \in C^{\infty}\left(S^{2}\right)$, and let $\bar{\omega}=\int \omega d \xi$. The Moser-Trudinger inequality states that if $\omega \in C^{\infty}\left(S^{2}\right)$,

$$
(\Delta \omega, w)_{L^{2}\left(S^{2}, d \xi\right)}-\log \int_{S^{2}} e^{2(\omega-\bar{\omega})} d \xi \geq 0
$$

with equality if and only if the metric $e^{2 \omega} g_{0}$ is the pullback of $g_{0}$ by a Möbius transformation. (0.1) may be regarded as a limit of borderline Sobolev inequalities $L_{\nu}^{2} \hookrightarrow L^{2 /(1-\nu)}$ (where $L^{p}$ is the usual Lebesgue class) as $\nu \uparrow 1$, or as $m \downarrow 2$. A scale-invariant version of the logarithm of the functional determinant of the Laplacian is, up to a constant factor, the quantity on the left in (0.1). In general dimension $m$, the borderline Sobolev inequalities correspond to the embeddings $L_{\nu}^{2} \hookrightarrow L^{2 m /(m-2 \nu)}$. On $S^{m}$, the limiting case is an inequality of Moser-Trudinger type recently introduced by Beckner [Be], and describes an embedding $L_{m / 2}^{2} \hookrightarrow e^{L}$. Specifically, for a certain $m$ th-order pseudodifferential operator $P_{m}$ with the same leading symbol as $\Delta^{m / 2}$,

$$
\left(P_{m} \omega, w\right)_{L^{2}\left(S^{m}, d \xi\right)}-\log \int_{S^{m}} e^{m(\omega-\bar{\omega})} d \xi \geq 0,
$$

with equality if and only if the metric $e^{2 \omega} g_{0}$ is the pullback of $g_{0}$ by a conformal transformation. $P_{m}$ is a differential operator if $m$ is even. (See [Br3, Remark 2.23] for a description of $P_{m}$.) In dimension four, [B $\varnothing 3$ and BCY] show that the logarithm of the functional determinant is a linear combination of two 
terms, say $a_{1} \mathscr{A}_{1}+a_{2} \mathscr{A}_{2} . \mathscr{A}_{2}$ is the left side of (0.2), which describes the embedding $L_{2}^{2} \hookrightarrow e^{L}$, and $\mathscr{A}_{1}$ is a quantity which similarly describes the "ordinary" borderline Sobolev embedding $L_{1}^{2} \hookrightarrow L^{4}$. Up to normalization, the $L_{2}^{2} \hookrightarrow e^{L}$ and $L_{1}^{2} \hookrightarrow L^{4}$ terms are connected by one coupling constant $a=a_{1} / a_{2}$. The two inequalities "work together" (the quantities asserted to be nonnegative do not appear with opposite signs) if and only if $a \geq 0 . a=a[A]$ depends on the elliptic operator $A$ whose functional determinant we are studying; for example, $A$ could be the conformal Laplacian $Y$ or the square $\nabla^{2}$ of the Dirac operator $\not$. But $a[A]$ is universal in the sense of being independent of the particular manifold and Riemannian metric; indeed, the number $a[A]$ can be computed from a knowledge of the heat invariants, which are similarly universal. Fortunately, $a[Y]$ and $a\left[\not{ }^{2}\right]$ are positive; this make possible, among other things, the extremal results of [BCY, $\S 5]$ for the log-determinant on $S^{4}$. On more general manifolds, the study of the functional determinant is based on an inequality of Adams [A], which describes the embeddings $L_{m / 2}^{2} \hookrightarrow e^{L}$ for domains in $\mathbb{R}^{m}$; see [BCY] for details

In this paper, we begin the extension of this program to four-dimensional manifolds with boundary, inspired by the complete two-dimensional treatment of [OPS1-2]. As will become clear, this is qualitatively harder than the boundariless case, but still tractable on a conceptual as well as computational level. (The three-dimensional case is not particularly interesting for manifolds without boundary, as the functional determinant is quite rigid conformally in odd dimensions; see $\S 2$. The boundary value version of this three-dimensional problem is more interesting, though not as rich as the four-dimensional theory.) It would seem that an effective treatment of isospectral and extremal problems in the boundary-value case would have to await a theory of boundary-value inequalities of Moser-Trudinger type; we note that an excellent theory of sharp borderline Sobolev inequalities is already in place [E1-2].

We shall need to be precise about three types of assumptions on the elliptic operator $A$ and the boundary operator $B$ which define our problem: (1) analytic assumptions, i.e., the strength of the ellipticity needed; (2) naturality assumptions; and (3) conformal assumptions. Since we wish to invoke invarianttheoretic properties of local spectral invariants associated to $(A, B)$, specifically the heat invariants, we need to know that $(A, B)$ enjoys suitable invariance properties; this is the rationale behind (2). (3) makes precise the "conformally reasonable behavior" mentioned above. We work out two examples in detail: the conformal Laplacian $Y=\Delta+\tau / 6$ ( $\tau=$ scalar curvature) with Dirichlet conditions, and $Y$ with conditions of Neumann type called Robin conditions by physicists; specifically, the boundary operator here is $N-H / 3$, where $N$ is the inward unit normal derivative, and $H$ is the trace of the boundary embedding's second fundamental form.

This paper is organized as follows. In $\S 1$, we summarize the invarianttheoretic background needed to extract information from the heat asymptotics on manifolds with boundary. Section 1 also describes a natural fourth-order differential operator $P$, originally introduced by Paneitz [P] (see also $[\mathrm{Br} 2$, ES]) in connection with the interaction of the gauge and conformal groups on Maxwell fields; $P$ seems to be absolutely central to four-dimensional functional determinant problems. In $\S 1$, we also make precise statements of the abovementioned analytic and naturality assumptions. In $\S 2$, we define the functional 
determinant and prove a formula of Polyakov type for its conformal variation. (See also [R, BØ2] in the boundariless case.) Though the functional determinant is a nonlocal invariant of the spectrum of $(A, B)$ (i.e., it is not the integral of a local expression), its conformal variation is local, and in fact is a heat invariant. In $\S \S 3$ and 4 , we apply this variational formula in concert with invariant-theoretic and conformal geometric knowledge of the heat invariants in dimension four to get explicit local formulas for the quotient of functional determinants. At this point, the operators $A$ and $B$ have not been pinned down, apart from their naturality and conformal behavior; thus our formulas at this point depend on (exactly 13) parameters. In $\S 6$ we compute these parameters for the two choices of $(A, B)$ mentioned above: the conformal Laplacian with Dirichlet and Robin conditions. In $\S 5$, still in the abstract (parameterdependent) setting, we compute determinant quotients on special manifolds; specifically the unit four-hemisphere $H^{4}$ and the cylinder $\mathscr{C}_{h}^{4}=[0, h] \times S^{3}$ for $h>0$, both with their standard metrics. Since $H^{4}$ is conformally equivalent to the unit ball $B^{4}$, and $\mathscr{C}_{h}^{4}$ is conformally equivalent to the spherical shell $\mathscr{A}_{s}^{4}=\left\{x \in \mathbb{R}^{m}|1 \leq| x \mid \leq s\right\}$ when $s=e^{h}$, we get as special cases determinant quotients for the pairs $\left(B^{4}, H^{4}\right)$ and $\left(\mathscr{A}_{s}^{4} \mathscr{C}_{h}^{4}\right)$. Since everything is explicitly computable on $H^{4}(\S 7)$, this means that we get an exact value for the functional determinant on $B^{4}$. For example, we find that if $Y_{-}$(resp. $Y_{+}$) is the conformal Laplacian with Dirichlet (resp. Robin) conditions, then

$$
\begin{aligned}
-\log \operatorname{det} Y_{ \pm}= & \frac{1}{3} \zeta_{R}^{\prime}(-3)+\frac{1}{6} \zeta_{R}^{\prime}(-1)+\frac{1}{288} \pm \frac{1}{2} \zeta_{R}^{\prime}(-2) \text { on } H^{4}, \\
-\log \operatorname{det} Y_{-}= & \frac{1}{3} \zeta_{R}^{\prime}(-3)+\frac{1}{6} \zeta_{R}^{\prime}(-1) \\
& +\frac{1}{288}-\frac{1}{2} \zeta_{R}^{\prime}(-2)+\left(4 \log 2+\frac{17}{21}\right) / 360 \text { on } B^{4}, \\
-\log \operatorname{det} Y_{+}= & \frac{1}{3} \zeta_{R}^{\prime}(-3)+\frac{1}{6} \zeta_{R}^{\prime}(-1) \\
& +\frac{1}{288}+\frac{1}{2} \zeta_{R}^{\prime}(-2)+\left(4 \log 2-\frac{1}{3}\right) / 360 \text { on } B^{4},
\end{aligned}
$$

where $\zeta_{R}$ is the Riemann zeta function. As a consequence (Corollary 6.7), we find that passage from the round $H^{4}$ metric to the flat $B^{4}$ metric "improves" the appropriate scale-invariant version of either functional determinant, so that the extremal result of [BCY, §5] for $\operatorname{det} Y$ on $S^{4}$ will not readily extend to the hemisphere. In an appendix $(\S 8)$, we collect in one place the definitions of local invariants used in developing the determinant quotient formulas, and prove some facts (used in $\S 7$ ) about zeta functions associated to spheres.

Special thanks are due to Bent Ørsted for enlightening discussions.

\section{LOCAL INVARIANTS, NATURAL DIFFERENTIAL OPERATORS AND BOUNDARY VALUE PROBLEMS, AND THE HEAT INVARIANTS}

Let $M$ be a smooth, compact, $m$-dimensional Riemannian manifold with smooth boundary $\partial M$. Denote by $g$ the metric tensor on $M$; the pullback of $g$ under the inclusion $\partial M \hookrightarrow M$ is a Riemannian metric on $\partial M$. Let $R$ be the Riemann curvature tensor of $g$, with the sign convention that makes $R^{1}{ }_{212}$ 
positive on standard spheres. We adopt the convention that letters $i, j, \ldots$ run from 1 to $m$, and index a local coordinate frame and coframe on $M$. We raise and lower indices using the metric tensor, and sum over repeated indices. The Ricci tensor $\rho$ of $M$ has $\rho_{i j}=R^{k}{ }_{i k j}$, and the scalar curvature of $M$ is $\tau=\rho^{i}{ }_{i}$.

Additional invariants describe the embedding of $\partial M$, and are defined as tensor fields over $\partial M$ (as opposed to $M$ ). Let $N$ be the inward unit geodesic normal in a collar for $\partial M$ in $M$, and consider local coordinates $\left(x^{i}\right)$ in a neighborhood of a point of $\partial M$ for which $\partial / \partial x^{m}=N$, and for which the $x^{a}, a=1, \ldots, m-1$ are local coordinates on $\partial M$. Letters $a, b, \ldots$ will run from 1 to $m-1$, and index coordinate frames and coframes of this type on $\partial M$. The subscript $N$ will be interchangeable with $m$ in this setting, and will serve to indicate that we are working in such a coordinate system. We denote the coordinate coframe element $d x^{m}$ by $N_{b}$. The (second) fundamental form $L$ of the boundary embedding is a symmetric 2 -tensor defined by

$$
L_{a b}:=-\frac{1}{2} N g_{a b} \text {. }
$$

The trace $H:=L^{a}{ }_{a}$ of $L$ is a multiple of the mean curvature. Here we have used $\left.g\right|_{\partial M}$, the pullback of $g$ to $\partial M$ under the inclusion, to raise the boundary index; we shall always use $\left.g\right|_{\partial M}$ as the metric on $\partial M$. Repeated boundary indices are, of course, summed from 1 to $m-1$. $L$ measures the deviation of the boundary embedding from total geodesy; that is, it is the obstruction to the possibility of finding coordinates $x^{i}$ which are normal on both $M$ and $\partial M$.

A symmetric 2-tensor $G$ is defined by

$$
G_{b}^{a}:=R^{a}{ }_{N b N},
$$

and we let $F:=G^{a}{ }_{a}$. The symmetric 2-tensor $T$ is defined by

$$
T_{a b}:=R_{a c b}^{c} \text {. }
$$

Note that $(T+G)_{a b}=\rho_{a b}$, and that $T^{a}{ }_{a}=R^{c a}{ }_{c a}=\tau-2 F$. We use $g$ and its pullback $\left.g\right|_{\partial M}$ to define quantities like $|\rho|^{2}=\rho^{i j} \rho_{i j},|L|^{2}=L^{a b} L_{a b},\langle L, G\rangle=$ $L^{a b} G_{a b}$, etc. Intrinsic objects on $\partial M$ which are analogous to objects on $M$ will usually be denoted with a tilde; for example, $\tilde{g}=\left.g\right|_{\partial M} ; \nabla, \tilde{\nabla}$ are the LeviCivita connections on $M$ and $\partial M$ respectively; and $\Delta, \tilde{\Delta}$ are the Laplacians on functions. The Riemannian measure on $(M, g)$ will be denoted by $d x$, and the Riemannian measure on $(\partial M, \tilde{g})$ by $d y$. Our sign convention for the Laplacian gives $\Delta=-d^{2} / d x^{2}$ on $\mathbb{R}^{1}$. We shall sometimes use a standard abbreviation in which indices after a bar indicate covariant differentiations with respect to $\nabla$, for example $\varphi_{i j \mid k l}=\nabla_{l} \nabla_{k} \varphi_{i j}:=(\nabla \nabla \varphi)_{l k i j}$; and indices after a colon similarly indicate covariant differentiations with respect to $\tilde{\nabla}$.

Let

$$
\begin{aligned}
J & =\tau / 2(m-1), \quad V=(\rho-J g) /(m-2), \\
C^{i}{ }_{j k l} & =R^{i}{ }_{j k l}+V_{j k} \delta^{i}{ }_{l}-V_{j l} \delta^{i}{ }_{k}+V^{i}{ }_{l} g_{j k}-V^{i}{ }_{k} g_{j l} .
\end{aligned}
$$

$C$ is the Weyl conformal curvature tensor. $C, V, J$ carry the information in $R$ in a way which is better adapted to conformal variational calculations than are $R, \rho, \tau$. Specifically, let the metric run through a conformal curve $g[\varepsilon \omega]=$ 
$e^{2 \varepsilon \omega} g[0]$ for $\omega \in C^{\infty}(M)$ and $\varepsilon$ a real parameter. Then $\left.(d / d \varepsilon)\right|_{\varepsilon=0}(g[\varepsilon \omega])=$ $2 \omega g[0]$ and

$$
\begin{aligned}
\left.(d / d \varepsilon)\right|_{\varepsilon=0} C[\varepsilon \omega] & =0, \\
\left.(d / d \varepsilon)\right|_{\varepsilon=0} J[\varepsilon \omega]+2 \omega J[0] & =\Delta[0] \omega, \\
\left.(d / d \varepsilon)\right|_{\varepsilon=0} V[\varepsilon \omega] & =-(\nabla \nabla)[0] \omega .
\end{aligned}
$$

Here we have used the following convention, which will be maintained throughout this paper: given a conformal class of metrics

$$
\langle g[0]\rangle:=\left\{e^{2 \omega} g[0] \mid \omega \in C^{\infty}(M)\right\},
$$

and a metric-dependent quantity $T$, we indicate that $T$ should be evaluated in $g[\omega]:=e^{2 \omega} g[0]$ by writing $T[\omega]$. For example, the conformal invariance of the density $|C|^{2} d x$ on four-manifolds can be expressed as

$$
\left(|C|^{2} d x\right)[\omega]=\left(|C|^{2} d x\right)[0], \quad m=4, \omega \in C^{\infty}(M) .
$$

We shall need the Paneitz quantity

$$
Q=-2|V|^{2}+m J^{2} / 2+\Delta J
$$

and Paneitz operator

$$
P=\Delta^{2}+\delta\{(m-2) J-4 V \cdot\} d+(m-4) Q / 2 .
$$

Here $d$ is the exterior derivative, $\delta$ is the formal adjoint of $d$, and $V$. is the bundle endomorphism $\varphi=\left(\varphi_{i}\right) \mapsto\left(V_{i}^{j} \varphi_{j}\right)$ on the cotangent bundle $T^{*} M$. $\mathrm{By}[\mathrm{P} ; \mathrm{Br} 2$, Theorem $1.21 ; \mathrm{ES}], P$ is conformally covariant: given a conformal class $\left\langle g_{0}\right\rangle$,

$$
e^{(m+4) \omega / 2} P[\omega]=P[0] \mu\left(e^{(m-4) \omega / 2}\right), \quad \text { all } \omega \in C^{\infty}(M), \quad m \neq 1,2,
$$

where for any $u \in C^{\infty}(M), \mu(u)$ denotes multiplication by $u$. The infinitesimal form of $(1.6)$ is

$$
\left.(d / d \varepsilon)\right|_{\varepsilon=0} P[\varepsilon \omega]=-4 \omega P[0]+\frac{m-4}{2}[P[0], \mu(\omega)] .
$$

A conformal variational formula for the local scalar invariant $Q$ in dimension $m=4$ will be important for us. To get this, let $m \geq 3$ be arbitrary for the moment, and let

$$
P_{0}=P-\frac{m-4}{2} Q=\Delta^{2}+\delta\{(m-2) J-4 V \cdot\} d .
$$

Applying the conformal covariance relation (1.6) to the function 1, we get

$$
\begin{aligned}
\frac{m-4}{2} Q[\omega] e^{(m+4) \omega / 2} & =\left(P_{0}[0]+\frac{m-4}{2} Q[0]\right) e^{(m-4) \omega / 2} \\
& =P_{0}[0]\left(e^{(m-4) \omega / 2}-1\right)+\frac{m-4}{2} Q[0] e^{(m-4) \omega / 2},
\end{aligned}
$$

since $P_{0}$ annihilates constants. This leads to the identity

$$
\begin{aligned}
Q[\omega] e^{(m+4) \omega / 2} & =\frac{2}{m-4} P_{0}[0]\left(e^{(m-4) \omega / 2}-1\right)+Q[0] e^{(m-4) \omega / 2} \\
& =P[0]\left(\omega+(m-4) \omega^{2} a((m-4) \omega)\right)+Q[0]
\end{aligned}
$$


where $a$ is an entire function. This identity holds for $m \neq 4$, but since all conformal variational calculations can be done within spaces of polynomial invariants with rational-in- $m$ coefficients, analytic continuation in $m$ is justified, and we get

$$
P[0] \omega+Q[0]=Q[\omega] e^{4 \omega}, \quad m=4 .
$$

Taking the variation of (1.7), we have

$$
P[0] \omega=\left.(d / d \varepsilon)\right|_{\varepsilon=0} Q[\varepsilon \omega]+4 \omega Q[0], \quad m=4 .
$$

1.1. Remark. We shall work with differential operators on bundles of tensorspinors over $(M, g)$. One way to describe these is as follows. A tensor-spinor bundle $\mathbb{V}$ is a vector bundle associated to the principal $\mathrm{O}(m)$-bundle of orthonormal frames, the $\mathrm{SO}(m)$-bundle of oriented orthonormal frames, or the $\operatorname{Spin}(m)$-bundle of spin frames. (The groups $H=\mathrm{O}(m), \mathrm{SO}(m)$, and $\operatorname{Spin}(m)$ are the natural structure groups of Riemannian, oriented Riemannian, and Riemannian spin geometry respectively.) That is, $\mathbb{V}$ has the form $\mathscr{F}_{H} \times_{\rho} V$, where $(V, \rho)$ is a finite-dimensional representation of $H$, and $\mathscr{F}_{H}$ is the appropriate frame bundle. Since the defining representation $T$ of $\mathrm{SO}(m)$ and the spin representation $\Sigma$ of $\operatorname{Spin}(m)$ are faithful, any irreducible tensor-spinor bundle can be realized as a direct summand of an iterated tensor product

$$
\mathscr{G}_{\beta, \tau}^{\alpha, \sigma}=(T M)^{\otimes \alpha} \otimes\left(T^{*} M\right)^{\otimes \beta} \otimes(\Sigma M)^{\otimes \sigma} \otimes\left(\Sigma^{*} M\right)^{\otimes \tau}, \quad \alpha, \beta, \sigma, \tau \in \mathbb{N} .
$$

We shall need a quick review of some basic results on the small time asymptotic expansion of the trace of the heat operator. Details can be found in [G2], especially $\S 1.9$.

1.2. Analytic assumptions. Let $A$ be a differential operator of positive order on sections of a tensor-spinor bundle $\mathbb{V}$ over $M$. Suppose that $A$ is formally selfadjoint and has positive definite leading symbol $\sigma_{\text {lead }}(A)$; that is, $\sigma_{\text {lead }}(A)(x, \xi)$ is positive definite in End $\mathbb{V}_{x}$ for all $x \in M$ and $0 \neq \xi \in T_{x}^{*} M$. Let $B$ be an operator on the bundle of Cauchy data for $A$ on $\partial M$ with the property that the pair $(A, B)$ is elliptic.

1.3. Remark. Formal selfadjointness and the assumption on the leading symbol make sense because tensor-spinor bundles over a Riemannian manifold come equipped with Riemannian vector bundle structures. Since $\sigma_{\text {lead }}(A)(x,-\xi)$ $=(-1)^{\operatorname{ord}(A)} \sigma_{\text {lead }}(A)(x, \xi)$, the assumption of positive definite leading symbol forces the order of $A$ to be even. We shall always denote $\operatorname{ord}(A)$ by $2 l>0$, so that $\sigma_{\text {lead }}(A)=\sigma_{2 l}(A)$. We do not give the definition of ellipticity for a boundary value problem here as it is somewhat technical and distracting; see [G2, §1.9] for this.

1.4. Remark. The bundle $W$ of order $2 l$ Cauchy data for sections of $\mathbb{V}$ has a natural grading by subbundles

$$
W=W_{0} \oplus \cdots \oplus W_{2 l-1}
$$

where $W_{j}$ holds the $j$ th Cauchy datum. The boundary operator $B$ for an elliptic boundary value problem is valued in an auxiliary bundle $W^{\prime}$ which admits a similar grading

$$
W^{\prime}=W_{0}^{\prime} \oplus \cdots \oplus W_{2 l-1}^{\prime}
$$


but which has $\operatorname{dim} W^{\prime}=\frac{1}{2} \operatorname{dim} W$. (See the examples below.)

Let $A_{B}$ be the restriction of $A$ to the subspace

$$
C^{\infty}(M, \mathbb{V})_{B}=\left\{F \in C^{\infty}(M, \mathbb{V}) \mid B\left(\mathrm{CD}_{2 l} F\right)=0\right\} .
$$

Here $\mathrm{CD}_{2 l}: C^{\infty}(M, \mathbb{V}) \rightarrow C^{\infty}(\partial M, W)$ is the operator which assigns the order $2 l$ Cauchy data. If $f \in C^{\infty}(M)$, there is an asymptotic expansion

$$
\operatorname{Tr}_{L^{2}} f \exp \left(-t A_{B}\right) \sim \sum_{n=0}^{\infty} a_{n}(f, A, B) t^{(n-m) / 2 l}, \quad t \downarrow 0,
$$

where

$$
a_{n}(f, A, B)=\int_{M} f a_{n}(x, A) d x+\sum_{\nu=0}^{n-1} \int_{\partial M}\left(N^{\nu} f\right) a_{n, \nu}(y, A, B) d y .
$$

The $a_{n}(x, A)$ and $a_{n, \nu}(y, A, B)$ are locally computable from the total symbols of $A$ and $B$ in local coordinates.

1.5. Remark. The auxiliary function $f$ is a device which allows us to observe the distributional behavior of the heat kernel at the boundary. We are forced to deal with this extra information because, as we shall see below, conformal deformation of the asymptotics of $\operatorname{Tr} \exp \left(-t A_{B}\right)$ and of the functional determinant naturally lead to the asymptotics of $\operatorname{Tr} \omega \exp \left(-t A_{B}\right)$, where $\omega$ is the infinitesimal conformal factor as above. Here and below, we write simply "Tr" for $\operatorname{Tr}_{L^{2}}$, and use the notation "tr" for traces over vector bundle fibers.

1.6. Naturality assumptions. Suppose that $A$ and $B$ are given locally by universal, polynomial formulas in the jets of a Riemannian metric $g$; the inverse $g^{\sharp}$ of $g$; plus (if orientation is involved), a volume form $E$; plus (if spin structure is involved) the fundamental tensor-spinor $\gamma$. Suppose that, with respect to uniform dilations of the metric, $A$ has homogeneity degree - ord $A$, and the boundary condition does not change: if $0<\alpha \in \mathbb{R}$,

$$
\begin{gathered}
\bar{g}=\alpha^{2} g \quad\left(\bar{E}=\alpha^{m} E, \bar{\gamma}=\alpha^{-1} \gamma\right) \Rightarrow \bar{A}=\alpha^{-2 l} A, \\
\mathcal{N}\left(\bar{B} \circ \overline{\mathrm{CD}}_{2 l}\right)=\mathscr{N}\left(B \circ \mathrm{CD}_{2 l}\right),
\end{gathered}
$$

where $\mathscr{N}$ is the null space. Suppose further that $A$ satisfies the analytic assumptions 1.2 categorically; that is, the realization of $(A, B)$ on any Riemannian manifold $(M, \partial M)$ with boundary satisfies the analytic assumptions.

1.7. Remark. If $M$ has spin structure, the fundamental tensor-spinor, or Clifford section $\gamma$ is a section of $T M \otimes \operatorname{End} \Sigma M \cong \operatorname{Spin}(m) T M \otimes \Sigma M \otimes \Sigma^{*} M$, where $T M$ is the tangent bundle and $\Sigma M$ the spinor bundle. $\gamma$ satisfies the Clifford relations

$$
\gamma^{i} \gamma^{j}+\gamma^{j} \gamma^{i}=-2 g^{i j} \mathrm{Id}_{\Sigma}
$$

where $\gamma^{i}$ is the local section of End $\Sigma$ gotten by tensoring with $d x^{i}$ and contracting the $T M$ argument. The scalings of $E$ and $\gamma$ posited in (1.10) are those which are consistent with the scaling of the metric; the scaling of $\gamma$ being forced by the Clifford relations. The Levi-Civita connection on $T M$ is lifted to the spinor bundle (if any), and extended to iterated tensor products of $T M$, $\Sigma M$, and their duals, so that we may take covariant derivatives of tensor-spinor fields. $\nabla g, \nabla E$, and $\nabla \gamma$ all vanish when defined. 
1.8. Remark. Since a scaling $\bar{g}=\alpha^{2} g$ of the metric induces a scaling $\bar{N}=$ $\alpha^{-1} N$ of the inward unit normal, the operator $\mathrm{CD}_{2 l}$ is sensitive to uniform dilation; thus we had to speak of $\overline{\mathrm{CD}}_{2 l}$ in (1.10).

1.9. Remark. By Weyl's invariant theory, $A$ is built polynomially (using tensor product and contraction) from $g$, its inverse $g^{\sharp}, \nabla$, and iterated covariant derivatives of $R$; plus (if orientation is involved) $E$; plus (if spin structure is involved) $\gamma . B$ is similarly built from $\tilde{g}, \tilde{g}^{\sharp}, N, N_{b}, \nabla_{N}, \tilde{\nabla}$, and tangential covariant derivatives of $R$ and $L$, plus the restrictions to $\partial M$ of $E$ and/or $\gamma$ if applicable. As a result, the $a_{n}(x, A)$ are polynomial in $g, g^{\sharp}$, and iterated covariant derivatives of $R$; plus $E$ and/or $\gamma$ if applicable. The $a_{n, \nu}(y, A, B)$ are polynomial in $\tilde{g}, \tilde{g}^{\sharp}, N, N_{b}$, and iterated tangential covariant derivatives $(\tilde{\nabla})$ of $R_{\partial M}$ and of $L$, plus the restriction to $\partial M$ of $E$ and/or $\gamma$ if applicable.

1.10. Remark. We shall say that a local scalar invariant on $M$ or $\partial M$, or a natural differential operator $\mathscr{A}$ on some $C^{\infty}(M, \mathbb{V})$, has level $n$ if it scales according to $\overline{\mathscr{A}}=\alpha^{-n} \mathscr{A}$ under uniform dilation $\bar{g}=\alpha^{2} g$ of the metric, $0<\alpha \in \mathbb{R}$ (with the compatible scalings $\bar{E}=\alpha^{m} E, \bar{\gamma}=\alpha^{-1} \gamma$ if applicable). For example, it is part of the naturality assumptions 1.6 that $A$ has level $2 l$ (equal to its order). It is straightforward to show that we may measure the level as follows. If $\mathscr{A}$ is a level $n$ monomial local invariant or monomial natural differential operator on $M$, of degree $\left(k_{R}, k_{\nabla}\right)$ in $(R, \nabla)$, then $2 k_{R}+k_{\nabla}=n$. If $\mathscr{A}$ is a level $n$ monomial local invariant or monomial natural differential operator on $\partial M$, of degree $\left(k_{R}, k_{L}, k_{\tilde{\nabla}}, k_{N}\right)$ in $\left(R, L, \tilde{\nabla}, \nabla_{N}\right)$, then

$$
2 k_{R}+k_{L}+k_{\tilde{\nabla}}+k_{N}=n .
$$

In the study of the index, analytic torsion, and functional determinant, a special role is played by quantities of level $m$, the dimension. Thus in this paper, we shall be especially interested in level 4 objects on $M$, for example the Paneitz quantity $Q$ and operator $P$; and level 3 objects on $\partial M$.

1.11. Remark. By the last two remarks, the assumption of categorically positive definite leading symbol implies that $\sigma_{2 l}(A)$ is polynomial in $g$ and $g^{\sharp}$; plus, if applicable, $E$ and/or $\gamma$; that is, no higher jets of these objects are involved.

1.12. Remark. Parity considerations force $a_{\text {odd }}(x, A)=0$, but the $a_{\text {odd }, \nu}(y, A, B)$ are generally nonzero. Homogeneity considerations (i.e., comparison of the behavior of the two sides of (1.9) under uniform dilation of the metric) imply that $a_{n}(x, A)$ has level $n$. Similarly, $a_{n, \nu}(y, A, B)$ must have level $n-1-\nu$.

\section{THE FUNCTIONAL DETERMINANT AND ITS CONFORMAL VARIATION}

We retain the notation of $\S 1$, and assume that our boundary value problem $(A, B)$ satisfies the analytic and naturality assumptions $1.2,1.6$. The analytic assumptions guarantee that $(A, B)$ has real eigenvalue spectrum $\lambda_{0} \leq \lambda_{1} \leq$ $\ldots \uparrow+\infty$, with corresponding eigensections in $C^{\infty}(M, \mathbb{V})_{B}$. We define the zeta function of the problem $(A, B)$ by

$$
\zeta_{A, B}(s)=\sum_{\lambda_{j} \neq 0}\left|\lambda_{j}\right|^{-s}
$$


There exist $\varepsilon>0$ and $j_{0} \in \mathbb{N}$ such that $\lambda_{j} \geq j^{\varepsilon}$ whenever $j \geq j_{0}$, so $\zeta_{A, B}(s)$ is manifestly well-defined and holomorphic for large $\operatorname{Re} s$. Since there are only finitely many nonpositive $\lambda_{j}$, the heat expansion (1.8) gives

$$
\begin{aligned}
\sum_{\lambda_{j} \neq 0} e^{-t\left|\lambda_{j}\right|}= & -q(A, B)+2 \sum_{\lambda_{j}<0} \sinh t \lambda_{j} \\
& +\sum_{n=0}^{N} a_{n}(A, B) t^{(n-m) / 2 l}+O\left(t^{(N-m+1) / 2 l}\right) \\
= & \sum_{n=0}^{N} \underline{a}_{n}(A, B) t^{(n-m) / 2 l}+O\left(t^{(N-m+1) / 2 l}\right),
\end{aligned}
$$

where $a_{n}(A, B)=a_{n}(1, A, B), q(A, B)$ is the multiplicity of 0 as an eigenvalue of $(A, B)$, and

$$
\underline{a_{n}(A, B)}= \begin{cases}a_{n}(A, B)-q(A, B), & n=m, \\ a_{n}(A, B)+2 \sum_{\lambda_{j}<0} \frac{\lambda_{j}^{k}}{k !}, & n=m+2 l(1+2 k), k \in \mathbb{N}, \\ a_{n}(A, B) & \text { otherwise. }\end{cases}
$$

Applying the Mellin transform, we get a meromorphic continuation of $\zeta_{A, B}(s)$ to $\mathbb{C}$ :

$$
\begin{aligned}
\zeta_{A, B}(s)=\frac{1}{\Gamma(s)}( & \sum_{n=0}^{N}\left(s-\frac{m-n}{2 l}\right)^{-1} \underline{a_{n}(A, B)} \\
& \left.+\int_{0}^{1} t^{s-1} O\left(t^{(N-m+1) / 2 l}\right) d t+\int_{1}^{\infty} t^{s-1} \sum_{\lambda_{j} \neq 0} e^{-t\left|\lambda_{j}\right|} d t\right),
\end{aligned}
$$

where $O\left(t^{(N-m+1) / 2 l}\right)$ is the error term from (2.1). In particular, $\zeta_{A, B}(s)$ is regular at $s=0$, and we define the functional determinant of the problem $(A, B)$ by

$$
\operatorname{det} A_{B}=(-1)^{\#\left\{\lambda_{j}<0\right\}} \exp \left(-\zeta_{A, B}^{\prime}(0)\right) .
$$

2.1. Remark. It is important to note that the functional determinant is not invariant under uniform dilation of the metric. Suppose, as before, that $\bar{g}=$ $\alpha^{2} g$, and if applicable, $\bar{E}=\alpha^{m} E, \bar{\gamma}=\alpha^{-1} \gamma$. Then

$$
\zeta_{\bar{A}, \bar{B}}(0)=\zeta_{A, B}(0), \quad \operatorname{det} \overline{A_{B}}=\alpha^{-2 l \zeta_{A, B}(0)} \operatorname{det} A_{B} .
$$

That is, the quantity $\zeta_{A, B}(0)$ is scale-invariant, and the functional determinant has a scale homogeneity which depends on $\zeta_{A, B}(0)$. Thus the functional

$$
\mathscr{P}(A, B, g)=\operatorname{vol}(g)^{2 / \zeta_{A, B}(0) / m} \operatorname{det} A_{B}
$$

is a scale-invariant "version" of the determinant. An added advantage of $\mathscr{P}(A, B, g)$ is that, like the determinant, it is a spectral invariant, since $a_{0}(A, B)=C \operatorname{vol}(g)$, where $C$ is a constant depending only on $\sigma_{2 l}(A)$. (The number $2 l / m$ can be recovered from the spectrum because $-m / 2 l$ is the leading exponent in the heat asymptotics (1.8).) We emphasize that there is no 
reason to expect $\operatorname{det} A_{B}$ or $\mathscr{P}(A, B, g)$ to be the integral of a local expression, as is $a_{n}(A, B)$.

\subsection{Remark. If $m>1$ and $\partial M \neq \varnothing$, the functional}

$$
\mathscr{P}_{\lambda}(A, B, g)=\operatorname{vol}(g)^{2 l \lambda / m} \operatorname{vol}(\tilde{g})^{2 l\left(\zeta_{A, B}(0)-\lambda\right) /(m-1)} \operatorname{det} A_{B}, \quad \lambda \in \mathbb{R},
$$

is also scale-invariant, and this raises the interesting prospect of interaction with the isoperimetric functional, especially in connection with extremal problems. The new ingredient, $\operatorname{vol}(\tilde{g})$, is often a spectral invariant: $a_{1}(A, B)$ has the form $C \operatorname{vol}(\tilde{g})$ for some constant $C$ which depends on $(A, B)$ but not on $M$. Thus $\operatorname{vol}(\tilde{g})$ is determined by the spectrum when $C \neq 0$. To preserve the spirit of the endeavor, and with a view toward the isospectral problem, one would like to choose exponents in (2.4) which are spectral invariants, perhaps by choosing $\lambda=0$ or $\lambda=\zeta_{A, B}(0)$. (See Theorem 4.10.)

2.3. Remark. Suppose we are given an elliptic boundary value problem $(D, b)$ in which $D$ is formally selfadjoint, but does not necessarily have positive definite leading symbol. Let $d$ be the order of $D$. if $r \in \mathbb{Z}^{+}$, we can form a new elliptic problem $\left(D^{r}, b^{(r)}\right)$ by taking the $r$ th power: the boundary condition determining $b^{(r)}$ is

$$
b \circ \mathrm{CD}_{d} \varphi=b \circ \mathrm{CD}_{d}(D \varphi)=\cdots=b \circ \mathrm{CD}_{d}\left(D^{r-1} \varphi\right)=0 \text {. }
$$

The operator corresponding to the problem $\left(D^{r}, b^{(r)}\right)$ will be called $\left(D_{b}\right)^{r}$. If $(D, b)$ is natural, then so is $\left(D^{r}, b^{(r)}\right)$, and if $r$ is even, $D^{r}$ has positive definite leading symbol.

We shall now impose some additional conformal assumptions.

2.4. Conformal assumptions. Suppose that $A$ is a positive integer power of a natural differential operator $D, A=D^{h}$, which is conformally covariant in the sense that given a conformal class $\langle g[0]\rangle$,

$$
e^{a+2 l / h} D[\omega]=D[0] \mu\left(e^{a \omega}\right), \quad \omega \in C^{\infty}(M),
$$

for some $a \in \mathbb{R}$. Here, in case orientation and/or spin structure are used, $E[\omega]:=e^{m \omega} E[0], \gamma[\omega]:=e^{-\omega} \gamma[0]$ for some $a \in \mathbb{R}$. Suppose that $B$ arises as $b^{(h)}$ as in Remark 2.3 , where $(D, b)$ is an elliptic boundary value problem, and that the conformal behavior of $b$ is compatible with (2.5) in the sense that $\mathscr{N}\left(\left(b \circ \mathrm{CD}_{2 l / h}\right)[\omega]\right)=\mathscr{N}\left(\left(b \circ \mathrm{CD}_{2 l / h}\right)[0] \mu\left(e^{a \omega}\right)\right)$, or equivalently,

$$
\mathscr{N}\left(\left(b \circ \mathrm{CD}_{2 l / h}\right)[\omega]\right)=e^{-a \omega} \mathscr{N}\left(\left(b \circ \mathrm{CD}_{2 l / h}\right)[0]\right)
$$

2.5. Remark. Our conformal assumptions are weaker than the assertion that $(A, B)$ is conformally covariant; this is the special case $h=1$. When we work in this generality, we can handle, for example, the conformal Laplacian $D$ on middle-forms ( $m / 2$-forms for $m$ even) with a suitable boundary operator $B$. By $[\mathrm{Br} 1], D$ has the form

$$
\delta d-d \delta+(\text { Ricci term }),
$$

where $d$ is the exterior derivative, and $\delta$ is the formal adjoint of $d$. If $M$ is oriented, $D$ interchanges the two eigenbundles $\Lambda_{ \pm}^{m / 2} M$ of the Hodge $\star$ operator, unlike the form Laplacian $\Delta=\delta d+d \delta$, which preserves both $\Lambda_{+}^{m / 2} M$ and $\Lambda_{-}^{m / 2} M$. There do, in fact exist boundary conditions which are suitable in 
the sense of ellipticity and conformal covariance of the right weight. Note that since

$$
D^{2}=\Delta^{2}+(\text { lower order }),
$$

$D$ is elliptic. On the other hand, the Dirichlet problem for the spin Laplacian, i.e., for the square of the Dirac operator $\nabla$ on the spinor bundle $\Sigma M$, is outside the framework we have described, even though $\nabla$ is conformally covariant. The reason is that Dirichlet boundary conditions for $\nabla^{2}$ do not arise from the iteration process of Remark 2.3. In fact, there are no local boundary conditions for $\nabla$ which are elliptic in the sense we need, though there are appropriate nonlocal (Atiyah-Patodi-Singer) conditions.

2.6. Remark. The infinitesimal form of the conformal covariance relation $(2.5)$ is

$$
\left.(d / d \varepsilon)\right|_{\varepsilon=0} D[\varepsilon \omega]=-(2 l \omega / h) D[0]+a[D[0], \mu(\omega)] .
$$

The finite and infinitesimal forms of the conformal covariance relation are, in fact, equivalent: an application of (2.7) with $g\left[\varepsilon_{0} \omega\right]$ in place of $g$ [0] gives

$$
\left.(d / d \varepsilon)\right|_{\varepsilon=\varepsilon_{0}}\left\{e^{(a+2 l / h) \varepsilon \omega} D[\varepsilon \omega] \mu\left(e^{-a \varepsilon \omega}\right)\right\}=0
$$

for any $\varepsilon_{0} \in \mathbb{R}$, so that $(2.5)$ is obtained. In practice, the way in which we shall enforce (2.6) is to show that

$$
\left(b \circ \mathrm{CD}_{2 l / h}\right)[\omega]=\mathscr{A}[\omega]\left(b \circ \mathrm{CD}_{2 l / h}\right)[0] \mu\left(e^{a \omega}\right) \text {, }
$$

where $\mathscr{A}[\omega]$ is a smooth, functorial, $\omega$-dependent section of Aut $W^{\prime}$, with the curves $\mathscr{A}[\varepsilon \omega]$ smooth and $\mathscr{E}[0]=I_{W^{\prime}}$. An argument like (2.8) shows that this is in turn enforced by its infinitesimal form

$$
\left.(d / d \varepsilon)\right|_{\varepsilon=0}\left(b \circ \mathrm{CD}_{2 l / h}\right)[\varepsilon \omega]=a\left(b \circ \mathrm{CD}_{2 l / h}\right)[0] \mu(\omega)+\mathscr{E}[\omega]\left(b \circ \mathrm{CD}_{2 l / h}\right)[0],
$$

where $\mathscr{E}[\omega]:=\left.(d / d \varepsilon)\right|_{\varepsilon=0} \mathscr{A}[\varepsilon \omega] \in C^{\infty}\left(\partial M\right.$, End $\left.W^{\prime}\right)$. In fact, in our examples, the entries of $\mathscr{A}[\omega]$ in the block decomposition corresponding to the grading of Remark 1.4 have the form $\mu\left(e^{c \omega}\right)$ for various powers $c$.

2.7. Example. Let $A$ be the conformal Laplacian, or Yamabe operator

$$
Y=\Delta+\frac{m-2}{4(m-1)} \tau \text {. }
$$

$Y$ is conformally covariant of bidegree $((m-2) / 2,(m+2) / 2)$ :

$$
Y[\omega]=e^{-(m+2) \omega / 2} Y[0] \mu\left(e^{(m-2) \omega / 2}\right) .
$$

Though $Y$ can be viewed as a conformally invariant operator between density bundles, we choose not to do so, and instead view it as acting on sections of a trivial line bundle over $M$. Accordingly, Dirichlet conditions for $Y$ are obtained by letting $W_{0}$ be a trivial line bundle over $\partial M$, setting $W_{1}=0$, and setting

$$
B_{0,0}=\text { Id }, \quad B_{0,1}=B_{1,0}=B_{1,1}=0
$$

in the block decomposition of Remark 1.4. Dirichlet conditions are, of course, conformally compatible.

2.8. Example. There is also a conformally compatible Neumann condition, sometimes called the Robin condition by physicists. This obtained by "playing 
off" the conformal variation of the mean curvature against that of the normal vector field $N$, just as the variation of the scalar curvature $\tau$ compensates that of the Laplacian $\Delta$ to form the conformal Laplacian. By [BG, Appendix],

$$
\left.(d / d \varepsilon)\right|_{\varepsilon=0} N[\varepsilon \omega]=-\omega N[0],\left.\quad(d / d \varepsilon)\right|_{\varepsilon=0} H[\omega]+\omega H[0]=-(m-1) \omega_{\mid N} .
$$

Thus for all $a, \alpha \in \mathbb{R}$,

$$
\begin{aligned}
& \left.(d / d \varepsilon)\right|_{\varepsilon=0}(N+a H)[\varepsilon \omega]+\omega(N+a H)[0]-\alpha[(N+a H)[0], \mu(\omega)] \\
& \quad=(-a(m-1)-\alpha) \mu\left(\omega_{\mid N}\right) .
\end{aligned}
$$

As a result, there is an infinitesimal conformal covariance law for $\nabla+a H$ for each $a \in \mathbb{R}$ :

$$
\left.(d / d \varepsilon)\right|_{\varepsilon=0}(N+a H)[\varepsilon \omega]=-\omega(N+a H)[0]-a(m-1)[(N+a H)[0], \mu(\omega)] .
$$

In particular, the boundary operator

$$
N-\frac{m-2}{2(m-1)} H
$$

is conformally compatible with $Y$. More precisely, to set up the Robin condition, we let $W_{1}^{\prime}$ be a trivial line bundle, $W_{0}^{\prime}=0, B_{1,1}=\mathrm{Id}, B_{0,1}=$ $-(m-2) H / 2(m-1)$, and $B_{0,0}=B_{1,0}=0$. The Robin condition is important in the study of the Yamabe problem on manifolds with boundary; see [E2].

2.9. Remark. If $(A, B)$ satisfies $1.2,1.6$, and 2.4 , then so does $\left(A^{r}, B^{(r)}\right)$ for each $r \in \mathbb{Z}^{+}$. This is sometimes useful in that it allows us to get rid of the (finite multiplicity) negative spectrum of $\left(A_{B}\right)$ by passing to $\left(A_{B}\right)^{2}$.

An extremely important property from our point of view is a generalization of the scale-invariance property (2.3) to pointwise (conformal) scalings under the conformal assumptions 2.4. Following [BØ1], we call this a conformal index property.

2.10. Conformal Index Theorem. If $(A, B)$ satisfies $1.2,1.6$, and 2.4 , and $g[0]$ is a Riemannian metric on $M$, then the quantities $q(A, B), \#\left\{\lambda_{j}<0\right\}$, $a_{m}(A, B)$, and $\zeta_{A, B}(0)$ are constant on the conformal class $\langle g[0]\rangle$.

Proof. Let $D$ be as in 2.4. The spectral invariants of $A[\omega]$ on $\mathscr{N}\left(\left(B \circ \mathrm{CD}_{2 l}\right)[\omega]\right)$ are the same as those of $\left(\mu\left(e^{-(a+2 l / h) \omega}\right) D[0] \mu\left(e^{a \omega}\right)\right)^{h}$ on $\mathscr{N}\left(\left(B \circ \mathrm{CD}_{2 l}\right)[0] \mu\left(e^{a \omega}\right)\right)$. The spectral invariants of the latter problem are the same as those of

$$
\underline{A[\omega]}:=e^{a \omega}\left(\mu\left(e^{-(a+2 l / h) \omega}\right) D[0] \mu\left(e^{a \omega}\right)\right)^{h} \mu\left(e^{-a \omega}\right)=\left(\mu\left(e^{-2 l \omega / h}\right) D[0]\right)^{h}
$$

on $\mathscr{N}\left(\left(B \circ \mathrm{CD}_{2 l}\right)[0]\right)$. Here we have applied a "global gauge transformation" in conjugating by $\mu\left(e^{a \omega}\right)$; this does not affect spectral data, and has the advantage of transforming the original problem into one in which the boundary condition is fixed. Note that all of the boundary value problems mentioned are elliptic because the original one is. Because $A_{B}$ has pure eigenvalue spectrum, the null spaces $\mathscr{N}(A)$ and $\mathscr{N}(D)$ in $C^{\infty}(M, \mathbb{V})_{B}$ agree. But by the conformal covariance relations, the dimension of $\mathscr{N}(D)$ in $C^{\infty}(M, \mathbb{V})_{B}$ is conformally invariant; thus $q(A, B)$ is conformally invariant. By a straightforward extension of an argument in [B1, Proposition 1], the number of negative 
eigenvalues of $\left(\mu\left(e^{-2 l \varepsilon \omega / h}\right) D[0]\right)^{h}$ on $\mathscr{N}\left(\left(B \circ \mathrm{CD}_{2 l}\right)[0]\right)$ is independent of $\varepsilon$; this uses the fact that the number of zero eigenvalues is independent of $\varepsilon$. Since $\zeta_{A, B}(0)=a_{m}(A, B)-q(A, B)$, we just need to show that $a_{m}(A, B)$ is conformally invariant.

For this, fix $\omega \in C^{\infty}(M)$, and consider the conformal curve of metrics $g[\varepsilon \omega]=e^{2 \varepsilon \omega} g[0]$. If we can show that the variation operator $\left.(d / d \varepsilon)\right|_{\varepsilon=0}$ annihilates the functional $a_{m}(A, B)$, we are done, since this result may then be applied with any $g\left[\varepsilon_{0} \omega\right]$ in place of $g[0]$, and $\omega$ is arbitrary. By the preceding paragraph, it is sufficient to show that $\left.(d / d \varepsilon)\right|_{\varepsilon=0}$ annihilates $a_{m}(A[\varepsilon \omega], B[0])$. The estimates in [GS] justify the following formal computation:

$$
\begin{aligned}
&\left.\sum_{n=0}^{\infty} \frac{d}{d \varepsilon}\right|_{\varepsilon=0} a_{n}(A[\varepsilon \omega], B[\varepsilon \omega]) t^{(n-m) / 2 l} \\
& \quad=\left.\sum_{n=0}^{\infty} \frac{d}{d \varepsilon}\right|_{\varepsilon=0} a_{n}(\underline{A[\varepsilon \omega]}, B[0]) t^{(n-m) / 2 l} \\
&\left.\sim \frac{d}{d \varepsilon}\right|_{\varepsilon=0} \operatorname{Tr} \exp \left(-t(\underline{A[\varepsilon \omega]})_{B}[0]\right) \\
&=-t \operatorname{Tr}\left\{\left(\left.\frac{d}{d \varepsilon}\right|_{\varepsilon=0}(\underline{A[\varepsilon \omega]})_{B}[0]\right) \exp \left(-t\left(A_{B}\right)[0]\right)\right\} \\
&=-t \operatorname{Tr}\left\{\left(\left.\frac{d}{d \varepsilon}\right|_{\varepsilon=0}\left(\mu\left(e^{-2 l \varepsilon \omega / h}\right) D[0]\right)^{h}\right)_{B[0]} \exp \left(-t\left(A_{B}\right)[0]\right)\right\} \\
&=2 l t \operatorname{Tr}\left\{\omega\left(A_{B} \exp \left(-t A_{B}\right)\right)[0]\right\} \\
&=-2 l t\left(\frac{d}{d t}\right) \operatorname{Tr}\left\{\omega \exp \left(-t\left(A_{B}\right)[0]\right)\right\} \\
& \sim \sum_{n=0}^{\infty}(m-n) a_{n}(\omega, A[0], B[0]) t^{(n-m) / 2 l},
\end{aligned}
$$

where the asymptotics are for $t \downarrow 0$. Here we have used the fact that $\exp \left(-t\left(A_{B}\right)[0]\right)$ is a smoothing operator for $t>0$, with the consequence that

$$
\operatorname{Tr}\left(U V e^{-t A_{B}}\right)=\operatorname{Tr}\left(V U e^{-t A_{B}}\right)
$$

as long as $U$ and $V$ are finite-order pseudodifferential operators, and $V$ commutes with $A_{B}$. Comparing coefficients for $n=m$, we get the result.

In the course of the proof, we have actually computed the conformal variation of $a_{n}(A, B)$ for every $n$.

2.11. Corollary. Under the assumptions of Theorem 2.10,

$$
\left.(d / d \varepsilon)\right|_{\varepsilon=0} a_{n}(A[\varepsilon \omega], B[\varepsilon \omega])=(m-n) a_{n}(\omega, A[0], B[0]) .
$$

The corollary shows that $a_{n}(A, B)$ is a conformal primitive, or integral, for $a_{n}(\omega, A, B)$ provided $n \neq m$. The following variational formula, which will be fundamental to our computations, shows that the functional determinant supplies the "missing" primitive for $a_{m}(\omega, A, B)$, at least when the conformal invariant $q(A, B)$ vanishes. 
2.12. Theorem. Suppose $(A, B)$ satisfies $1.2,1.6$, and 2.4. Let $(M, g[0])$ be a particular manifold with boundary together with a conformal class on which $\mathscr{N}\left(A_{B}\right)=0$, and let $\omega \in C^{\infty}(M)$. Then

$$
\left.(d / d \varepsilon)\right|_{\varepsilon=0} \zeta_{A[\varepsilon \omega], B[\varepsilon \omega]}^{\prime}(0)=2 l a_{m}(\omega, A[0], B[0]) .
$$

Proof. First assume that $\left(A_{B}\right)[0]$ is positive. By the conformal invariance of $q(A, B)$ and of $\#\left\{\lambda_{j}<0\right\}$ (Theorem 2.10), $\left(A_{B}\right)[\varepsilon \omega]$ is positive for all $\varepsilon \in \mathbb{R}$, so that the Mellin transform relates the zeta function to $\operatorname{Tr} \exp \left(-t A_{B}\right)$, without the modifications of (2.2). The estimates in [GS] allow us to conclude that $\left.(d / d \varepsilon)\right|_{\varepsilon=0} \zeta_{A, B}(s)$ is meromorphic, and that we can interchange the order of conformal variation and analytic continuation. For $\operatorname{Re} s$ large,

$$
\begin{aligned}
\left.\frac{d}{d \varepsilon}\right|_{\varepsilon=0} & \zeta_{A[\varepsilon \omega], B[\varepsilon \omega]}^{\prime}(s)=\left.\frac{d}{d s}\left(\frac{d}{d \varepsilon}\right)\right|_{\varepsilon=0} \zeta_{A[\varepsilon \omega], B[\varepsilon \omega]}(s) \\
= & \frac{d}{d s}\left\{\frac{1}{\Gamma(s)} \int_{0}^{\infty} t^{s-1}\left\{\left.\frac{d}{d \varepsilon}\right|_{\varepsilon=0} \operatorname{Tr} \exp \left(-t\left(A_{B}\right)[\varepsilon \omega]\right)\right\} d t\right\} \\
& =-\frac{d}{d s}\left\{\frac{2 l}{\Gamma(s)} \int_{0}^{\infty} t^{s}\left(\frac{d}{d t}\right) \operatorname{Tr}\left\{\omega \exp \left(-t\left(A_{B}\right)[0]\right)\right\} d t\right\} \\
& =\frac{d}{d s}\left\{\frac{2 l s}{\Gamma(s)} \int_{0}^{\infty} t^{s-1} \operatorname{Tr}\left\{\omega \exp \left(-t\left(A_{B}\right)[0]\right)\right\} d t\right\} .
\end{aligned}
$$

Here we have integrated by parts in $t$, and used the computations in (2.9). Analytically continuing this formula, the value at $s=0$ is the same as that of

$$
\frac{2 l}{\Gamma(s)} \int_{0}^{\infty} t^{s-1} \operatorname{Tr}\left\{\omega \exp \left(-t\left(A_{B}\right)[0]\right)\right\} d t
$$

that being $2 l a_{m}(\omega, A[0], B[0])$.

To dispense with the positivity assumption on $A_{B}$, note that we have proved the result for the positive operator $\left(A_{B}\right)^{2}$. (Recall Remark 2.9.) But $\zeta_{A^{2}, B^{(2)}}(s)=$ $\zeta_{A, B}(2 s)$, so $\zeta_{A^{2}, B^{(2)}}^{\prime}(0)=2 \zeta_{A, B}^{\prime}(0)$. But by a straightforward extension of [FG, Theorem 2.4] to boundary value problems, $a_{m}\left(\omega, A^{2}, B^{(2)}\right)=a_{m}(\omega, A, B)$.

2.13. Remark. The effect of zero spectrum on this argument is as follows. Since $q(A, B)$ is conformally invariant, nothing in (2.10) changes until we apply the $\varepsilon$-derivative; the trace on the third line becomes

$$
\operatorname{Tr}\left(\omega \exp \left(-t\left(A_{B}\right)[0]\right)-\mathscr{P}\right), \quad \mathscr{P}=\operatorname{Proj}_{\mathscr{N}(A[0]) \cap \mathscr{N}\left(\left(B \circ \mathrm{CD}_{2 l}\right)[0]\right)} \cdot
$$

The kernel function of $\omega\left\{\exp \left(-t\left(A_{B}\right)[0]\right)-\mathscr{P}\right\}$ is

$$
\omega(x)\left\{H(t, x, y)-\sum_{\lambda_{j}=0} \varphi_{j}(x) \otimes \varphi_{j}^{*}(y)\right\},
$$

where $H(t, x, y)$ is the kernel function of $\exp \left(-t\left(A_{B}\right)[0]\right)$, and $\left\{\varphi_{j}\right\}$ is an orthonormal basis of eigensections, $A[0] \varphi_{j}=\lambda_{j} \varphi_{j},\left(B \circ \mathrm{CD}_{2 l}\right)[0] \varphi_{j}=0$. The conclusion is that

$$
\left.\frac{d}{d \varepsilon}\right|_{\varepsilon=0} \zeta_{A[\varepsilon \omega], B[\varepsilon \omega]}^{\prime}(0)=2 l\left(a_{m}(\omega, A[0], B[0])-\int_{m} \omega(x) \sum_{\lambda_{j}=0}\left|\varphi_{j}(x)\right|^{2} d x\right) .
$$


Thus an explicit formula for the local heat invariant $a_{m}(\omega, A, B)$, or such a formula together with an explicit knowledge of the null space $\mathscr{N}\left(A_{B}\right)$ when this null space is nonzero, is sufficient for an understanding of the conformal behavior of the functional determinant. Note that an explicit knowledge of $\mathscr{N}\left(A_{B}\right)$ is not an unreasonable expectation: if the background metric has positive scalar curvature, there can be no null space for an elliptic boundary problem $Y_{B}$ based on the conformal Laplacian $Y$. For more general $(A, B)$ satisfying 2.4 , if $M$ and $\partial M$ are locally flat (for example, if $M$ is a standard flat halftorus), $\mathscr{N}\left(A_{B}\right)$ can be given explicitly in the background metric, and thus in conformal metrics by the conformal covariance law.

The strategy for computing the functional determinant within a conformal class will be to integrate the variational formula along a one-parameter family $g[\varepsilon \omega]=e^{2 \varepsilon \omega} g[0]$. The result will be a formula for the difference $\zeta_{A[\omega], B[\omega]}^{\prime}(0)-$ $\zeta_{A[0], B[0]}^{\prime}(0)$, that is, for the quotient of determinants $\left(\operatorname{det}\left(A_{B}\right)[\omega]\right) /\left(\operatorname{det}\left(A_{B}\right)[0]\right)$. The formulas involve integrals of differential polynomials in $\omega$, but such quantities cannot necessarily be reexpressed as integrals of scalar local invariants in the sense of Remark 1.9. For example, the quantity $\int \omega P \omega$, where $P$ is the Paneitz operator, appears in our formulas; it cannot, in general, be expressed as the integral of a local scalar invariant of $g[\omega]$. This phenomenon is one conformal manifestation of the nonlocal nature of the functional determinant. To express everything in terms of differential polynomials, at least via the current methods, it is very important that we stay within a conformal class.

The problem of computing $\operatorname{det}\left(A_{B}\right)[0]$, so that we have formulas for functional determinants instead of just quotients of such, may be approached separately; see $\S 7$.

\section{VARIATIONAL FORMULAS AND CONSEQUENCES OF THE CONFORMAL INDEX PROPERTY}

In this section, fix $\omega \in C^{\infty}(M)$, and again consider the variation $\left.(d / d \varepsilon)\right|_{\varepsilon=0}$ as the metric $g$ runs through the conformal curve $g[\varepsilon \omega]=e^{2 \varepsilon \omega} g[0]$ for a fixed (but arbitrary) $\omega \in C^{\infty}(M)$. We extend the definition of local scalar invariant to $f$-augmented local scalar invariants, $f \in C^{\infty}(M)$, by adding $d f$ (or $\tilde{d} f$ and $N f$ for boundary invariants) to the list of ingredients in Remark 1.9. (Note that suitable derivatives are also ingredients, so it is only the 0th derivative of $f$ that does not come into play.) When there is no chance of confusion as to the choice of manifold or measure, or when these choices are arbitrary, we shall sometimes abbreviate $\int_{M} \cdot d x$ by $\int \cdot$, and $\int_{\partial M} \cdot d y$ by $\oint$.

We begin by choosing a nonstandard basis of the interior invariants.

3.1. Lemma. With notation as in $\S 1$, the four quantities $|C|^{2}, Q, J^{2}, \Delta J$ span the space of level 4 local scalar $\mathrm{O}(m)$ invariants on $M$ for $m \geq 3$; for $m \geq 4$ they are a basis. If $m \geq 5$, these four quantities are also a basis of the level 4 local scalar $\mathrm{SO}(m)$-invariants. If $m=4$ and $C_{ \pm}$are the self-and anti-self-dual parts of $C$, the 5 quantities $\left|C_{+}\right|^{2},\left|C_{-}\right|^{2}, Q, J^{2}, \Delta J$ are a basis of the level 4 local scalar $\mathrm{SO}(4)$ invariants on $M$.

Proof. Let $m \geq 3$. By (1.1), $\Delta \tau$ is a scalar multiple of $\Delta J,|\rho|^{2}$ is a linear combination of $J^{2}$ and $|V|^{2}$, and $|R|^{2}$ is a linear combination of $J^{2},|V|^{2}$, and $|C|^{2} .|V|^{2}$ is a linear combination of $Q, J^{2}$, and $\Delta J$. By, e.g., [G1], the four quantities $|R|^{2},|\rho|^{2}, \tau^{2}, \Delta \tau$ span the $\mathrm{O}(m)$ invariants, and are a basis for 
$m \geq 4$. For $m \geq 5$, all $\mathrm{O}(m)$-irreducible summands of the vector bundle of which $R$ is a section are also $\mathrm{SO}(m)$-irreducible. For $m=4$, this is true except for the $\mathrm{O}(4)$-bundle of which $C$ is a section; this splits into two irreducible summands under $\mathrm{SO}(4)[\mathrm{S}]$, and this induces the splitting $C=C_{+}+C_{-}$.

Using the invariant theory of [BG], we can write down all the invariants that can appear in $a_{4}(f, A, B)$. In Table 3.1, we introduce abbreviations for some level 3 local scalar invariants on $\partial M . \operatorname{tr} L^{3}$ is an abbreviation for the local scalar invariant $L^{a}{ }_{b} L^{b}{ }_{c} L^{c}{ }_{a}$. For convenience, all indices are written as subscripts, the convention being that one index in each pair is raised before summing.

Let $f \in C^{\infty}(M)$ be an auxiliary function. In Table 3.2, we introduce abbreviations for some $f$-augmented level 3 local scalar invariants. Note that because the inward unit normal is extended to a collar as the tangent to a unit speed geodesic, the iterated partial derivatives $N \cdots N f$ agree with the iterated covariant derivatives $f_{\mid N \cdots N}=(\nabla \cdots \nabla f)_{N \cdots N}$.

From [BG, Lemma 2.3] and the above, we get

3.2. Lemma. Suppose that either $(A, B)$ is not orientation-sensitive or $m>4$. Under the analytic and naturality assumptions $1.2,1.6, a_{4}(f, A, B)$ has the

TABLE 3.1

\begin{tabular}{|c|c|c|}
\hline Abbreviation & Invariant & Index expression \\
\hline$X_{1}$ & $N \tau$ & $R_{i j i j \mid N}$ \\
\hline$X_{2}$ & $\tau H$ & $R_{i j i j} L_{a a}$ \\
\hline$X_{3}$ & $F H$ & $R_{a N a N} L_{b b}$ \\
\hline$X_{4}$ & $\langle G, L\rangle$ & $R_{a N b N} L_{a b}$ \\
\hline$X_{5}$ & $\langle T, L\rangle$ & $R_{c a c b} L_{a b}$ \\
\hline$X_{6}$ & $H^{3}$ & $L_{a a} L_{b b} L_{c c}$ \\
\hline$X_{7}$ & $H|L|^{2}$ & $L_{a a} L_{b c} L_{b c}$ \\
\hline$X_{8}$ & $\operatorname{tr} L^{3}$ & $L_{a b} L_{b c} L_{c a}$ \\
\hline
\end{tabular}

TABLE 3.2

\begin{tabular}{|c|c|c|}
\hline Abbreviation & Invariant & Index expression \\
\hline$Y_{1}(f)$ & $(N f) \tau$ & $f_{\mid N} R_{i j i j}$ \\
\hline$Y_{2}(f)$ & $\left(N^{2} f\right) H$ & $f_{\mid N N} L_{a a}$ \\
\hline$Y_{3}(f)$ & $(-\tilde{\Delta} f) H$ & $f_{: a a} L_{b b}$ \\
\hline$Y_{4}(f)$ & $(N f) H^{2}$ & $f_{\mid N} L_{a a} L_{b b}$ \\
\hline$Y_{5}(f)$ & $(N f) F$ & $f_{\mid N} R_{a N a N}$ \\
\hline$Y_{6}(f)$ & $\langle\tilde{\nabla} \tilde{\nabla} f, L\rangle$ & $f_{: a b} L_{a b}$ \\
\hline$Y_{7}(f)$ & $(N f)|L|^{2}$ & $f_{\mid N} L_{a b} L_{a b}$ \\
\hline$Y_{8}(f)$ & $N(-\Delta) f$ & $f_{\mid i i N}$ \\
\hline
\end{tabular}


form

$$
\begin{aligned}
a_{4}(f, A, B)= & \int f\left\{\alpha_{1,1}|C|^{2}+\alpha_{1,2} Q+\alpha_{1,3} J^{2}+\alpha_{1,4} \Delta J\right\} \\
& +\oint\left(f \sum_{\mu=1}^{8} \alpha_{2, \mu} X_{\mu}+\sum_{\nu=1}^{8} \alpha_{3, \nu} Y_{\nu}(f)\right)
\end{aligned}
$$

for some constants $\alpha_{\sigma, \mu}$ which depend only on the formal functorial expression for $(A, B)$, and on $m$. (In particular, they do not depend on the particular manifold or metric.) If $m=4$ and $(A, B)$ is orientation-sensitive, the same is true with $\alpha_{1,1}^{+}\left|C_{+}\right|^{2}+\alpha_{1,1}^{-}\left|C_{-}\right|^{2}$ in place of $\alpha_{1,1}|C|^{2}$ for constants $\alpha_{1,1}^{ \pm}$.

When $f=1$, the invariants $Y_{\nu}(f)$ vanish, and integration by parts gives

$$
\int \Delta J=\left(\oint X_{1}\right) / 2(m-1)
$$

Thus $a_{4}(A, B)$ has the form

$$
a_{4}(A, B)=\int\left\{\alpha_{1,1}|C|^{2}+\alpha_{1,2} Q+\alpha_{1,3} J^{2}\right\}+\oint \sum_{\mu=1}^{8} \tilde{\alpha}_{2, \mu} X_{\mu},
$$

where the $\tilde{\alpha}_{2,1}$ are constants with the same dependence as above. (We make the obvious adjustment if $m=4$ and $(A, B)$ is orientation-sensitive.) Under the conformal assumptions 2.4 , the number of undetermined coefficients in Lemma 3.2 and (3.2) is cut down considerably; the "axe" is the Conformal Index Theorem 2.10. To apply this, we need to know the conformal variations of the quantities involved.

3.3. Theorem. Let $m=4$. For the conformal variation above,

(a) $\left.(d / d \varepsilon)\right|_{\varepsilon=0}\left(|C|^{2} d x\right)[\varepsilon \omega]=0$. If $M$ is oriented,

$$
\left.(d / d \varepsilon)\right|_{\varepsilon=0}\left(\left|C_{ \pm}\right|^{2} d x\right)[\varepsilon \omega]=0 .
$$

(b)

$$
\left.\frac{d}{d \varepsilon}\right|_{\varepsilon=0} \int\left(J^{2} d x\right)[\varepsilon \omega]=2 \int \omega((\Delta J) d x)[0]+\frac{1}{3} \oint\left(\left\{-\omega X_{1}+Y_{1}(\omega)\right\} d y\right)[0] .
$$

(c)

$$
\begin{aligned}
& \left.\frac{d}{d \varepsilon}\right|_{\varepsilon=0} \int\left(|V|^{2} d x\right)[\varepsilon \omega]=2 \int \omega((\Delta J) d x)[0] \\
& \quad+\oint\left(\left\{-\frac{1}{3} \omega X_{1}-\frac{1}{6} Y_{1}(\omega)-Y_{3}(\omega)+Y_{5}(\omega)+Y_{6}(\omega)\right\} d y\right)[0]
\end{aligned}
$$

(d)

$$
\begin{aligned}
\left.\frac{d}{d \varepsilon}\right|_{\varepsilon=0} \int(\Delta J d x)[\varepsilon \omega] & =\left.\frac{d}{d \varepsilon}\right|_{\varepsilon=0} \oint\left(J_{\mid N} d y\right)[\varepsilon \omega] \\
& =\oint\left(\left\{-\frac{1}{3} Y_{1}(\omega)-Y_{8}(\omega)\right\} d y\right)[0]
\end{aligned}
$$


(e)

$$
\begin{gathered}
\left.\frac{1}{2}\left(\frac{d}{d \varepsilon}\right)\right|_{\varepsilon=0} \int(Q d x)[\varepsilon \omega]=\oint(\mathscr{S}(\omega) d y)[0] \\
\text { where } \mathscr{S}(\omega)=\frac{1}{3} Y_{1}(\omega)+Y_{3}(\omega)-Y_{5}(\omega)-Y_{6}(\omega)-\frac{1}{2} Y_{8}(\omega)
\end{gathered}
$$

Proof. (a) was already remarked as (1.4). (The statements about $C_{ \pm}$follow from the fact that the splitting into $C_{+}$and $C_{-}$is conformally invariant.) For (b), we use (1.2) and integrate by parts to get

$$
\begin{aligned}
\left.\frac{d}{d \varepsilon}\right|_{\varepsilon=0} \int\left(J^{2} d x\right)[\varepsilon \omega] & =2 \int J(\Delta \omega) d x=2 \int\langle d J, d \omega\rangle d x+2 \oint J \omega_{\mid N} d y \\
& =2 \int \omega(\Delta J) d x-2 \oint J_{\mid N} \omega d y+2 \oint J \omega_{\mid N} d y,
\end{aligned}
$$

where everything after the first "=" sign has an implicit [0] (is evaluated in $g[0])$.

For convenience in the rest of the proof, we write all indices as subscripts; one copy of each repeated index should be raised before summing. For (c), we use (1.3) and the Bianchi identity $V_{i j \mid i}=J_{\mid j}$, and integrate by parts:

$$
\begin{aligned}
\left.\frac{d}{d \varepsilon}\right|_{\varepsilon=0} \int\left(|V|^{2} d x\right)[\varepsilon \omega] & =-2 \int\langle V, \nabla \nabla \omega\rangle d x \\
& =2 \int\langle d J, d \omega\rangle d x+2 \oint V_{i N} \omega_{\mid i} d y \\
& =2 \int \omega \Delta J d x-2 \oint J_{\mid N} \omega d y+2 \oint V_{i N} \omega_{\mid i} d y .
\end{aligned}
$$

Again, everything after the first "=" sign is evaluated in $g[0]$. But

$$
V_{N i} \omega_{\mid i}=V_{N N} \omega_{\mid N}+V_{N a} \omega_{: a},
$$

and

$$
\oint V_{N a} \omega: a=-\oint V_{a N: a} \omega=-\frac{1}{2} \oint \rho_{a N: a} \omega .
$$

By [BG, Lemma A.1(b)],

$$
\rho_{a N: a}=H_{: a a}-L_{a b: a b} .
$$

Since $V_{N N}=\frac{1}{2} F-\frac{1}{12} \tau$, integration by parts over $\partial M$ (which has no boundary) gives

$$
\oint V_{N i} \omega_{\mid i}=\oint\left(\frac{1}{2} Y_{5}(\omega)-\frac{1}{12} Y_{1}(\omega)-\frac{1}{2} Y_{3}(\omega)+\frac{1}{2} Y_{6}(\omega)\right) .
$$

This and $J=\tau / 6$ give (c).

The first equality in (d) is obtained by integrating by parts; the second is Lemma 3.4(a). For the proof of (e), we use (1.7):

$$
\begin{aligned}
\left.\frac{1}{2}\left(\frac{d}{d \varepsilon}\right)\right|_{\varepsilon=0} \int(Q d x)[\varepsilon \omega]=\frac{1}{2} \int(P \omega) d x \\
=\frac{1}{2} \int \omega_{\mid i i j j} d x-\int[\{J-2 V \cdot\} d \omega]_{j \mid j} d x \\
=-\frac{1}{2} \oint \omega_{\mid i i N} d y+\oint[\{J-2 V \cdot\} d \omega]_{N} d y \\
=-\frac{1}{2} \oint \omega_{\mid i i N} d y+\oint\left(J \omega_{\mid N}-2 V_{N i} \omega_{\mid i}\right) d y
\end{aligned}
$$


(3.3) now finishes the proof. Alternatively, we could use the definition (1.5) of $Q$ together with parts (b)-(d) to derive (e).

We shall also need the conformal variations of the boundary invariants that do not automatically vanish for $\omega=1$. The following can be read off from the variational formulas in the appendix to $[\mathrm{BG}]$. (Note the differences in sign conventions.)

\subsection{Lemma. Let}

$$
X_{i}^{\prime}(\omega)=\left.\frac{d}{d \varepsilon}\right|_{\varepsilon=0}\left(e^{3 \varepsilon \omega} X_{i}[\varepsilon \omega]\right)=\left.\frac{d}{d \varepsilon}\right|_{\varepsilon=0} X_{i}[\varepsilon \omega]+3 \omega X_{i}[0] .
$$

Then:

(a) $X_{1}^{\prime}(\omega)=-2 Y_{1}(\omega)-2(m-1) Y_{8}(\omega)$.

(b) $X_{2}^{\prime}(\omega)=-(m-1) Y_{1}(\omega)-2(m-1) Y_{2}(\omega)-2(m-1) Y_{3}(\omega)+$ $2(m-1) Y_{4}(\omega)$.

(c) $X_{3}^{\prime}(\omega)=-(m-1) Y_{2}(\omega)-Y_{3}(\omega)+Y_{4}(\omega)-(m-1) Y_{5}(\omega)$.

(d) $X_{4}^{\prime}(\omega)=-Y_{2}(\omega)-Y_{5}(\omega)-Y_{6}(\omega)+Y_{7}(\omega)$.

(e) $X_{5}^{\prime}(\omega)=-Y_{1}(\omega)-Y_{3}(\omega)+Y_{4}(\omega)+2 Y_{5}(\omega)-(m-3) Y_{6}(\omega)+$ $(m-3) Y_{7}(\omega)$.

(f) $X_{6}^{\prime}(\omega)=-3(m-1) Y_{4}(\omega)$.

(g) $X_{7}^{\prime}(\omega)=-2 Y_{4}(\omega)-(m-1) Y_{7}(\omega)$.

(h) $X_{8}^{\prime}(\omega)=-3 Y_{7}(\omega)$.

We retain the "prime" notation of Lemma 3.4 to derive some straightforward consequences in the next two lemmas.

\subsection{Lemma. Let}

$$
\begin{aligned}
& \mathscr{L}_{4}=-\frac{1}{m-1} X_{2}+X_{3}-(m-3) X_{4}+X_{5}, \\
& \mathscr{L}_{5}=-\frac{2}{3(m-1)} X_{6}+X_{7}-\frac{m-1}{3} X_{8} .
\end{aligned}
$$

Then $\mathscr{L}_{s}^{\prime}(\omega)=0, s=4,5$.

3.6. Lemma. Let $m=4$, and let

$$
S=-\frac{1}{12} X_{1}+\frac{1}{6} X_{2}-X_{4}+\frac{1}{9} X_{6}-\frac{1}{3} X_{8}
$$

Then $S^{\prime}(\omega)=-\mathscr{S}(\omega)$, where $\mathscr{S}(\omega)$ is as in Lemma 3.3(e).

We can now harvest the consequences of the conformal index property, reducing the number of undetermined coefficients from the 20 in Lemma 3.2 to just 13 under the conformal assumptions.

3.7. Theorem. Let $m=4$, and suppose $(A, B)$ satisfies $1.2,1.6$, and 2.4. Suppose that $(A, B)$ is not orientation-sensitive. Then $a_{4}(f, A, B)$ has the 
form

$$
\begin{aligned}
a_{4}(f, A, B)= & \beta_{1} \int f|C|^{2}+\beta_{2}\left\{\frac{1}{2} \int f Q+\oint f S\right\} \\
& +\beta_{3}\left\{\int f \Delta J-\frac{1}{6} \oint f X_{1}\right\}+\beta_{4} \oint f \mathscr{L}_{4}+\beta_{5} \oint f \mathscr{L}_{5} \\
& +\oint \sum_{\nu=1}^{8} \kappa_{\nu} Y_{\nu}(f),
\end{aligned}
$$

where the constants $\beta_{\mu}, \mu=1, \ldots, 5$, and $\kappa_{\nu}, \nu=1, \ldots, 8$, depend only on the formal functorial expression for $(A, B)$. In particular,

$$
a_{4}(A, B)=\beta_{1} \int|C|^{2}+\beta_{2}\left\{\frac{1}{2} \int Q+\oint S\right\}+\beta_{4} \oint \mathscr{L}_{4}+\beta_{5} \oint \mathscr{L}_{5} .
$$

If $(A, B)$ is orientation-sensitive, the same is true with $\beta_{1,+}\left|C_{+}\right|^{2}+\beta_{1,-}\left|C_{-}\right|^{2}$ in place of $\beta_{1}|C|^{2}$ in each formula.

Proof. Changing basis in the formula of Lemma 3.2, we may write $a_{4}(f, A, B)$ in the form

$$
\begin{aligned}
& \beta_{1} \int f|C|^{2}+\beta_{2}\left\{\frac{1}{2} \int f Q+\oint f S\right\}+\beta_{3}\left\{\int f \Delta J-\frac{1}{6} \oint f X_{1}\right\}+c \int f J^{2} \\
& +\oint f\left\{\beta_{4} \mathscr{L}_{4}+\beta_{5} \mathscr{L}_{5}+\gamma_{1} X_{1}+\gamma_{2} X_{2}+\gamma_{3} X_{3}+\gamma_{4} X_{4}+\gamma_{6} X_{6}+\gamma_{8} X_{8}\right\} \\
& +\oint \sum_{\nu=1}^{8} \kappa_{\nu} Y_{\nu}(f)
\end{aligned}
$$

for some universal constants $\beta_{i}, c, \gamma_{j}$. In particular, by (3.1),

$$
\begin{aligned}
a_{4}(A, B)= & \beta_{1} \int|C|^{2}+\beta_{2}\left(\frac{1}{2} \int Q+\oint S\right)+c \int J^{2}+\beta_{4} \oint \mathscr{L}_{4} \\
& +\beta_{5} \oint \mathscr{L}_{5}+\sum_{j=1,2,3,4,6,8} \oint \gamma_{j} X_{j} .
\end{aligned}
$$

By Lemmas 3.3-3.6,

$$
\begin{aligned}
\left.\frac{d}{d \varepsilon}\right|_{\varepsilon=0} & a_{4}(A[\varepsilon \omega], B[\varepsilon \omega]) \\
= & 2 c \int \omega(\Delta J) d x-\frac{1}{3} c \oint \omega X_{1}+\left(\frac{1}{3} c-2 \gamma_{1}-3 \gamma_{2}\right) \oint Y_{1}(\omega) \\
& +\left(-6 \gamma_{2}-3 \gamma_{3}-\gamma_{4}\right) \oint Y_{2}(\omega)+\left(-6 \gamma_{2}-\gamma_{3}\right) \oint Y_{3}(\omega) \\
& +\left(6 \gamma_{2}+\gamma_{3}-9 \gamma_{6}\right) \oint Y_{4}(\omega)+\left(-3 \gamma_{3}-\gamma_{4}\right) \oint Y_{5}(\omega)-\gamma_{4} \oint Y_{6}(\omega) \\
& +\left(\gamma_{4}-3 \gamma_{8}\right) \oint Y_{7}(\omega)-6 \gamma_{1} \oint Y_{8}(\omega) .
\end{aligned}
$$

By the linear independence of the invariants $\oint Y_{j}(\omega)$, we conclude that $c=$ $\gamma_{1}=\gamma_{2}=\gamma_{3}=\gamma_{4}=\gamma_{6}=\gamma_{8}=0$.

It is now time to clarify the notion of conformal primitive. 
3.8. Definition. A real functional $\mathscr{P}$ on a conformal class $\langle g[0]\rangle$ on $M$ is smooth if the functions

$$
\mathbb{R}^{N} \rightarrow \mathbb{R}, \quad \varepsilon=\left(\varepsilon_{1}, \ldots, \varepsilon_{N}\right) \mapsto \mathscr{P}\left(g\left[\sum_{i=1}^{N} \varepsilon_{i} \omega_{i}\right]\right)
$$

are $C^{\infty}$ for all $N \in \mathbb{Z}^{+}$and functions $\omega_{i} \in C^{\infty}(M) . \mathscr{P}$ has degree $n \in \mathbb{Z}^{+}$if the maps (3.5) are polynomial in $\varepsilon$ of degree $n$, and we use the terminology linear, quadratic, etc. for degree $1,2, \ldots$ A map $\mathscr{R}$ from $\langle g[0]\rangle$ to $C^{\infty}(M)$ is smooth (resp. has degree $n$ ) if $g \mapsto \mathscr{R}(g[\omega])(x)$ is smooth (resp. is polynomial of degree $n$ in $\varepsilon$ ) for each $x \in M$, and similarly for maps to $C^{\infty}(\partial M)$.

3.9. Definition. Let $\mathscr{P}$ and $\mathscr{T}$ be smooth functionals on a conformal class $\langle g[0]\rangle$ on $M . \mathscr{P}$ is a conformal primitive for $\mathscr{T}$ if

$$
\left.(d / d \varepsilon)\right|_{\varepsilon=0} \mathscr{P}(g[\eta+\varepsilon \omega])=\mathscr{T}(g[\eta])
$$

for all $\eta, \omega \in C^{\infty}(M)$. If in addition, a base metric $g[0]$ is given and $\mathscr{P}(g[0])=0, \mathscr{P}$ is a base-pointed conformal primitive for $\mathscr{T}$.

3.10. Remark. A base-pointed conformal primitive $\mathscr{P}$ for $\mathscr{T}$, if it exists, is unique, since the curve $\alpha(\varepsilon)=\mathscr{P}(g[\varepsilon \omega])$ solves the initial value problem $(d / d \varepsilon) \alpha(\varepsilon)=\mathscr{T}(g[\varepsilon \omega]), \alpha(0)=0$. If a functional $\mathscr{T}$ and a prospective conformal primitive $\mathscr{P}$ are given by universal formulas, it is sufficient to prove (3.6) at $\eta=0$, since universality allows us to replace $g[0]$ by $g[\eta]$. If $\mathscr{T}(g[\omega])$ is a homogeneous polynomial functional of degree $n>0$, then $\mathscr{T}(g[\omega]) / n$ is a base-pointed conformal primitive for $\mathscr{T}(g[\omega])$. Thus a decomposition of a given functional into homogeneous polynomial functionals is sufficient information for the computation of a base-pointed conformal primitive. We shall sometimes use the abbreviation $\mathscr{T}[\omega]$ for $\mathscr{T}(g[\omega])$.

Motivated by Remark 3.10 , we go on to compute the higher conformal variations of the local invariants in Theorem 3.7. The formulas for $\nabla[\omega]$, $R[\omega], L[\omega], \tilde{\nabla}[\omega]$, and $N[\omega]$ show that each term in that expression for $a_{4}(\omega, A[\omega], B[\omega])$ is polynomial of degree $\leq 4$; this will also emerge from our calculations, so we omit the abstract proof. We first introduce abbreviations for some $f$-augmented local invariants which are quadratic and cubic in $f$.

TABLE 3.3

\begin{tabular}{|c|c|c|}
\hline Abbreviation & Invariant & Index expression \\
\hline$Z_{1}(f, f)$ & $(N f) N^{2} f$ & $f_{\mid N} f_{\mid N N}$ \\
\hline$Z_{2}(f, f)$ & $(N f)(-\tilde{\Delta}) f$ & $f_{\mid N} f_{: a a}$ \\
\hline$Z_{3}(f, f)$ & $(N f)^{2} H$ & $f_{\mid N} f_{\mid N} L_{a a}$ \\
\hline$Z_{4}(f, f)$ & $|\tilde{d} f|^{2} H$ & $f_{: a} f_{: a} L_{b b}$ \\
\hline$Z_{5}(f, f)$ & $\langle\tilde{d} f \otimes \tilde{d} f, L\rangle$ & $f_{: a} f_{: b} L_{a b}$ \\
\hline$Z_{6}(f, f)$ & $\langle\tilde{d} f, \tilde{d}(N f)\rangle$ & $f_{: a}\left(f_{\mid N}\right)_{: a}$ \\
\hline$E_{1}(f, f, f)$ & $(N f)|\tilde{d} f|^{2}$ & $f_{\mid N} f_{: a} f_{: a}$ \\
\hline$E_{2}(f, f, f)$ & $(N f)^{3}$ & $f_{\mid N} f_{\mid N} f_{\mid N}$ \\
\hline
\end{tabular}


The notation $Z_{i}(f, f)$ indicates that $Z_{i}$ can actually be thought of as a quadratic form $Z_{i}\left(f_{1}, f_{2}\right)$ after polarization; similarly for the cubic form determined by $E_{j}(f, f, f)$. Lemmas 3.11-3.18 immediately following are obtained by direct computation with the variational formulas and identities of [BG, Appendix], and integration by parts.

\subsection{Lemma. Let}

$$
Y_{i}^{\prime}(\omega, \omega)=\left.\frac{d}{d \varepsilon}\right|_{\varepsilon=0}\left(e^{3 \varepsilon \omega} Y_{i}(\omega)[\varepsilon \omega]\right)=\left.\frac{d}{d \varepsilon}\right|_{\varepsilon=0} Y_{i}(\omega)[\varepsilon \omega]+3 \omega Y_{i}(\omega)[0] .
$$

Then:
(a) $\quad Y_{1}^{\prime}(\omega, \omega)=-2(m-1) Z_{1}(\omega, \omega)-2(m-1) Z_{2}(\omega, \omega)$ $+2(m-1) Z_{3}(\omega, \omega)$.
(b) $Y_{2}^{\prime}(\omega, \omega)=-(m-1) Z_{1}(\omega, \omega)-Z_{3}(\omega, \omega)+Z_{4}(\omega, \omega)$.
(c) $Y_{3}^{\prime}(\omega, \omega)=-(m-1) Z_{2}(\omega, \omega)+(m-3) Z_{4}(\omega, \omega)$.
(d) $Y_{4}^{\prime}(\omega, \omega)=-2(m-1) Z_{3}(\omega, \omega)$.
(e) $Y_{5}^{\prime}(\omega, \omega)=-(m-1) Z_{1}(\omega, \omega)-Z_{2}(\omega, \omega)+Z_{3}(\omega, \omega)$.
(f) $Y_{6}^{\prime}(\omega, \omega)=-Z_{2}(\omega, \omega)+Z_{4}(\omega, \omega)-2 Z_{5}(\omega, \omega)$.
(g) $Y_{7}^{\prime}(\omega, \omega)=-2 Z_{3}(\omega, \omega)$.
(h) $Y_{8}^{\prime}(\omega, \omega)=2(m-3) Z_{1}(\omega, \omega)-2 Z_{2}(\omega, \omega)+2 Z_{3}(\omega, \omega)$ $+2(m-2) Z_{5}(\omega, \omega)+2(m-2) Z_{6}(\omega, \omega)$.

3.12. Lemma. For all $f, \omega \in C^{\infty}(M)$,

(a) $\oint Z_{6}(f, f)=-\oint Z_{2}(f, f)$.

(b) $\oint f Z_{6}(f, f)=-\oint f Z_{2}(f, f)-\oint E_{1}(f, f, f)$.

(c)

$$
\begin{aligned}
& \left.\frac{d}{d \varepsilon}\right|_{\varepsilon=0}\left(\oint\left(Y_{8}(\omega) d y\right)[\varepsilon \omega]\right)=(m-4) \oint \omega\left(Y_{8}(\omega) d y\right)[0] \\
& \quad+\oint\left\{2(m-3) Z_{1}(\omega, \omega)-2(m-1) Z_{2}(\omega, \omega)\right. \\
& \left.\quad+2 Z_{3}(\omega, \omega)+2(m-2) Z_{5}(\omega, \omega)\right\} .
\end{aligned}
$$

3.13. Lemma. Let

$$
\begin{aligned}
Z_{i}^{\prime}(\omega, \omega, \omega) & =\left.\frac{d}{d \varepsilon}\right|_{\varepsilon=0}\left(e^{3 \varepsilon \omega} Z_{i}(\omega, \omega)[\varepsilon \omega]\right) \\
& =\left.\frac{d}{d \varepsilon}\right|_{\varepsilon=0} Z_{i}(\omega, \omega)[\varepsilon \omega]+3 \omega Z_{i}(\omega, \omega)[0]
\end{aligned}
$$

Then:

(a) $Z_{1}^{\prime}(\omega, \omega, \omega)=E_{1}(\omega, \omega, \omega)-E_{2}(\omega, \omega, \omega)$.

(b) $Z_{2}^{\prime}(\omega, \omega, \omega)=(m-3) E_{1}(\omega, \omega, \omega)$.

(c) $Z_{3}^{\prime}(\omega, \omega, \omega)=-(m-1) E_{2}(\omega, \omega, \omega)$.

(d) $Z_{4}^{\prime}(\omega, \omega, \omega)=-(m-1) E_{1}(\omega, \omega, \omega)$.

(e) $Z_{5}^{\prime}(\omega, \omega, \omega)=-E_{1}(\omega, \omega, \omega)$.

(f) $Z_{6}^{\prime}(\omega, \omega, \omega)=-E_{1}(\omega, \omega, \omega)$. 
3.14. Lemma. The quantities

$$
\begin{aligned}
E_{i}^{\prime}(\omega, \omega, \omega, \omega) & =\left.\frac{d}{d \varepsilon}\right|_{\varepsilon=0}\left(e^{3 \varepsilon \omega} E_{i}(\omega, \omega, \omega)[\varepsilon \omega]\right) \\
& =\left.\frac{d}{d \varepsilon}\right|_{\varepsilon=0} Z_{i}(\omega, \omega, \omega)[\varepsilon \omega]+3 \omega Z_{i}(\omega, \omega, \omega)[0]
\end{aligned}
$$

vanish identically.

3.15. Lemma. For $m \geq 2$, let

$$
\begin{aligned}
l_{1}(\omega)= & -Y_{4}(\omega)+(m-1) Y_{7}(\omega) \\
l_{2}(\omega)= & -Y_{1}(\omega)-(m-3) Y_{2}(\omega)+Y_{3}(\omega)-\frac{1}{m-1} Y_{4}(\omega)+(m-1) Y_{5}(\omega), \\
l_{3}(\omega)= & \frac{m^{2}-3 m-2}{m-1} Y_{2}(\omega)-\frac{2 m}{m-1} Y_{3}(\omega)-\frac{m^{2}-5 m+2}{(m-1)^{2}} Y_{4}(\omega) \\
& -(m-4) Y_{5}(\omega)+(m-2) Y_{6}(\omega)+Y_{8}(\omega) .
\end{aligned}
$$

Then $l_{s}^{\prime}(\omega, \omega)=0$ for $s=1,2$, and

$$
\left.\frac{d}{d \varepsilon}\right|_{\varepsilon=0} \oint\left(l_{3}(\omega) d y\right)[\varepsilon \omega]=(m-4) \oint \omega\left(l_{3}(\omega) d y\right)[0]
$$

In particular, if $m=4$, then

$$
\left.\frac{d}{d \varepsilon}\right|_{\varepsilon=0} \oint\left(l_{s}(\omega) d y\right)[\varepsilon \omega]=0, \quad s=1,2,3 .
$$

\subsection{Lemma. Let}

$$
\mathscr{A}=X_{6}-(m-1)^{2} X_{8}
$$

Then $\mathscr{A}^{\prime \prime}(\omega, \omega)=0$.

3.17. Lemma. Let

$$
\begin{aligned}
& q_{1}(\omega)=Y_{3}(\omega)-(m-1) Y_{6}(\omega), \\
& q_{2}(\omega)=(m-3) Y_{1}(\omega)+(m-3)(m-2) Y_{2}(\omega)-2(m-2) Y_{3}(\omega), \\
& q_{3}(\omega)=(m-1) Y_{1}(\omega)-(m-1)(m-2) Y_{2}(\omega)+2(m-2) Y_{4}(\omega) .
\end{aligned}
$$

Then $q_{s}^{\prime \prime}(\omega, \omega, \omega)=0, s=1,2,3$, with the result that

$$
\left.\frac{d^{2}}{d \varepsilon^{2}}\right|_{\varepsilon=0}\left(\oint\left(q_{s} d y\right)[\varepsilon \omega]\right)=0, \quad m=4 .
$$

3.18. Lemma. If $f \in C^{\infty}(M)$, then on $\partial M$,

$$
\begin{aligned}
f_{\mid i}{ }^{i} N= & f_{\mid N N N}+\left(f_{\mid N}\right): a^{a}+2 \tilde{\nabla}^{a}\left(L_{a b} \tilde{\nabla}^{b} f\right) \\
& -H: a f:{ }^{a}-F f_{\mid N}-|L|^{2} f_{\mid N}-H f_{\mid N N} .
\end{aligned}
$$

The following lemma is a consequence of Lemmas 3.4, 3.11, 3.12, and 3.13.

3.19. Lemma. If $m=4$ and $S$ is as in Lemma 3.6, then

$$
\left.\frac{d^{2}}{d \varepsilon^{2}}\right|_{\varepsilon=0}\left(\oint \omega(S d y)[\varepsilon \omega]-\frac{1}{3} \oint Y_{3}(\omega)[\varepsilon \omega] d y[\varepsilon \omega]\right)=0
$$




\section{FORMULAS FOR THE QUOTIENT OF FUNCTIONAL DETERMINANTS}

Suppose we have a compact 4-manifold $M$ with boundary $\partial M$, and a basepointed conformal class $\langle g[0]\rangle$ is given. Suppose also that we have a boundary value problem $(A, B)$ satisfying the assumptions of Theorem 2.12. A change of basis in the formula of Theorem 3.7 allows us to write

$$
\begin{aligned}
a_{4}(\omega, & A, B)[\omega]=\beta_{1} \int \omega\left(|C|^{2} d x\right)[\omega] \\
+ & \beta_{2}\left\{\frac{1}{2} \int \omega(Q d x)[\omega]+\oint\left(\left(\omega S-\frac{1}{3} Y_{3}(\omega)\right) d y\right)[\omega]\right\} \\
+ & \beta_{3}\left\{\int \omega((\Delta J) d x)[\omega]+\frac{1}{6} \oint\left(\left(Y_{1}(\omega)-\omega X_{1}\right) d y\right)[\omega]\right\} \\
+ & \beta_{4} \oint \omega\left(\mathscr{L}_{4} d y\right)[\omega]+\beta_{5} \oint \omega\left(\mathscr{L}_{5} d y\right)[\omega] \\
+ & \sum_{i=1}^{3} \lambda_{i} \oint\left(l_{i}(\omega) d y\right)[\omega]+\sum_{j=1}^{3} \sigma_{j} \oint\left(q_{j}(\omega) d y\right)[\omega] \\
& +c_{3} \oint\left(Y_{3}(\omega) d y\right)[\omega]+c_{4} \oint\left(Y_{4}(\omega) d y\right)[\omega],
\end{aligned}
$$

where $\beta_{\nu}, \lambda_{i}, \sigma_{j}, c_{3}, c_{4}$ are universal constants depending on the universal polynomial expression for $(A, B)$.

By Theorem 2.12, we can find a formula for

$$
-\log \left|\operatorname{det}\left(A_{B}\right)\right|[\omega]+\log \left|\operatorname{det}\left(A_{B}\right)\right|[0]
$$

by finding a base-pointed conformal primitive for each term in (4.1). By Theorem 2.10, the sign $(-1)^{\#\left\{\lambda_{j}<0\right\}}$ of $\operatorname{det} A_{B}$ is conformally invariant, so this gives a formula for $\operatorname{det}\left(A_{B}\right)[\omega] / \operatorname{det}\left(A_{B}\right)[0]$.

4.1. Lemma. In (4.1), the $\beta_{\nu}$ terms for $\nu=1,4,5$ and the $\lambda_{i}$ terms for $i=1,2,3$ have base-pointed conformal primitives

$$
\begin{array}{cc}
\beta_{1} \int \omega\left(|C|^{2} d x\right)[0], & \beta_{4} \oint \omega\left(\mathscr{L}_{4} d y\right)[0], \\
\beta_{5} \oint \omega\left(\mathscr{L}_{5} d y\right)[0], & \lambda_{i} \oint\left(l_{i}(\omega) d y\right)[0]
\end{array}
$$

respectively.

Proof. By Lemmas 3.3(a) and 3.5, $|C|^{2} d x, \mathscr{L}_{4} d y$, and $\mathscr{L}_{5} d y$ are conformal invariants in dimension 4; thus the relevant $\beta_{\nu}$ terms in (4.1) are linear on the conformal class $\langle g[0]\rangle$. By Lemma 3.15, the $\lambda_{i}$ terms are also linear. We now apply Remark 3.10.

4.2. Lemma. A base-pointed conformal primitive for $\int \omega(Q d x)[\omega]$ is

$$
\mathscr{B}[\omega]:=\frac{1}{2} \int \omega(P[0] \omega) d x[0]+\int \omega(Q d x)[0] .
$$

Proof. If $\eta, \omega \in C^{\infty}(M)$,

$$
\left.\frac{d}{d \varepsilon}\right|_{\ell=0} \mathscr{B}[\eta+\varepsilon \omega]=\frac{1}{2} \int\{\eta(P[0] \omega)+\omega(P[0] \eta)\} d x[0]+\int \omega(Q d x)[0] .
$$


Evaluation at $\eta=\varepsilon_{0} \omega$ shows that the curve $\mathscr{B}[\varepsilon \omega]$ satisfies the initial value problem

$$
\left.\frac{d}{d \varepsilon}\right|_{\varepsilon=\varepsilon_{0}} \mathscr{B}[\varepsilon \omega]=\int\left\{\varepsilon_{0} \omega(P[0] \omega)+\omega Q[0]\right\} d x[0], \quad \mathscr{B}[0]=0 .
$$

But by (1.7), the right side of this ordinary differential equation is

$$
\int \omega(Q d x)\left[\varepsilon_{0} \omega\right]
$$

as desired.

4.3. Lemma. A base-pointed conformal primitive for

$$
\mathscr{C}[\omega]:=\oint\left(\left(\omega S-\frac{1}{3} Y_{3}(\omega)\right) d y\right)[\omega]
$$

is

$$
\begin{aligned}
\oint \omega(S d y)[0] & -\frac{1}{3} \oint\left(Y_{3}(\omega) d y\right)[0]-\frac{1}{2} \oint \omega(\mathscr{S}(\omega) d y)[0] \\
& +\frac{1}{2} \oint\left(Z_{2}(\omega, \omega) d y\right)[0]-\frac{1}{6} \oint\left(Z_{4}(\omega, \omega) d y\right)[0]
\end{aligned}
$$

Proof. By Lemma 3.19, $\mathscr{C}[\omega]$ is a quadratic functional. By Remark 3.10, its base-pointed conformal primitive is

$$
\oint \omega(S d y)[0]-\frac{1}{3} \oint\left(Y_{3}(\omega) d y\right)[0]+\frac{1}{2} \oint\left(\omega S^{\prime}(\omega)[0]-\frac{1}{3} Y_{3}^{\prime}(\omega, \omega)[0]\right) d y[0]
$$

The last line of this is computed using Lemmas 3.6 and 3.11.

4.4. Lemma. The $\beta_{3}$ term in (4.1) has base-pointed conformal primitive

$$
\frac{1}{2} \beta_{3} \int\left\{\left(J^{2} d x\right)[\omega]-\left(J^{2} d x\right)[0]\right\}
$$

Proof. This is a restatement of Lemma 3.3(b).

4.5. Lemma. The sum of the $\sigma_{j}, c_{3}$, and $c_{4}$ terms in (4.1) has base-pointed conformal primitive

$$
\begin{aligned}
& \sigma_{1}\left\{\oint\left(q_{1}(\omega) d y\right)[0]+\oint\left(\left(-Z_{4}+3 Z_{5}\right)(\omega, \omega) d y\right)[0]\right\} \\
& +\sigma_{2}\left\{\oint\left(q_{2}(\omega) d y\right)[0]+\oint\left(\left(-6 Z_{1}+3 Z_{2}+2 Z_{3}-Z_{4}\right)(\omega, \omega) d y\right)[0]\right\} \\
& +\sigma_{3}\left\{\oint\left(q_{3}(\omega) d y\right)[0]+\oint\left(\left(-9 Z_{2}-3 Z_{4}\right)(\omega, \omega) d y\right)[0]\right\} \\
& +c_{3}\left\{\oint\left(Y_{3}(\omega) d y\right)[0]+\oint\left(\left(-\frac{3}{2} Z_{2}+\frac{1}{2} Z_{4}\right)(\omega, \omega) d y\right)[0]\right. \\
& +c_{4}\left\{\oint\left(Y_{4}(\omega) d y\right)[0]-3 \oint\left(Z_{3}(\omega, \omega) d y\right)[0]+3 \oint\left(E_{2}(\omega, \omega, \omega) d y\right)[0]\right\}
\end{aligned}
$$


Proof. By Lemma 3.17, the $\sigma_{j}$ terms in (4.1) are quadratic on the conformal class $\langle g[0]\rangle$, and by Lemmas $3.11,3.13$, and 3.14, the $c_{4}$ and $c_{7}$ terms are cubic. We now apply Remark 3.10, using Lemmas 3.11 and 3.13 to compute expansions into homogeneous polynomial terms.

We collect all this information in the following.

4.6. Theorem. Under the assumptions of Theorem 2.12, if $(A, B)$ is not orientation-sensitive,

$$
\begin{aligned}
-(2 l)^{-1} \log \frac{\operatorname{det}\left(A_{B}\right)[\omega]}{\operatorname{det}\left(A_{B}\right)[0]}=\beta_{1} \int \omega\left(|C|^{2} d x\right)[0] \\
+\beta_{2}\left\{\frac{1}{4} \int \omega(P[0] \omega) d x[0]\right. \\
\quad+\frac{1}{2} \int \omega(Q d x)[0]+\oint \omega(S d y)[0]-\frac{1}{3} \oint\left(Y_{3}(\omega) d y\right)[0] \\
\left.\quad-\frac{1}{2} \oint \omega(\mathscr{S}(\omega) d y)[0]+\oint\left(\left(\frac{1}{2} Z_{2}-\frac{1}{6} Z_{4}\right)(\omega, \omega) d y\right)[0]\right\} \\
+\frac{1}{2} \beta_{3} \int\left\{\left(J^{2} d x\right)[\omega]-\left(J^{2} d x\right)[0]\right\}+\beta_{4} \oint \omega\left(\mathscr{L}_{4} d y\right)[0] \\
+\beta_{5} \oint \omega\left(\mathscr{L}_{5} d y\right)[0]+\sum_{i=1}^{3} \lambda_{i} \oint\left(l_{i}(\omega) d y\right)[0] \\
+\sigma_{1}\left\{\oint\left(q_{1}(\omega) d y\right)[0]+\oint\left(\left(-Z_{4}+3 Z_{5}\right)(\omega, \omega) d y\right)[0]\right\} \\
+\sigma_{2}\left\{\oint\left(q_{2}(\omega) d y\right)[0]+\oint\left(\left(-6 Z_{1}+3 Z_{2}+2 Z_{3}-Z_{4}\right)(\omega, \omega) d y\right)[0]\right\} \\
+\sigma_{3}\left\{\oint\left(q_{3}(\omega) d y\right)[0]+\oint\left(\left(-9 Z_{2}-3 Z_{4}\right)(\omega, \omega) d y\right)[0]\right\} \\
+c_{3}\left\{\oint\left(Y_{3}(\omega) d y\right)[0]+\oint\left(\left(-\frac{3}{2} Z_{2}+\frac{1}{2} Z_{4}\right)(\omega, \omega) d y\right)[0]\right. \\
+c_{4}\left\{\oint\left(Y_{4}(\omega) d y\right)[0]-3 \oint\left(Z_{3}(\omega, \omega) d y\right)[0]+3 \oint\left(E_{2}(\omega, \omega, \omega) d y\right)[0]\right\} .
\end{aligned}
$$

If $(A, B)$ is orientation-sensitive, $\beta_{1} \int \omega\left(|C|^{2} d x\right)[0]$ should be replaced by

$$
\beta_{1,+} \int \omega\left(\left|C_{+}\right|^{2} d x\right)[0]+\beta_{1,-} \int \omega\left(\left|C_{-}\right|^{2} d x\right)[0] .
$$

If $\mathscr{P}_{\lambda}$ is the functional (2.4), then

$$
\begin{aligned}
-(2 l)^{-1} \log \frac{\mathscr{P}_{\lambda}(A, B, g[\omega])}{\mathscr{P}_{\lambda}(A, B, g[0])}=-\frac{\lambda}{4} \log \frac{\int e^{4 \omega} d x[0]}{v[0]} \\
\quad-\frac{a_{4}(A, B)-\lambda}{3} \log \frac{\oint e^{3 \omega} d y[0]}{\tilde{v}[0]}-(2 l)^{-1} \log \frac{\operatorname{det}\left(A_{B}\right)[\omega]}{\operatorname{det}\left(A_{B}\right)[0]},
\end{aligned}
$$

where $v[0]=\operatorname{vol}(g[0])$ and $\tilde{v}[0]=\operatorname{vol}(\tilde{g}[0])$. 
In the above, recall that $a_{4}(A, B)$ is conformally invariant. A more manageable version of this formula is obtained when we notice that several of its terms are also terms in a formula for $a_{4}(\omega, A, B)$. Indeed, if $\omega, \eta \in C^{\infty}(M)$, (4.1) can be modified to give a formula for $a_{4}(\omega, A, B)[\eta]$ just be replacing each $[\omega]$ by $[\eta]$. To make the formula even more easily applicable, we add the mild assumption that our chosen background metric on $M$ has constant scalar curvature. We immediately have

4.7. Corollary. Suppose $(M,\langle g[0]\rangle)$ and $(A, B)$ are as in Theorem 2.12 , and that $g[0]$ has constant scalar curvature $\tau_{0}$. Let $\omega \in C^{\infty}(M)$. Then

$$
\begin{aligned}
& -(2 l)^{-1} \log \frac{\operatorname{det}\left(A_{B}\right)[\omega]}{\operatorname{det}\left(A_{B}\right)[0]}=a_{4}(\omega, A, B) \\
& +\beta_{2}\left\{\frac{1}{4} \int \omega(P[0] \omega) d x[0]-\frac{1}{2} \oint \omega(\mathscr{S}(\omega) d y)[0]\right. \\
& \left.\quad+\oint\left(\left(\frac{1}{2} Z_{2}-\frac{1}{6} Z_{4}\right)(\omega, \omega) d y\right)[0]\right\} \\
& +\frac{1}{2} \beta_{3}\left\{\int\left\{\left(J^{2} d x\right)[\omega]-\left(J^{2} d x\right)[0]-\frac{1}{3} \tau_{0} \oint \omega_{\mid N}\right\}\right. \\
& +\sigma_{1} \oint\left(\left(-Z_{4}+3 Z_{5}\right)(\omega, \omega) d y\right)[0] \\
& +\sigma_{2} \oint\left(\left(-6 Z_{1}+3 Z_{2}+2 Z_{3}-Z_{4}\right)(\omega, \omega) d y\right)[0] \\
& +\sigma_{3} \oint\left(\left(-9 Z_{2}-3 Z_{4}\right)(\omega, \omega) d y\right)[0] \\
& +c_{3}\left\{\oint\left(\left(-\frac{3}{2} Z_{2}+\frac{1}{2} Z_{4}\right)(\omega, \omega) d y\right)[0]-\oint\left(E_{1}(\omega, \omega, \omega) d y\right)[0]\right\} \\
& +c_{4}\left\{-3 \oint\left(Z_{3}(\omega, \omega) d y\right)[0]+3 \oint\left(E_{2}(\omega, \omega, \omega) d y\right)[0]\right\} .
\end{aligned}
$$

Now recall the functionals $\mathscr{P}_{\lambda}$ of Remark 2.2, which involve the conformal index $a_{4}(A, B)$. It is sometimes useful to express the conformal index in terms of the Euler characteristic of $M$. Recall that $\chi(M)=\chi(\partial M)+\chi(M, \partial M)$; thus if $m$ is even, $\chi(M)=\chi(M, \partial M)$. By the Chern-Gauss-Bonnet formula, if $m=4$,

$$
\begin{aligned}
\chi(M)= & \left(32 \pi^{2}\right)^{-1} \int_{M}\left(\tau^{2}-4|\rho|^{2}+|R|^{2}\right) d x \\
& +\left(24 \pi^{2}\right)^{-1} \oint_{\partial M}\left(3 \tau H-6 F H-6\langle T, L\rangle+2 H^{3}-6 H|L|^{2}+4 \operatorname{tr} L^{3}\right) d y .
\end{aligned}
$$

The interior integrand can be rewritten as

$$
|C|^{2}-8|V|^{2}+8 J^{2}=|C|^{2}+4\{Q-\Delta J\},
$$

and thus the interior term in (4.3) equals 


$$
\left(32 \pi^{2}\right)^{-1}\left(\int_{M}\left(|C|^{2}+4 Q\right) d x-\frac{2}{3} \oint_{\partial M} \tau_{\mid N} d y\right) \text {. }
$$

Thus

$$
\begin{aligned}
\chi(M)= & \left(32 \pi^{2}\right)^{-1} \int_{M}\left(|C|^{2}+4 Q\right) d x \\
& +\left(24 \pi^{2}\right)^{-1} \oint_{\partial M}\left(-\frac{1}{2} \tau_{\mid N}+3 \tau H-6 F H-6\langle T, L\rangle\right. \\
& \left.+2 H^{3}-6 H|L|^{2}+4 \operatorname{tr} L^{3}\right) d y
\end{aligned}
$$

or more compactly,

$$
\chi(M)=\left(32 \pi^{2}\right)^{-1} \int_{M}\left(|C|^{2}+4 Q\right) d x+\left(4 \pi^{2}\right)^{-1} \oint_{\partial M}\left(S-\mathscr{L}_{4}-\mathscr{L}_{5}\right) d y
$$

At a background metric $g[0]$ with constant scalar curvature as in Corollary 4.7, the $\tau_{\mid N}$ contributions to the boundary integrals in the formulas for $\chi(M)$ and $a_{4}(A, B)$ disappear. It is now appropriate to distinguish two types of "model backgrounds":

4.8. Definition. $(M, g[0])$ is a model background of type $\mathrm{I}$ if $(\nabla R)[0]=0$, $\partial M$ is totally geodesic, and $M$ is connected. $(M, g[0])$ is a model background of type II if $g[0]$ is flat, $(\tilde{\nabla} L)[0]=0$, and $\partial M$ is connected.

4.9. Lemma. In a model background ( $M, g[0])$ of type I, the boundary integrals in (3.4) and (4.4) vanish, and

$$
a_{4}(A[0], B[0])=4 \pi^{2} \beta_{2} \chi(M)+\left(\beta_{1}-\frac{1}{8} \beta_{2}\right)|C|^{2}[0] v[0]
$$

In a model background (M, $g[0])$ of type II, the interior integrals in (3.4) and (4.4) vanish, and

$$
a_{4}(A[0], B[0])=4 \pi^{2} \beta_{2} \chi(M)+\left(\beta_{4}+6 \beta_{2}\right) \mathscr{L}_{4}[0] \tilde{v}[0]+\left(\beta_{5}+6 \beta_{2}\right) \mathscr{L}_{5}[0] \tilde{v}[0] .
$$

Proof. In the type I case, $\nabla R=0 \Rightarrow \nabla C=0$, so the connectedness of $M$ guarantees that $|C|^{2}[0]$ is constant. In the type II case, $R=0, \tilde{\nabla} L=0 \Rightarrow$ $\tilde{\nabla} \mathscr{L}_{4}=\tilde{\nabla} \mathscr{L}_{5}=0$, so the connectedness of $\partial M$ guarantees that $\mathscr{L}_{4}[0]$ and $\mathscr{L}_{5}[0]$ are constant.

We combine these considerations to get a more natural form of (4.2):

4.10. Theorem. Under the assumptions of Theorem 2.12, if $(M, g[0])$ is a background of type I and $\lambda=a_{4}(A, B)$, or if $(M, g[0])$ is a background of type II and $\lambda=0$, 


$$
\begin{aligned}
-(2 l)^{-1} \log \frac{\mathscr{P}_{\lambda}(A, B, g[\omega])}{\mathscr{P}_{\lambda}(A, B, g[0])}=\mathscr{E}_{(\mathrm{I} \text { or II })} & \\
+ & \beta_{2}\left\{\frac{1}{4} \int \omega(P[0] \omega) d x[0]-\frac{1}{3} \oint\left(Y_{3}(\omega) d y\right)[0]\right. \\
& \left.\quad-\frac{1}{2} \oint \omega(\mathscr{S}(\omega) d y)[0]+\oint\left(\left(\frac{1}{2} Z_{2}-\frac{1}{6} Z_{4}\right)(\omega, \omega) d y\right)[0]\right\} \\
+ & \frac{1}{2} \beta_{3} \int\left\{\left(J^{2} d x\right)[\omega]-\left(J^{2} d x\right)[0]\right\}+\sum_{i=1}^{3} \lambda_{i} \oint\left(l_{i}(\omega) d y\right)[0] \\
+ & \sigma_{1} \oint\left(\left(-Z_{4}+3 Z_{5}\right)(\omega, \omega) d y\right)[0] \\
+ & \sigma_{2}\left\{\oint\left(q_{2}(\omega) d y\right)[0]+\oint\left(\left(-6 Z_{1}+3 Z_{2}+2 Z_{3}-Z_{4}\right)(\omega, \omega) d y\right)[0]\right\} \\
+ & \sigma_{3}\left\{\oint\left(q_{3}(\omega) d y\right)[0]+\oint\left(\left(-9 Z_{2}-3 Z_{4}\right)(\omega, \omega) d y\right)[0]\right\} \\
+ & c_{3}\left\{\oint\left(Y_{3}(\omega) d y\right)[0]+\oint\left(\left(-\frac{3}{2} Z_{2}+\frac{1}{2} Z_{4}\right)(\omega, \omega) d y\right)[0]\right. \\
+ & -c_{4}\left\{\oint\left(Y_{4}(\omega) d y\right)[0]-3 \oint\left(Z_{3}(\omega, \omega) d y\right)[0]+3 \oint\left(E_{2}(\omega, \omega, \omega) d y\right)[0]\right\},
\end{aligned}
$$

where

$$
\begin{aligned}
\mathscr{E}_{\mathrm{I}} & =-\frac{a_{4}(A, B)}{4} \log \frac{\int e^{4(\omega-\bar{\omega})} d x[0]}{v[0]} \\
& =-\left\{\pi^{2} \beta_{2} \chi(M)+\frac{1}{4}\left(\beta_{1}-\frac{1}{8} \beta_{2}\right)|C|^{2}[0] v[0]\right\},
\end{aligned}
$$

and

$$
\begin{aligned}
\mathscr{E}_{\text {II }} & =-\frac{a_{4}(A, B)}{3} \log \frac{\oint e^{3(\omega-\tilde{\omega})} d y[0]}{\tilde{v}[0]} \\
& =-\left\{\frac{4}{3} \pi^{2} \beta_{2} \chi(M)+\frac{1}{3}\left(\beta_{4}+6 \beta_{2}\right) \mathscr{L}_{4}[0] \tilde{v}[0]+\frac{1}{3}\left(\beta_{5}+6 \beta_{2}\right) \mathscr{L}_{5}[0] \tilde{v}[0]\right\} .
\end{aligned}
$$

Here $\bar{\omega}:=\left(\int \omega d x[0]\right) / v[0]$ is the mean value of $\omega$ over $M$, and $\tilde{\omega}:=$ $(\oint \omega d y[0]) / \tilde{v}[0]$ is the mean value over $\partial M$.

Proof. In the type I case, we absorb terms totalling

$$
\left(\beta_{1}|C|^{2}[0]+\frac{1}{2} \beta_{2} Q[0]\right) \int \omega d x[0]
$$

(or $\left(\beta_{1,+}\left|C_{+}\right|^{2}[0]+\beta_{1,-}\left|C_{-}\right|^{2}[0]+\frac{1}{2} \beta_{2} Q[0]\right) \int \omega d x[0]$ if $(A, B)$ is orientationsensitive) into the first exponential term of (4.2); the coefficient of the second exponential term is 0 . In the type II case, we absorb terms totalling $\left(\beta_{2} S[0]+\beta_{4} \mathscr{L}_{4}[0]+\beta_{5} \mathscr{L}_{5}[0]\right) \oint \omega d y[0]$ into the second exponential term of (4.2); the coefficient of the first exponential term in 0 . We also make use of the 
fact that $\oint\left(q_{1}(\omega) d y\right)[0]$ vanishes, since $(\tilde{\nabla} L)[0]=0$ in both the type $\mathrm{I}$ and type II cases.

4.11. Remark. The choice $\lambda=a_{4}(A, B)$ or $\lambda=0$ makes $\mathscr{P}_{\lambda}(A, B, g)$ a spectral invariant (recall Remark 2.2). The presence of the $|C|^{2}$ term in the case of a model background of type $I$ is an indication that the analysis of the determinant functional will be heavily dependent on conformal geometry as well as on topology. See [BCY] for this analysis, and the effect of the $|C|^{2}$ term, in the boundariless case. Similarly, for a model background of type II, the $\mathscr{L}_{4}$ and $\mathscr{L}_{5}$ terms indicate a dependence on conformal geometry.

4.12. Remark. There need not be a model background of type I or II in a given conformal class, of course. It can happen, however, that there are model backgrounds of both types in the same conformal class. For example, the round metric on the closed hemisphere $H^{4}$ (type I) is conformal to the flat metric on the closed ball $B^{4}$ (type II). The standard metric on the cylinder $\mathscr{C}_{h}^{4}=$ $[0, h] \times S^{3}$ of height $h$ is conformal to the flat metric on the spherical shell $\mathscr{A}_{s}^{4}=\left\{x \in \mathbb{R}^{4}|1 \leq| x \mid \leq s\right\}, s=e^{h}$. Here the cylindrical geometry is type $\mathrm{I}$; the shell geometry fails to be type II only because of its disconnected boundary.

\section{SPECIAL MANIFOLdS}

We would now like to do some computations in the special cases of the hemisphere, ball, cylinder, and spherical shell. Since the hemisphere and ball are conformally equivalent, and the cylinder of height $h$ is conformally equivalent to the spherical shell of outer/inner radius ratio $s=e^{h}$, it is sufficient to consider the hemisphere and cylinder. Moreover, since we shall later write down the spectra of the Dirichlet and Robin problems for the conformal Laplacian on the hemisphere, and compute the determinants of these problems explicitly (§7), we shall be able to compute the determinants of the similar problems on the ball. The following elementary observation will be useful.

5.1. Lemma. If $\partial M$ is totally geodesic and $\kappa$ is the intrinsic curvature of $\partial M$, then $\tau=\kappa+2 F$ on $\partial M$.

Proof. We use total geodesy to pick coordinates at a point of $\partial M$ which are normal for both $g$ and $\tilde{g}$, then use the characterization of the Riemann tensor as the second-order part of the Taylor expansion of the metric, to show that $\kappa=R_{a b}^{a b}$. Since $\tau=R^{i j}{ }_{i j}=R_{a b}^{a b}+2 R^{a}{ }_{N a N}$, the result follows.

Now consider the upper hemisphere $H^{m}$ in $S^{m}$, the boundary of which is the equator $S^{m-1}$, with the standard round metric $g[0]$ as background. Here the interior metric has $\nabla R=0$ and the boundary embedding is totally geodesic, so $\left(H^{m}, g[0]\right)$ is a model background of type I. In this background, $C=0, V=g / 2$, and $J=m / 2$. If $\Psi^{2}:=\Delta+(m-1)^{2} / 4$, then the Paneitz operator and quantity are

$$
P=\left(\Psi-\frac{3}{2}\right)\left(\Psi-\frac{1}{2}\right)\left(\Psi+\frac{1}{2}\right)\left(\Psi+\frac{3}{2}\right), \quad Q=m(m+2)(m-2) / 8 .
$$

In particular, if $m=4$, then $P=\Delta(\Delta+2)$ and $Q=6$. By (1.1),

$$
R_{i j k l}=-g_{j k} g_{i l}+g_{j l} g_{i k} \text {. }
$$


Thus on the boundary $\partial H^{m}=S^{m-1}$ of $H^{m}$,

$$
G=\tilde{g}, \quad F=m-1, \quad T=(m-2) \tilde{g}
$$

$L$ and $H$ vanish, and by Lemma 3.18, if $f \in C^{\infty}\left(H^{m}\right)$,

$$
Y_{8}(f)=N(-\Delta) f=N^{3} f+(-\tilde{\Delta})(N f)-(m-1) N f .
$$

We also have

$$
\begin{gathered}
X_{i}=0, \quad i=1, \ldots, 8 ; \\
Y_{2}(f)=Y_{3}(f)=Y_{4}(f)=Y_{6}(f)=Y_{7}(f) \\
=Z_{3}(f, f)=Z_{4}(f, f)=Z_{5}(f, f)=0 ;
\end{gathered}
$$

and

$$
Y_{1}(f)=m(m-1) N f, \quad Y_{5}(f)=(m-1) N f .
$$

As a result, on $\partial H^{m}$,

$$
\begin{gathered}
\mathscr{L}_{4}=\mathscr{L}_{5}=0, \\
l_{1}(f)=0, \quad l_{2}(f)=-(m-1) N f, \\
l_{3}(f)=-(m-4)(m-1) N f+N(-\Delta) f \\
=N^{3} f+(-\tilde{\Delta})(N f)-(m-3)(m-1) N f, \\
q_{1}(f)=0, \quad q_{2}(f)=m(m-1)(m-3) N f, \quad q_{3}(f)=m(m-1)^{2} N f .
\end{gathered}
$$

On $\partial H^{4}$,

$$
S=0, \quad \mathscr{S}(f)=N f-\frac{1}{2} N(-\Delta) f=-\frac{1}{2} N^{3} f-\frac{1}{2}(-\tilde{\Delta})(N f)+\frac{5}{2} N f .
$$

Since $\operatorname{vol}\left(H^{4}\right)=4 \pi^{2} / 3$

$$
a_{4}(A, B)=2 \pi^{2} \beta_{2} Q[0] / 3=4 \pi^{2} \beta_{2}
$$

for $(A, B)$ satisfying $1.2,1.6$, and 2.4. (Alternatively, we can use the formula of Lemma 4.9 and the fact that $\chi\left(H^{m}\right)=1$.) Specializing Theorem 4.10, the conclusion is:

5.2. Theorem. Suppose that $(A, B)$ satisfies $1.2,1.6$, and 2.4 , and that $\mathscr{N}\left(A_{B}\right)$ $=0$ on $\left(H^{4}, g[0]\right)$, where $g[0]$ is the round metric. Let $\lambda=4 \pi^{2} \beta_{2}$. Then for 
$\omega \in C^{\infty}\left(H^{4}\right)$,

$$
\begin{aligned}
& -(2 l)^{-1} \log \frac{\mathscr{P}_{\lambda}(A, B, g[\omega])}{\mathscr{P}_{\lambda}(A, B, g[0])}=-\pi^{2} \beta_{2} \log \frac{\int_{H^{4}} e^{4(\omega-\bar{\omega})} d x[0]}{4 \pi^{2} / 3} \\
& +\left(\frac{3}{4} \beta_{2}+3 \sigma_{2}-9 \sigma_{3}-\frac{3}{2} c_{3}\right) \oint_{\partial H^{4}}((N \omega)(-\tilde{\Delta} \omega) d y)[0] \\
& +\beta_{2}\left\{\frac{1}{4} \int_{H^{4}} \omega((\Delta(\Delta+2) \omega) d x)[0]-\frac{5}{4} \oint_{\partial H^{4}} \omega((N \omega) d y)[0]\right. \\
& \left.+\frac{1}{4} \oint_{\partial H^{4}} \omega\left(\left(N^{3} \omega\right) d y\right)[0]\right\} \\
& +\left(-3 \lambda_{2}-3 \lambda_{3}+12 \sigma_{2}+36 \sigma_{3}\right) \oint_{\partial H^{4}}((N \omega) d y)[0] \\
& +\int_{3} \oint_{\partial H^{4}}\left(\left(J^{2} d x\right)[\omega]-\frac{16 \pi^{2}}{3}\right\} \\
& +c_{3} \oint_{\partial H^{4}}\left(\left(N \omega|\tilde{d} \omega|^{2} d y\right)[0]+3 c_{4} \oint_{\partial H^{4}}\left((N \omega)^{3} d y\right)[0] .\right.
\end{aligned}
$$

$A$ formula for the determinant functional $-(2 l)^{-1} \log \left(\operatorname{det}\left(A_{B}\right)[\omega] / \operatorname{det}\left(A_{B}\right)[0]\right)$ is obtained by replacing $-\pi^{2} \beta_{2} \log \left\{\left(\int_{H^{4}} e^{4(\omega-\bar{\omega})} d x[0]\right) /\left(4 \pi^{2} / 3\right)\right\}$ by $4 \pi^{2} \beta_{2} \bar{\omega}$ in the formula above.

To set up a conformal diffeomorphism between the hemisphere and the ball, view $S^{m}$ as the unit sphere of $\mathbb{R}^{m+1}$ with coordinate function $\xi=(u, s) \in$ $\mathbb{R}^{m} \times \mathbb{R}$. Identify $\mathbb{R}^{m}$, whose coordinate will be called $x$, with the complement $S^{m} \backslash(0,-1)$ of the south pole via

$$
x=\frac{u}{1+s}, \quad u=\frac{2 x}{1+r^{2}}, \quad s=\frac{1-r^{2}}{1+r^{2}}=\cos p, \quad \alpha=|u|=\sin p,
$$

where $r=|x|$, and $p$ is the azimuthal angle between the vector $(u, s)$ and the ray emanating from the origin $(0,0)$ and passing through the North Pole $(0,1)$. The standard metrics are related by

$$
g_{\mathbf{R}^{m}}=\Phi^{2} g_{S^{m}}, \quad \Phi=\frac{1}{2}\left(1+r^{2}\right)=1 /(1+s) .
$$

This version of the stereographic projection identifies the upper hemisphere in $S^{m}$ with the unit ball in $R^{m}$; our two conformal metrics agree on the common boundary of $H^{m}$ and $B^{m}$. The total interior volumes of our models differ, as

$$
\operatorname{vol}\left(H^{m}\right)=\frac{(4 \pi)^{m / 2} \Gamma(m / 2)}{2 \Gamma(m)}, \quad \operatorname{vol}\left(B^{m}\right)=\frac{2 \pi^{m / 2}}{m \Gamma(m / 2)},
$$

so that

$$
\frac{\operatorname{vol}\left(B^{m}\right)}{\operatorname{vol}\left(H^{m}\right)}=\frac{\Gamma(m)}{2^{m-2} m \Gamma(m / 2)^{2}}
$$

in particular,

$$
\operatorname{vol}\left(B^{4}\right) / \operatorname{vol}\left(H^{4}\right)=3 / 8
$$


The inward unit normal in $H^{m}$ is $N=-\partial_{p}$. Here we use $p$, together with any coordinate system on the latitudes $s=$ const, to get local coordinates on $H^{m}$. Since $N s=-\partial_{p} s=\alpha$ and $\partial_{s} \alpha=-s / \alpha$,

$$
N=\alpha \partial_{s}, \quad N^{2}=\alpha^{2} \partial_{s}^{2}-s \partial_{s}, \quad N^{3}=\alpha^{3} \partial_{s}^{3}-3 \alpha s \partial_{s}^{2}-\alpha \partial_{s}
$$

on $H^{m} \backslash\{(0,1)\}$. In particular,

$$
N=\partial_{s}, \quad N^{2}=\partial_{s}^{2}, \quad N^{3}=\partial_{s}^{3}-\partial_{s} \quad \text { on } \partial H^{m}
$$

In the notation of Theorem 5.2, if $g[0]$ is the hemisphere metric and $g[\omega]$ the ball metric, then $\omega=-\log (1+s)$. In particular,

$$
\begin{gathered}
\tilde{d} \omega=0, \quad \tilde{\Delta} \omega=0 \quad \text { on } H^{m} \backslash\{(0,1)\}, \\
\omega=0, \quad-N \omega=N^{2} \omega=-N^{3} \omega=1 \quad \text { on } \partial H^{m} .
\end{gathered}
$$

Specializing to the case $m=4$ now, and looking at the formula of Theorem 5.2 , our first need is for $(\Delta(\Delta+2))[0] \omega=P[0] \omega$. Applying our covariant setup in the form (1.7), we can immediately conclude that

$$
P[0] \omega=Q[\omega] e^{4 \omega}-Q[0]=-Q[0]=-6,
$$

since all local scalar invariants vanish in the flat metric $g[\omega] . \quad J^{2}[\omega]$ also vanishes for this reason. The surviving boundary terms in Theorem 5.2 all come from the expressions

$$
\begin{aligned}
\oint_{\partial H^{4}}((N \omega) d y)[0] & =\oint_{\partial H^{4}}\left(\left(N^{3} \omega\right) d y\right)[0]=\oint_{\partial H^{4}}\left((N \omega)\left(N^{2} \omega\right) d y\right)[0] \\
& =\oint_{\partial H^{4}}\left((N \omega)^{3} d y\right)[0]=-\operatorname{vol}\left(\partial H^{4}\right)=-\operatorname{vol}\left(S^{3}\right)=-2 \pi^{2}
\end{aligned}
$$

(Note especially that no $\beta_{2}$ boundary terms survive, since $\omega$ vanishes on the boundary.) Thus Theorem 5.2 specializes to

$$
\begin{gathered}
-(2 l)^{-1} \log \frac{\mathscr{P}_{\lambda}(A, B, g[\omega])}{\mathscr{P}_{\lambda}(A, B, g[0])}=-\pi^{2} \beta_{2}\left\{\log \frac{\operatorname{vol}\left(B^{4}\right)}{4 \pi^{2} / 3}-4 \bar{\omega}\right\}-\frac{3}{2} \beta_{2} \int_{H^{4}} \omega d x[0] \\
-\frac{8}{3} \pi^{2} \beta_{3}-2 \pi^{2}\left(-3 \lambda_{3}-2 \lambda_{3}+6 \sigma_{2}+36 \sigma_{3}+3 c_{4}\right) .
\end{gathered}
$$

In this, the $\bar{\omega}$ and $\int \omega$ terms combine to give

$$
\begin{gathered}
-(2 l)^{-1} \log \frac{\mathscr{P}_{\lambda}(A, B, g[\omega])}{\mathscr{P}_{\lambda}(A, B, g[0])}=-\pi^{2} \beta_{2} \log \frac{\operatorname{vol}\left(B^{4}\right)}{4 \pi^{2} / 3}+\frac{3}{2} \beta_{2} \int_{H^{4}} \omega d x[0] \\
-\frac{8}{3} \beta_{3} \pi^{2}-2 \pi^{2}\left(-3 \lambda_{2}-2 \lambda_{3}+6 \sigma_{2}+36 \sigma_{3}+3 c_{4}\right) .
\end{gathered}
$$

To compute the $\int \omega$ term, let $d \theta$ be a volume form on a standard (radius 1) $S^{3}$; then $\alpha^{3} d \theta$ is a volume form on the latitude in $H^{4}$ with radius $\alpha$, and

$$
-\alpha^{3} d p \wedge d \theta=\alpha^{2} d s \wedge d \theta=\left(1-s^{2}\right) d s \wedge d \theta
$$

is a volume form on $H^{4} \backslash\{(0,1)\}$. Since $\operatorname{vol}\left(S^{3}\right)=2 \pi^{2}$ and $\omega=\omega(s)=$ $-\log (1+s)$ is constant on latitudes,

$$
\begin{aligned}
\int_{H^{4}} \omega d x[0] & =2 \pi^{2} \int_{0}^{1}\left(1-s^{2}\right) \omega(s) d s=-2 \pi^{2} \int_{1}^{2} t(2-t) \log t d t \\
& =\pi^{2}\left(\frac{13}{9}-\frac{8}{3} \log 2\right) .
\end{aligned}
$$


Here we have used the integral formula

$$
\int t^{n}(\log t) d t=\frac{t^{n+1}}{n+1}\left(\log t-\frac{1}{n+1}\right)+\text { const }
$$

in the case $n=1,2$. Since $\operatorname{vol}\left(B^{4}\right)=\pi^{2} / 2$, we have

5.3. Corollary. With assumptions as in Theorem 5.2 and $g[\omega]$ the flat $B^{4}$ metric,

$$
\begin{aligned}
& -(2 l)^{-1} \log \frac{\mathscr{P}_{\lambda}(A, B, g[\omega])}{\mathscr{P}_{\lambda}(A, B, g[0])} \\
& \quad=\pi^{2}\left\{\left(\frac{13}{6}-\log 6\right) \beta_{2}-\frac{8}{3} \beta_{3}+6 \lambda_{2}+4 \lambda_{3}-12 \sigma_{2}-72 \sigma_{3}-6 c_{4}\right\} .
\end{aligned}
$$

The expression for the quotient of determinants (as opposed to scale-invariant determinant functionals) is simply missing the $\operatorname{vol}\left(B^{4}\right) / \operatorname{vol}\left(H^{4}\right)$ contribution: 5.4. Corollary. With assumptions as in Theorem 5.2 and $g[\omega]$ the flat $B^{4}$ metric,

$$
\begin{aligned}
-(2 l)^{-1} \log \frac{\operatorname{det}\left(A_{B}\right)[\omega]}{\operatorname{det}\left(A_{B}\right)[0]} & \\
& =\pi^{2}\left\{\left(\frac{13}{6}-4 \log 2\right) \beta_{2}-\frac{8}{3} \beta_{3}+6 \lambda_{2}+4 \lambda_{3}-12 \sigma_{2}-72 \sigma_{3}-6 c_{4}\right\} .
\end{aligned}
$$

An interesting check on our calculations can be made by specializing Theorem 4.10 to the flat metric on the unit ball $B^{4}$, a model background of type II, and viewing the round $\mathrm{H}^{4}$ metric as the perturbation rather than the background. This is not simply the same calculation in disguise; different terms from the determinant quotient formula contribute to the answer, which is, of course, the reciprocal of the determinant quotient just computed. We omit the details.

Now let $g[0]$ be the standard metric on the cylinder $\mathscr{C}_{h}^{m}=[0, h] \times S^{3}$, that being $g[0]=d t^{2}+d \theta^{2}$, where $t$ is the parameter on $[0, h]$, and $d \theta^{2}$ is the round metric on $S^{m-1}$; this gives a model background of type I. Computing for the moment in a general dimension $m \geq 3$, we have

$$
J=\frac{m-2}{2}, \quad V=\frac{-d t^{2}+d \theta^{2}}{2}, \quad C=0, \quad Q=\frac{m^{2}(m-4)}{8} .
$$

The Paneitz operator is

$$
P=\Delta^{2}+\frac{m(m-4)}{2} \Delta+4 \partial_{t}^{2}+\frac{m^{2}(m-4)^{2}}{16} .
$$

Since $L=0$ and $\tau$ is constant, $X_{i}=0$ for $i=1, \ldots, 8$; in particular, $\mathscr{L}_{4}=$ $\mathscr{L}_{5}=0$, and if $m=4, S=0$. We have $F=0$, and for all $f \in C^{\infty}\left(\mathscr{C}_{h}^{m}\right)$,

$$
\begin{aligned}
& Y_{2}(f)=Y_{3}(f)=Y_{4}(f)=Y_{5}(f)=Y_{6}(f)=Y_{7}(f)=0, \\
& Y_{1}(f)=(m-1)(m-2) N f, \\
& Y_{8}(f)=N^{3} f+(-\tilde{\Delta})(N f) .
\end{aligned}
$$

As a result,

$$
\begin{aligned}
& l_{1}(f)=q_{1}(f)=0, \quad l_{2}(f)=-(m-1)(m-2) N f, \\
& l_{3}(f)=N^{3} f+(-\tilde{\Delta})(N f), \\
& q_{2}(f)=(m-1)(m-2)(m-3) N f, \quad q_{3}(f)=(m-1)^{2}(m-2) N f,
\end{aligned}
$$


and if $m=4$,

$$
\mathscr{S}(f)=2 N f-\frac{1}{2} N^{3} f-\frac{1}{2}(-\tilde{\Delta})(N f) .
$$

Furthermore,

$$
Z_{3}(f, f)=Z_{4}(f, f)=Z_{5}(f, f)=0
$$

Now specialize to the case $m=4$. The conformal index vanishes by the above and Lemma 4.9, since $\chi\left(\mathscr{C}_{h}^{m}\right)=0$; thus the scale-invariant functional involved in the specialization of Theorem 4.10 is $\mathscr{P}_{0}$. Collecting information, we have

5.5. Theorem. Suppose that $(A, B)$ satisfies $1.2,1.6$, and 2.4 , and that $\mathscr{N}\left(A_{B}\right)$ $=0$ on $\left(\mathscr{C}_{h}^{4}, g[0]\right)$, where $g[0]$ is the standard cylinder metric. Let $\mathscr{Y}=-\partial_{t}$. Then for $\omega \in C^{\infty}\left(\mathscr{C}_{h}^{4}\right)$,

$$
\begin{aligned}
&-(2 l)^{-1} \log \frac{\mathscr{P}(A, B, g[\omega])}{\mathscr{P}(A, B, g[0])}=-(2 l)^{-1} \log \frac{\operatorname{det}\left(A_{B}\right)[\omega]}{\operatorname{det}\left(A_{B}\right)[0]} \\
&=\beta_{2}\left\{\frac{1}{4} \int_{\mathscr{C}_{h}^{4}} \omega\left(\left(\Delta^{2}[0]+4 \partial_{t}^{2}\right)\right) \omega d x[0]\right. \\
&\left.\quad-\frac{1}{2} \oint^{*} \omega\left(\left(2 \mathscr{Y} \omega-\frac{1}{2} \mathscr{Y}^{3} \omega-\frac{1}{2}(-\tilde{\Delta})(\mathscr{Y} \omega)\right) d y\right)[0]\right\} \\
&+\frac{1}{2} \beta_{3}\left(\int_{\mathscr{C}_{h}^{4}}\left(J^{2} d x\right)[\omega]-h \operatorname{vol}\left(S^{3}\right)\right) \\
&+\left(-6 \lambda_{2}+6 \sigma_{2}+18 \sigma_{3}\right) \oint^{*}(\mathscr{Y} \omega) d y[0] \\
&+\lambda_{3} \oint^{*}\left(\mathscr{Y}^{3} \omega\right) d y[0]+\lambda_{3} \oint^{*}((-\tilde{\Delta})(\mathscr{Y} \omega) d y)[0] \\
&-6 \sigma_{2} \oint^{*}(\mathscr{Y} \omega)\left(\mathscr{Y}^{2} \omega\right) d y[0] \\
&+\left(\frac{1}{2} \beta_{2}+3 \sigma_{2}-9 \sigma_{3}-\frac{3}{2} c_{3}\right) \oint^{*}(\mathscr{Y} \omega)((-\tilde{\Delta} \omega) d y)[0] \\
&+ c_{3} \oint^{*}(\mathscr{Y} \omega)\left(|\tilde{d} \omega|^{2} d y\right)[0]+3 c_{4} \oint^{*}(\mathscr{Y} \omega)^{3} d y[0]
\end{aligned}
$$

where

$$
\oint^{*}=\oint_{t=h}-\oint_{t=0} .
$$

We specialize further to the perturbation which gives the shell $\mathscr{A}_{s}^{4}$ with $s=e^{h}$. In spherical coordinates, the flat metric on $\mathscr{A}_{s}^{4}$ is $d r^{2}+r^{2} d \theta^{2}$. The diffeomorphism $(t, \theta) \mapsto\left(e^{t}, \theta\right)$ from $\mathscr{C}_{h}^{4}$ to $\mathscr{A}_{s}^{4}$ is conformal:

$$
d r^{2}+r^{2} d \theta^{2}=r^{2}\left(d t^{2}+d \theta^{2}\right)=e^{2 t}\left(d t^{2}+d \theta^{2}\right) .
$$

Thus the shell metric is $g[\omega]$ with $\omega=t$. The surviving terms on the right in Theorem 5.5 are

$$
\beta_{2}\left\{-\frac{1}{2} \oint_{t=h} \omega(2 \mathscr{y} \omega) d y[0]\right\}+\frac{1}{2} \beta_{3}\left(-h \operatorname{vol}\left(S^{3}\right)\right)=2 \pi^{2} h\left(\beta_{2}-\frac{1}{2} \beta_{3}\right) .
$$




\section{The Dirichlet aNd RoBin PROBLeMS FOR THE CONFORMAL LAPLACIAN}

The determinant quotient formulas of Theorem 4.6 involve coefficients $\beta_{\nu}(1 \leq \nu \leq 5), \lambda_{i}(i=1,2,3), \sigma_{j}(j=1,2,3), c_{k}(k=3,4)$ that depend only on the universal formula for $(A, B)$, and not on the particular manifold $M$. In this section, we compute these constants for the two boundary value problems described in Examples 2.7-2.8.

The starting point is a formula of Branson and Gilkey [BG] for $a_{4}(A, B)$ for elliptic boundary value problems $(A, B)$ in the case where (1) $A$ is a secondorder differential operator with metric leading symbol on sections of a vector bundle $V$ over $M$, i.e.,

$$
\sigma_{2}(A)(x, \xi)=|\xi|^{2} \operatorname{Id}_{V_{x}}=g^{i j} \xi_{i} \xi_{j} \operatorname{Id}_{V_{x}},
$$

for all $(x, \xi) \in T^{*} M$, and (2) $B$ gives either Dirichlet conditions, or Neumann conditions of the form

$$
\left.\left(\varphi_{\mid N}+S \varphi\right)\right|_{\partial M}=0
$$

where $S$ is a smooth section of End $\left.V\right|_{\partial M}$. For convenience, we state these results in the present notation. There is no restriction on the dimension $m$, and there no assumptions on naturality or the conformal behavior of $(A, B)$.

6.1. Theorem [BG]. Under the above assumptions on $A$, there is a unique connection $\nabla$ on $V$ such that $A=\Delta_{V}-\mathscr{E}$, where $\Delta_{V}=-g^{i j} \nabla_{i} \nabla_{j}$ is the Bochner Laplacian of $\nabla$, and $\mathscr{E}$ is a smooth section of End $V$. If $B$ gives Dirichlet conditions, we write $a_{n}(f, A, \mathscr{D})$ for $a_{n}(f, A, B)$, and have

$$
\begin{array}{r}
360(4 \pi)^{m / 2} a_{4}(f, A, \mathscr{D}) \\
=\int \operatorname{tr}_{V} f\left\{-60 \Delta_{\mathrm{End} V} \mathscr{E}+60 \tau \mathscr{E}+180 \mathscr{E}^{2}+30 \Omega^{i j} \Omega_{i j}\right. \\
\left.-12 \Delta \tau+5 \tau^{2}-2|\rho|^{2}+2|R|^{2}\right\} \\
+\oint \operatorname{tr}_{\left.V\right|_{\partial M}}\left(f \left\{-120 \mathscr{E}_{\mid N}-18 \tau_{\mid N}+120 \mathscr{E} H+20 \tau H-4 F H+12\langle G, L\rangle\right.\right. \\
\left.-4\langle T, L\rangle-24 \tilde{\Delta} H+\frac{40}{21} H^{3}-\frac{88}{7} H|L|^{2}+\frac{320}{21} \operatorname{tr} L^{3}\right\} \\
+f_{\mid N}\left\{-180 \mathscr{E}-30 \tau-\frac{180}{7} H^{2}+\frac{60}{7}|L|^{2}\right\} \\
\left.+24 f_{\mid N N} H+30(\Delta f)_{\mid N}\right),
\end{array}
$$

where $\Omega$ is the curvature of $\nabla$.

The connection on $V$ determines a connection on End $V$, and this is used to form $\Delta_{\operatorname{End} V}$. The invariants $\oint f L_{a b:}{ }^{a b}=\oint\langle\tilde{\nabla} \tilde{\nabla} f, L\rangle, \oint f \Omega^{a}{ }_{N: a}$, and $\oint f_{\mid N} F$, which a priori are eligible to appear in the above formula, do so with coefficient 0 . Note that we have not quite written things in the form (1.9); $(\Delta f)_{\mid N}$ has been used instead of $f_{\mid N N N}$ in our basis of invariants. This turns out to be convenient for most practical purposes; if desired, Lemma 3.18 can be used to switch to the basis used in (1.9). 
6.2. Theorem [BG]. Under the above assumptions, if $B$ gives Neumann conditions of the form (6.1), we write $a_{n}(f, A, S)$ for $a_{n}(f, A, B)$, and have

$$
\begin{aligned}
& 360(4 \pi)^{m / 2} a_{4}(f, A, S) \\
& =\int \operatorname{tr}_{V} f\left\{-60 \Delta_{\text {End } V} \mathscr{E}+60 \tau \mathscr{E}+180 \mathscr{E}^{2}+30 \Omega^{i j} \Omega_{i j}\right. \\
& \left.-12 \Delta \tau+5 \tau^{2}-2|\rho|^{2}+2|R|^{2}\right\} \\
& +\oint \operatorname{tr}_{\left.V\right|_{\partial M}}\left(f \left\{240 \mathscr{E}_{\mid N}+42 \tau_{\mid N}+120 \mathscr{E} H+20 \tau H-4 F H\right.\right. \\
& +12\langle G, L\rangle-4\langle T, L\rangle-24 \tilde{\Delta} H \\
& +\frac{40}{3} H^{3}+8 H|L|^{2}+\frac{32}{3} \operatorname{tr} L^{3}+720 S \mathscr{E}+120 S \tau \\
& \left.+144 S H^{2}+48 S|L|^{2}+480 S^{2} H+480 S^{3}-120 \tilde{\Delta}_{\text {End } \left.\left.V\right|_{\partial M} S\right\}} S\right\} \\
& +f_{\mid N}\left\{180 \mathscr{E}+30 \tau+12 H^{2}+12|L|^{2}+72 S H+240 S^{2}\right\} \\
& \left.+f_{\mid N N}\{24 H+120 S\}-30(\Delta f)_{\mid N}\right) \text {. }
\end{aligned}
$$

Again the invariants $\oint f L_{a b:}{ }^{a b}=\oint\langle\tilde{\nabla} \tilde{\nabla} f, L\rangle, \oint f \Omega^{a}{ }_{N: a}$, and $\oint f_{\mid N} F$ appear with coefficient 0 , as does the new invariant $\oint f S F$.

For the conformal Laplacian $Y$ with either Dirichlet or Robin conditions,

$$
\Omega=0, \quad \mathscr{E}=-\frac{m-2}{2} J=-\frac{m-2}{4(m-1)} \tau .
$$

To evaluate the interior terms of $a_{4}$ for either problem, we compute that $\tau^{2}=$ $4(m-1)^{2} J^{2},|\rho|^{2}=(m-2)^{2}|V|^{2}+(3 m-4) J^{2},|R|^{2}=|C|^{2}+4(m-2)|V|^{2}+4 J^{2}$. Recall the formula (1.5) for $Q$. Writing $(Y, \mathscr{D})$ and $(Y, \mathscr{R})$ for the Dirichlet and Robin problems, we have:

6.3. Lemma. The interior terms of

$$
360(4 \pi)^{m / 2} a_{4}(f, Y, \mathscr{D})
$$

or of

$$
360(4 \pi)^{m / 32} a_{4}(f, Y, \mathscr{R})
$$

in the formula of Theorem 6.1 or 6.2 are

$$
\begin{aligned}
& \int f\left(2|C|^{2}-2(m-2)(m-6)|V|^{2}\right.\left.+(5 m-16)(m-6) J^{2}+6(m-6) \Delta J\right) \\
&=\int f\left(2|C|^{2}+2(m-6) Q-2(m-4)(m-6)|V|^{2}\right. \\
&\left.+4(m-4)(m-6) J^{2}+4(m-6) \Delta J\right) .
\end{aligned}
$$

The local invariants in the last expression are linearly dependent, but those that survive upon restriction to dimension $m=4$ are linearly independent. The factor of $m-6$ in the terms that are not local conformal invariants is expected; see [BG, Lemma 3.1(c)]. Recalling the notation of Tables 3.1 and 3.2, we have: 
6.4. Lemma. The boundary terms of $360(4 \pi)^{m / 2} a_{4}(f, Y, \mathscr{D})$ in the formula of Theorem 6.1 are

$$
\begin{array}{r}
\oint\left(f \left\{\frac{6(2 m-7)}{m-1} X_{1}-\frac{10(m-4)}{m-1} X_{2}-4 X_{3}\right.\right. \\
\left.+12 X_{4}-4 X_{5}+\frac{40}{21} X_{6}-\frac{88}{7} X_{7}+\frac{320}{21} X_{8}\right\} \\
+\frac{15(m-4)}{m-1} Y_{1}(f)+24 Y_{2}(f)+24 Y_{3}(f) \\
\left.\quad-\frac{180}{7} Y_{4}(f)+\frac{60}{7} Y_{7}(f)-30 Y_{8}(f)\right) .
\end{array}
$$

(The invariants $Y_{5}(f)$ and $Y_{6}(f)$ appear with coefficient 0.$)$

We now change the basis of invariants to that of Theorem 3.7, and check that the coefficients $c$ and $\gamma_{i}$ vanish as asserted there. For this, note that in dimension 4,

$$
\begin{aligned}
\mathscr{L}_{4} & =-\frac{1}{3} X_{2}+X_{3}-X_{4}+X_{5}, \\
\mathscr{L}_{5} & =-\frac{2}{9} X_{6}+X_{7}-X_{8}, \\
l_{1}(f) & =\left(-Y_{4}+3 Y_{7}\right)(f), \\
l_{2}(f) & =\left(-Y_{1}-Y_{2}+Y_{3}-\frac{1}{3} Y_{4}+3 Y_{5}\right)(f), \quad(m=4) \\
l_{3}(f) & =\left(\frac{2}{3} Y_{2}-\frac{8}{3} Y_{3}+\frac{2}{9} Y_{4}+2 Y_{6}+Y_{8}\right)(f), \\
q_{1}(f) & =\left(Y_{3}-3 Y_{6}\right)(f), \\
q_{2}(f) & =\left(Y_{1}+2 Y_{2}-4 Y_{3}\right)(f), \\
q_{3}(f) & =\left(3 Y_{1}-6 Y_{2}+4 Y_{4}\right)(f) .
\end{aligned}
$$

We then change to the basis of (4.1) and compute the following.

6.5. Theorem. If $\bar{\beta}_{\nu}=(4 \pi)^{2} \cdot 360 \beta_{\nu}$ and similarly for $\lambda_{i}, \sigma_{j}$, and $c_{k}$, then for the problem $(Y, \mathscr{D})$ in dimension $m=4$, we have $\bar{\beta}_{1}=2, \bar{\beta}_{2}=\bar{\beta}_{3}=-8$, $\bar{\beta}_{4}=-4, \bar{\beta}_{5}=-88 / 7, \bar{\lambda}_{1}=20 / 7, \bar{\lambda}_{2}=0, \bar{\lambda}_{3}=-30, \bar{\sigma}_{1}=-20, \bar{\sigma}_{2}=35 / 3$, $\bar{\sigma}_{3}=-31 / 9, \bar{c}_{3}=8, \bar{c}_{4}=-152 / 63$.

For the Robin problem,

$$
S=-\frac{m-2}{2(m-1)} H
$$

The easiest way to compute is to find the difference between the Robin and 
Dirichlet heat invariants; this we do in dimension $m=4$ only:

$$
\begin{aligned}
(4 \pi)^{2} & \cdot 360\left(a_{4}(f, Y, \mathscr{R})-a_{4}(f, Y, \mathscr{D})\right) \\
=\oint(f & \left\{-\frac{64}{63} H^{3}+\frac{32}{7} H|L|^{2}-\frac{32}{7} \operatorname{tr} L^{3}+40 \tilde{\Delta} H\right\} \\
& \left.\quad+f_{\mid N}\left\{\frac{848}{21} H^{2}+\frac{24}{7}|L|^{2}\right\}-40 f_{\mid N N} H-60(\Delta f)_{\mid N}\right) \\
=\oint\left(f\left\{-\frac{64}{63} X_{6}+\frac{32}{7} X_{7}-\frac{32}{7} X_{8}\right\}-40 Y_{2}(f)-40 Y_{3}(f)\right. & \\
& \left.+\frac{848}{21} Y_{4}(f)+\frac{24}{7} Y_{7}(f)+60 Y_{8}(f)\right)
\end{aligned}
$$

Using (6.2), we write this in terms of the invariants $\mathscr{L}_{\nu}, l_{i}(f), q_{j}(f), Y_{3}(f)$, and $Y_{4}(f)$ :

$$
\begin{aligned}
(4 \pi)^{2} & \cdot 360\left(a_{4}(f, Y, \mathscr{R})-a_{4}(f, Y, \mathscr{D})\right) \\
= & \oint\left(\frac{32}{7} f \mathscr{L}_{5}+\frac{8}{7} l_{1}(f)+60 l_{3}(f)+40 q_{1}(f)\right. \\
& \left.-20 q_{2}(f)+\frac{20}{3} q_{3}(f)+\frac{32}{21} Y_{4}(f)\right), \quad m=4 .
\end{aligned}
$$

This gives

6.6. Theorem. If $\bar{\beta}_{\nu}=(4 \pi)^{2} \cdot 360 \beta_{\nu}$ and similarly for $\lambda_{i}, \sigma_{j}$, and $c_{k}$, then for the problem $(Y, \mathscr{R})$ in dimension $m=4$, we have $\bar{\beta}_{1}=2, \bar{\beta}_{2}=\bar{\beta}_{3}=-8$, $\bar{\beta}_{4}=-4, \bar{\beta}_{5}=-8, \bar{\lambda}_{1}=4, \bar{\lambda}_{2}=0, \bar{\lambda}_{3}=30, \bar{\sigma}_{1}=20, \bar{\sigma}_{2}=-25 / 3$, $\bar{\sigma}_{3}=29 / 9, \bar{c}_{3}=8, \bar{c}_{4}=-8 / 9$.

Apropos Theorems 5.2 and 5.5, we remark that

$$
\begin{aligned}
& \left(\frac{3}{4} \bar{\beta}_{2}+3 \bar{\sigma}_{2}-9 \bar{\sigma}_{3}-\frac{3}{2} \bar{c}_{3}\right)(Y, \mathscr{D})=48, \\
& \left(\frac{3}{4} \bar{\beta}_{2}+3 \bar{\sigma}_{2}-9 \bar{\sigma}_{3}-\frac{3}{2} \bar{c}_{3}\right)(Y, \mathscr{R})=-72, \\
& \left(-3 \bar{\lambda}_{2}-3 \bar{\lambda}_{3}+12 \bar{\sigma}_{2}+36 \bar{\sigma}_{3}\right)(Y, \mathscr{D})=106, \\
& \left(-3 \bar{\lambda}_{2}-3 \bar{\lambda}_{3}+12 \bar{\sigma}_{2}+36 \bar{\sigma}_{3}\right)(Y, \mathscr{R})=-74, \\
& \left(\frac{1}{2} \bar{\beta}_{2}+3 \bar{\sigma}_{2}-9 \bar{\sigma}_{3}-\frac{3}{2} \bar{c}_{3}\right)(Y, \mathscr{D})=50, \\
& \left(\frac{1}{2} \bar{\beta}_{2}+3 \bar{\sigma}_{2}-9 \bar{\sigma}_{3}-\frac{3}{2} \bar{c}_{3}\right)(Y, \mathscr{R})=-70, \\
& \left(-6 \bar{\lambda}_{2}+6 \bar{\sigma}_{2}+18 \bar{\sigma}_{3}\right)(Y, \mathscr{D})=\left(-6 \bar{\lambda}_{2}+6 \bar{\sigma}_{2}+18 \bar{\sigma}_{3}\right)(Y, \mathscr{R})=8 .
\end{aligned}
$$

By Corollaries 5.3 and 5.4 and equation (5.1), we have

6.7. Corollary. If $g[0]$ is the standard $H^{4}$ metric and $g[\omega]$ the standard $B^{4}$ metric, then $a_{4}(Y, \mathscr{D})=a_{4}(Y, \mathscr{R})=-1 / 180$. For $\lambda=-1 / 180$, 


$$
\begin{aligned}
\log \frac{\mathscr{P}_{\lambda}(Y, \mathscr{D}, g[\omega])}{\mathscr{P}_{\lambda}(Y, \mathscr{D}, g[0])} & =\frac{-\left(\log 6+\frac{17}{21}\right)}{360}<0, \\
\log \frac{\operatorname{det}\left(Y_{\mathscr{D}}\right)[\omega]}{\operatorname{det}\left(Y_{\mathscr{D}}\right)[0]} & =\frac{-\left(4 \log 2+\frac{17}{21}\right)^{\circ}}{360}<0, \\
\log \frac{\mathscr{P}_{\lambda}(Y, \mathscr{R}, g[\omega])}{\mathscr{P}_{\lambda}(Y, \mathscr{R}, g[0])} & =\frac{-\left(\log 6-\frac{1}{3}\right)}{360}<0, \\
\log \frac{\operatorname{det}\left(Y_{\mathscr{R}}\right)[\omega]}{\operatorname{det}\left(Y_{\mathscr{R}}\right)[0]} & =\frac{-\left(4 \log 2-\frac{1}{3}\right)}{360}<0 .
\end{aligned}
$$

If $g[0]$ is the standard $\mathscr{C}_{h}^{4}$ metric and $g[\omega]$ the standard $\mathscr{A}_{s}^{4}$ metric, $s=e^{h}$, then $a_{4}(Y, \mathscr{D})=a_{4}(Y, \mathscr{R})=0$, and

$$
\log \frac{\mathscr{P}_{0}(Y, \mathscr{D}, g[\omega])}{\mathscr{P}_{0}(Y, \mathscr{D}, g[0])}=\log \frac{\operatorname{det}\left(Y_{\mathscr{D}}\right)[\omega]}{\operatorname{det}\left(Y_{\mathscr{D}}\right)[0]}=\frac{h}{360}
$$

Proof. Aside from direct computation, what we need to verify is that the null spaces of the problems vanish on the manifolds in question. But the lowest possible eigenvalue of either problem on $H^{4}$ or $\mathscr{C}_{h}^{4}$ is $1 / 6$ times the (positive) constant scalar curvature of $g[0]$.

Branson, Chang, and Yang [BCY, §5] have shown that the scale-invariant determinant functional for $Y$ on the conformal class of the round metric $g$ [0] on $S^{4}$ is minimized exactly at $g[0]$, and at the metrics $h^{*} g[0]$ gotten by pulling $g$ [0] back under a conformal diffeomorphism $h$ of $\left(S^{4}, g[0]\right)$. In light of this, Corollary 6.7 can be interpreted as saying that passage from $H^{4}$ to $B^{4}$ has improved (i.e., lowered) the scale-invariant determinant functionals for both $(Y, \mathscr{D})$ and $(Y, \mathscr{R})$. Roughly speaking, round is "best" in the boundariless case, but flat is "better" when boundaries are allowed.

\section{The VAlue of THE FUnCTIONAL DETERMinANT ON THE HEMISPHERE AND BALL}

In this section, the index $j$ will always run over the natural numbers $\mathbb{N}$.

The Hurwitz zeta functions are

$$
\zeta_{a}(s)=\sum_{j}(j+a)^{-s}, \quad a>0,
$$

and the Riemann zeta function is $\zeta_{R}(s)=\zeta_{1}(s)$. Note that

$$
(d / d a) \zeta_{a}(s)=-s \zeta_{a}(s+1), \quad \zeta_{a}(s)-\zeta_{a+1}(s)=a^{-s} .
$$

Consider the double zeta functions

$$
h_{a}(s)=\sum_{j}[(j+a)(j+a+1)]^{-s}, \quad f_{a}(s)=\sum_{j}(2 j+2 a+1)[(j+a)(j+a+1)]^{-s} \text {. }
$$

In analogy with (7.1), we have

$$
\begin{aligned}
(d / d a) h_{a}(s) & =-s f_{a}(s+1), \\
(d / d a) f_{a}(s) & =(2-4 s) h_{a}(s)-s h_{a}(s+1), \\
h_{a}(s)-h_{a+1}(s) & =[a(a+1)]^{-s}, \\
f_{a}(s)-f_{a+1}(s) & =(2 a+1)[a(a+1)]^{-s} .
\end{aligned}
$$


All these zeta functions have isolated simple poles. All identities below are valid in their elementary form for large $\operatorname{Re} s$, and for all $s$ in the sense of analytic continuation. In particular, a quantity expressed as a sum or product of terms, some of which are singular at a given value of $s$, might still be regular at that $s$.

The Riemann zeta function satisfies

$$
\begin{gathered}
\zeta_{R}(-2 m)=0, \quad \zeta_{R}(1-2 m)=(-1)^{m} B_{m} / 2 m \quad\left(m \in \mathbb{Z}^{+}\right) ; \\
\zeta_{R}(0)=-\frac{1}{2},
\end{gathered}
$$

where the $B_{m}$ are the Bernoulli numbers: $B_{1}=1 / 6, B_{2}=1 / 30, B_{3}=1 / 42$, $B_{4}=1 / 30, B_{5}=5 / 66, \ldots$ [WW, 13.15]. Further [WW, 13.21], $\zeta_{a}(0)=\frac{1}{2}-a$.

A generalization of a calculation in [W, Appendix C] gives, for the double zeta functions,

$$
h_{a}(-m)=\frac{(-1)^{m}(m !)^{2}}{2(2 m+1)^{2}}+\sum_{k=0}^{m}(-1)^{k}\left(\begin{array}{c}
m \\
k
\end{array}\right) \zeta_{a+1}(-2 m+k), \quad m \in \mathbb{N} .
$$

(See the appendix for this calculation.) Differentiating using (7.2), we get

$$
f_{a}(1-m)=\frac{1}{m} \sum_{k=0}^{m}(-1)^{k}\left(\begin{array}{c}
m \\
k
\end{array}\right)(2 m-k) \zeta_{a+1}(-2 m+k+1),
$$

$$
m \in \mathbb{Z}^{+} \text {. }
$$

(It is tedious, but possible, to check the derivative of this formula against (7.3); the values given in (7.4) are necessary for this.) In particular,

$$
\begin{aligned}
& h_{a}(0)=-a, \quad h_{a}(-1)=-\frac{1}{18}+\zeta_{a+1}(-2)-\zeta_{a+1}(-1), \\
& f_{a}(0)=\frac{1}{3}-a^{2}, \quad f_{a}(-1)=2 \zeta_{a+1}(-3)-3 \zeta_{a+1}(-2)+\zeta_{a+1}(-1) .
\end{aligned}
$$

If $a$ is a natural number in the above formulas,

$$
h_{a}(-1)=\frac{1}{36}-\frac{1}{3} a\left(a^{2}-1\right), \quad f_{a}(-1)=-\frac{1}{15}-\frac{1}{2} a^{2}\left(a^{2}-1\right)
$$

by (7.1) and (7.4). (These formulas are actually good for general $a>0$, and follow from (8.2) below.)

Now consider $s$-derivatives, denoted by a prime. In analogy with

$$
\zeta_{a}^{\prime}(0)=\log \Gamma(a)-\frac{1}{2} \log 2 \pi
$$

[WW, 13.21], and again generalizing [W, Appendix C], we have

$$
\begin{aligned}
h_{a}^{\prime}(0) & =2 \zeta_{a+1}^{\prime}(0)-\log a \\
h_{a}^{\prime}(-1) & =2 \zeta_{a+1}^{\prime}(-2)-\left(a^{2}+a\right) \log a, \\
f_{a}^{\prime}(0) & =4 \zeta_{a+1}^{\prime}(-1)-\frac{1}{2}-(2 a+1) \log a, \\
f_{a}^{\prime}(-1) & =4 \zeta_{a+1}^{\prime}(-3)+2 \zeta_{a+1}^{\prime}(-1)-a(a+1)(2 a+1) \log a+\frac{1}{24} .
\end{aligned}
$$

(Again, see the appendix for the calculation.) 
An understanding of the double zeta functions is sufficient to compute the determinant of the conformal Laplacian $Y$, with Dirichlet or Robin conditions, on the hemisphere $H^{m}$ in $S^{m}$, the boundary of which is the equator $S^{m-1}$. By standard theory of spherical harmonics, $Y$ takes the value

$$
\lambda_{m, j}:=\left(j+\frac{m-2}{2}\right)\left(j+\frac{m}{2}\right),
$$

on the space $E_{m, j}$ of spherical harmonics of degree $j$. Since

$$
\operatorname{dim} E_{m, j}=N_{m, j}=\frac{m+2 j-1}{(m-1) !}(m+j-2) \cdots(j+1),
$$

the zeta function of $Y$ is

$$
\begin{aligned}
\zeta_{Y}^{S^{m}}(s)= & \frac{1}{(m-1 !} \sum_{j=0}^{\infty}(m+2 j-1)\{(m+j-2) \cdots(j+1)\} \\
& \times\left\{\left(j+\frac{m-2}{2}\right)\left(j+\frac{m}{2}\right)\right\}^{-s}
\end{aligned}
$$

for $m \geq 3$. Noting that the factors in both $\lambda_{m, j}$ and $N_{m, j}$ exhibit a certain symmetry about the value $j+(m-1) / 2$, we can perform the following trick. If $m$ is even, define a polynomial $B_{m}(x)$ and integers $b_{\alpha, m}$ by

$$
B_{m}(x)=\prod_{p=0}^{(m-4) / 2}(x-p(p+1))=\sum_{\alpha=1}^{(m-2) / 2} b_{\alpha, m} x^{\alpha}
$$

Then

$$
\zeta_{Y}^{S^{m}}(s)=\frac{1}{(m-1) !} \sum_{\alpha=1}^{(m-2) / 2} b_{\alpha, m} f_{(m-2) / 2}(s-\alpha), \quad m \text { even. }
$$

Recall that this really expresses $\zeta_{Y}^{S^{m}}$ in terms of $f_{1}$, since

$$
f_{(m-2) / 2}(s)=f_{1}(s)-\sum_{q=1}^{(m-4) / 2}(2 q+1)\{q(q+1)\}^{-s} .
$$

In fact, the situation is even simpler than this: we can actually replace $f_{(m-2) / 2}$ by $f_{1}$ in $(7.8)$, since

$$
\sum_{\alpha=1}^{(m-2) / 2} b_{\alpha, m}\{q(q+1)\}^{\alpha}=B_{m}(q(q+1))=0
$$

for $q=1,2, \ldots,(m-4) / 2$. The result is

$$
\zeta_{Y}^{S^{m}}(s)=\frac{1}{(m-1) !} \sum_{\alpha=1}^{(m-2) / 2} b_{\alpha, m} f_{1}(s-\alpha), \quad m \text { even. }
$$

The case $m=2$ is exceptional in that the zero eigenvalue must be thrown out before the construction of the zeta function; the result is $\zeta_{\Delta}^{S^{2}}(s)=f_{1}(s)$. Special cases of (7.9) are

$$
\zeta_{\Delta+2}^{S^{4}}(s)=\frac{1}{6} f_{1}(s-1), \quad \zeta_{\Delta+6}^{S^{6}}(s)=\frac{1}{120}\left\{f_{1}(s-2)-2 f_{1}(s-1)\right\}
$$


If $m$ is odd, define a polynomial $C_{m}(x)$ and rational numbers $c_{\alpha, m}$ by

$$
C_{m}(x)=\prod_{p=0}^{(m-3) / 2}\left(x-\left(p+\frac{1}{2}\right)\left(p-\frac{1}{2}\right)\right)=\sum_{\alpha=0}^{(m-1) / 2} c_{\alpha, m} x^{\alpha} .
$$

Then

$$
\zeta_{Y}^{S^{m}}(s)=\frac{2}{(m-1) !} \sum_{\alpha=0}^{(m-1) / 2} c_{\alpha, m} h_{(m-2) / 2}(s-\alpha), \quad m \text { odd. }
$$

This is a formula in terms of $h_{1 / 2}$, since

$$
h_{(m-2) / 2}(s)=h_{1 / 2}(s)-\sum_{q=1}^{(m-3) / 2}\left\{\left(q+\frac{1}{2}\right)\left(q-\frac{1}{2}\right)\right\}^{-s} .
$$

In fact, since

$$
\sum_{\alpha=0}^{(m-1) / 2} c_{\alpha, m}\left\{\left(q+\frac{1}{2}\right)\left(q-\frac{1}{2}\right)\right\}^{\alpha}=C_{m}\left(\left(q+\frac{1}{2}\right)\left(q-\frac{1}{2}\right)\right)=0
$$

for $q=1,2, \ldots,(m-3) / 2$, we may simply replace $h_{(m-2) / 2}$ by $h_{1 / 2}$ :

$$
\zeta_{Y}^{S^{m}}(s)=\frac{2}{(m-1) !} \sum_{\alpha=0}^{(m-1) / 2} c_{\alpha, m} h_{1 / 2}(s-\alpha), \quad m \text { odd. }
$$

As special cases, we get

$$
\begin{aligned}
\zeta_{\Delta+3 / 4}^{S^{3}}(s) & =h_{1 / 2}(s-1)+\frac{1}{4} h_{1 / 2}(s), \\
\zeta_{\Delta+15 / 4}^{S^{4}}(s) & =\frac{1}{12}\left\{h_{1 / 2}(s-2)-\frac{1}{2} h_{1 / 2}(s-1)-\frac{3}{16} h_{1 / 2}(s)\right\} .
\end{aligned}
$$

Now consider the conformally covariant Dirichlet and Robin problems on the hemisphere $H^{m}$ with its standard metric $g[0]$. Since the equator is totally geodesic, the mean curvature vanishes, so that the Robin problem is just the standard Neumann problem. The spectral resolutions of these problems are as follows: Dirichlet eigenfunctions are spherical harmonics on $S^{m}$ which are odd across the equator, while Robin eigenfunctions are those which are even. A standard counting argument from the theory of spherical harmonics shows that the space $E_{j}$ contributes multiplicities $N_{m, j, R}$ and $N_{m, j, D}$ to the Robin and Dirichlet spectra respectively, where

$$
\begin{aligned}
& N_{m, j, R}=\sum_{\substack{0 \leq k \leq j \\
j-k \text { even }}} N_{m-1, k}=\frac{(m+j-1) \cdots(j+1)}{(m-1) !}, \\
& N_{m, j, D}=\sum_{\substack{0 \leq k \leq j \\
j-k \text { odd }}} N_{m-1, k}=\frac{(m+j-2) \cdots j}{(m-1) !} .
\end{aligned}
$$

From a representation-theoretic viewpoint, this can be derived from the branching rule describing the decomposition of $\mathrm{SO}(m)$-modules under restriction to a standard (embedded by block stabilization) $\mathrm{SO}(m-1)$ subgroup, together with 
Weyl's dimension formula. See, e.g., [Br4] for details of both the branching rule and dimension formula. Calculating as above for $m$ even, we get

$\zeta_{Y, \pm}^{H^{m}}(s)=\frac{1}{(m-1) !} \sum_{\alpha=1}^{(m-2) / 2} b_{\alpha, m}\left\{\frac{1}{2} f_{1}(s-\alpha) \pm \frac{m-1}{2} h_{1}(s-\alpha)\right\}, \quad m$ even,

where $\zeta_{Y,+}^{H^{m}}$ is the zeta function of the Robin problem, and $\zeta_{Y,-}^{H^{m}}$ is the zeta function of the Dirichlet problem. If $m$ is odd, we define

$$
\tilde{C}_{m}(x)=\frac{C_{m}(x)}{x+\frac{1}{4}}=\prod_{p=1}^{(m-3) / 2}\left(x-\left(p+\frac{1}{2}\right)\left(p-\frac{1}{2}\right)\right)=\sum_{\beta=0}^{(m-3) / 2} \tilde{c}_{\beta, m} x^{\beta},
$$

and compute that

$\zeta_{Y, \pm}^{H^{m}}(s)=\frac{1}{(m-1) !}\left\{\sum_{\alpha=0}^{(m-1) / 2} c_{\alpha, m} h_{1 / 2}(s-\alpha) \pm \frac{m-1}{4} \sum_{\beta=0}^{(m-3) / 2} \tilde{c}_{\beta, m} f_{1 / 2}(s-\beta)\right\}$,

$m$ odd.

For example, on $H^{2}$, the zeta function of the Robin problem is $\frac{1}{2}\left(f_{1}(s)+h_{1}(s)\right)$, as opposed to $\frac{1}{2}\left(f_{1}(s)-h_{1}(s)\right)$ for the Dirichlet problem. On $H^{4}$, the Robin zeta function is $\frac{1}{12} f_{1}(s-1)+\frac{1}{4} h_{1}(s-1)$, and the Dirichlet zeta function is $\frac{1}{12} f_{1}(s-1)-\frac{1}{4} h_{1}(s-1)$. On $H^{3}$, the Robin zeta function is

$$
\frac{1}{2}\left\{\frac{1}{4} h_{1 / 2}(s)+h_{1 / 2}(s-1)+\frac{1}{2} f_{1 / 2}(s)\right\},
$$

and the Dirichlet zeta function is $\frac{1}{2}\left\{\frac{1}{4} h_{1 / 2}(s)+h_{1 / 2}(s-1)-\frac{1}{2} f_{1 / 2}(s)\right\}$, In particular:

7.1. Theorem. On $\mathrm{H}^{2}, \mathrm{H}^{3}$, and $\mathrm{H}^{4}$ with their standard metrics,

$$
\begin{aligned}
& \left(\zeta_{\Delta, \pm}^{H^{2}}\right)^{\prime}(0)=2 \zeta_{R}^{\prime}(-1)-\frac{1}{4} \pm \zeta_{R}^{\prime}(0), \\
& \left(\zeta_{Y, \pm}^{H^{3}}\right)^{\prime}(0)=-\frac{3}{4} \zeta_{R}^{\prime}(-2)+\frac{1}{4} \log 2 \pm\left(-\frac{1}{2} \zeta_{R}^{\prime}(-1)-\frac{1}{8}-\frac{1}{24} \log 2\right), \\
& \left(\zeta_{Y, \pm}^{H^{4}}\right)^{\prime}(0)=\frac{1}{3} \zeta_{R}^{\prime}(-3)+\frac{1}{6} \zeta_{R}^{\prime}(-1)+\frac{1}{288} \pm \frac{1}{2} \zeta_{R}^{\prime}(-2) .
\end{aligned}
$$

Proof. Besides the above computations, we use (7.7); the fact that $\zeta_{2}(s)=$ $\zeta_{R}(s)-1$, so that $\zeta_{2}^{\prime}(s)=\zeta_{R}^{\prime}(s)$; and the identity

$$
\zeta_{a+1 / 2}(s)=2^{s} \zeta_{2 a}(s)-\zeta_{a}(s)
$$

in the case $a=1$.

Theorem 7.1 gives the value of $-\log \operatorname{det} Y_{ \pm}$on $H^{4}$. By virtue of Corollary 6.7, we can also give the value of these functionals on $B^{4}$ :

7.2. Corollary. On $B^{4}$ with its standard metric,

$-\log \operatorname{det} Y_{-}=\frac{1}{3} \zeta_{R}^{\prime}(-3)+\frac{1}{6} \zeta_{R}^{\prime}(-1)+\frac{1}{288}-\frac{1}{2} \zeta_{R}^{\prime}(-2)+\left(4 \log 2+\frac{17}{21}\right) / 360$, 


$$
-\log \operatorname{det} Y_{+}=\frac{1}{3} \zeta_{R}^{\prime}(-3)+\frac{1}{6} \zeta_{R}^{\prime}(-1)+\frac{1}{288}+\frac{1}{2} \zeta_{R}^{\prime}(-2)+\left(4 \log 2-\frac{1}{3}\right) / 360 \text {. }
$$

\section{APPENDIX}

a. Local invariants. Here, for ease of reference, we record the formulas from $\S \S 1$ and 3 which define the local invariants used in our computations. We refer to the beginnings of those sections for conventions on the use of the invariant index notation. In particular, we shall not bother to use raised indices here; when an index occurs twice in an expression, one copy should be raised before summing. Our sign conventions for the Riemann curvature tensor and Laplacian are

$$
R_{1212}>0 \text { on standard spheres, } \Delta=-(d / d x)^{2} \text { on } \mathbb{R}^{1} \text {. }
$$

The basic curvature and fundamental form quantities are

$$
\begin{gathered}
\rho_{i j}=R_{k i k j}, \quad \tau=\rho_{i i}, \quad L_{a b}=-\frac{1}{2} N g_{a b}, \quad H=L_{a a}, \\
G_{a b}=R_{a N b N}, \quad F=G_{a a}, \quad T_{a b}=R_{c a c b} .
\end{gathered}
$$

Note that $T_{a a}=\tau-2 F . m$ always denotes the dimension of (the interior of) the underlying manifold $(M, \partial M)$. The quantities

$$
\begin{gathered}
J=\tau / 2(m-1), \quad V=(\rho-J g) /(m-2), \\
C_{i j k l}=R_{i j k l}+V_{j k} g_{i l}-V_{j l} g_{i k}+V_{i l} g_{j k}-V_{i k} g_{j l},
\end{gathered}
$$

are sometimes better adapted to conformal variational computations than are $\tau, \rho, R$. We put

$$
|V|^{2}=V_{i j} V_{i j}, \quad|C|^{2}=C_{i j k l} C_{i j k l}, \quad|L|^{2}=L_{a b} L_{a b}, \quad\langle L, G\rangle=L_{a b} G_{a b}
$$

and similarly for other tensor quantities. The Paneitz quantity and Paneitz operator are

$$
\begin{aligned}
& Q=-2|V|^{2}+(m / 2) J^{2}+\Delta J, \\
& P=\Delta^{2}+\delta\{(m-2) J-4 V \cdot\} d+\{(m-4) / 2\} Q,
\end{aligned}
$$

where $d$ is the exterior derivative, $\delta$ is the formal adjoint of $d$, and $V$. is the realization of $V$ as an endomorphism of the cotangent bundle $T^{*} M$.

Indices after a bar indicate covariant differentiations with respect to the LeviCivita connection of $g$, and indices after a colon indicate covariant differentiations with respect to the Levi-Civita connection of the boundary metric induced by $g$. Let $f$ be an indeterminant element of $C^{\infty}(M)$. We adopt the nonstandard abbreviations in Table 8.1. 
TABLE 8.1

\begin{tabular}{||c|c|c||c|c|c||}
\hline Abbr. & Invt. & Index expn. & Abbr. & Invt. & Index expn. \\
\hline$X_{1}$ & $N \tau$ & $R_{i j i j \mid N}$ & $Y_{5}(f)$ & $(N f) F$ & $f_{\mid N} R_{a N a N}$ \\
\hline$X_{2}$ & $\tau H$ & $R_{i j i j} L_{a a}$ & $Y_{6}(f)$ & $\langle\tilde{\nabla} \tilde{\nabla} f, L\rangle$ & $f_{: a b} L_{a b}$ \\
\hline$X_{3}$ & $F H$ & $R_{a N a N} L_{b b}$ & $Y_{7}(f)$ & $(N f)|L|^{2}$ & $f_{\mid N} L_{a b} L_{a b}$ \\
\hline$X_{4}$ & $\langle G, L\rangle$ & $R_{a N b N} L_{a b}$ & $Y_{8}(f)$ & $N(-\Delta) f$ & $f_{\mid i i N}$ \\
\hline$X_{5}$ & $\langle T, L\rangle$ & $R_{c a c b} L_{a b}$ & $Z_{1}(f, f)$ & $(N f) N^{2} f$ & $f_{\mid N} f_{\mid N N}$ \\
\hline$X_{6}$ & $H^{3}$ & $L_{a a} L_{b b} L_{c c}$ & $Z_{2}(f, f)$ & $(N f)(-\tilde{\Delta}) f$ & $f_{\mid N} f_{: a a}$ \\
\hline$X_{7}$ & $H|L|^{2}$ & $L_{a a} L_{b c} L_{b c}$ & $Z_{3}(f, f)$ & $(N f)^{2} H$ & $f_{\mid N} f_{\mid N} L_{a a}$ \\
\hline$X_{8}$ & $\operatorname{tr} L^{3}$ & $L_{a b} L_{b c} L_{c a}$ & $Z_{4}(f, f)$ & $|\tilde{d} f|^{2} H$ & $f_{: a} f_{: a} L_{b b}$ \\
\hline$Y_{1}(f)$ & $(N f) \tau$ & $f_{\mid N} R_{i j i j}$ & $Z_{5}(f, f)$ & $\langle\tilde{d} f \otimes \tilde{d} f, L\rangle$ & $f_{: a} f_{: b} L_{a b}$ \\
\hline$Y_{2}(f)$ & $\left(N^{2} f\right) H$ & $f_{\mid N N} L_{a a}$ & $Z_{6}(f, f)$ & $\langle\tilde{d} f, \tilde{d}(N f)\rangle$ & $f_{: a}\left(f_{\mid N}\right): a$ \\
\hline$Y_{3}(f)$ & $(-\tilde{\Delta} f) H$ & $f_{: a a} L_{b b}$ & $E_{1}(f, f, f)$ & $(N f)|\tilde{d} f|^{2}$ & $f_{\mid N} f_{: a} f_{: a}$ \\
\hline$Y_{4}(f)$ & $(N f) H^{2}$ & $f_{\mid N} L_{a a} L_{b b}$ & $E_{2}(f, f, f)$ & $(N f)^{3}$ & $f_{\mid N} f_{\mid N} f_{\mid N}$ \\
\hline
\end{tabular}

Let

$$
\begin{aligned}
S= & -\frac{1}{12} X_{1}+\frac{1}{6} X_{2}-X_{4}+\frac{1}{9} X_{6}-\frac{1}{3} X_{8}, \quad m=4, \\
\mathscr{S}(\omega)= & \frac{1}{3} Y_{1}(\omega)+Y_{3}(\omega)-Y_{5}(\omega)-Y_{6}(\omega)-\frac{1}{2} Y_{8}(\omega), \quad m=4, \\
\mathscr{L}_{4}= & -\frac{1}{m-1} X_{2}+X_{3}-(m-3) X_{4}+X_{5}, \\
\mathscr{L}_{5}= & -\frac{2}{3(m-1)} X_{6}+X_{7}-\frac{m-1}{3} X_{8}, \\
l_{1}(\omega)= & -Y_{4}(\omega)+(m-1) Y_{7}(\omega), \\
l_{2}(\omega)= & -Y_{1}(\omega)-(m-3) Y_{2}(\omega)+Y_{3}(\omega)-\frac{1}{m-1} Y_{4}(\omega)+(m-1) Y_{5}(\omega), \\
l_{3}(\omega)= & \frac{m^{2}-3 m-2}{m-1} Y_{2}(\omega)-\frac{2 m}{m-1} Y_{3}(\omega)-\frac{m^{2}-5 m+2}{(m-1)^{2}} Y_{4}(\omega) \\
& -(m-4) Y_{5}(\omega)+(m-2) Y_{6}(\omega)+Y_{8}(\omega), \\
\mathscr{A}= & X_{6}-(m-1)^{2} X_{8}, \\
q_{1}(\omega)= & Y_{3}(\omega)-(m-1) Y_{6}(\omega), \\
q_{2}(\omega)= & (m-3) Y_{1}(\omega)+(m-3)(m-2) Y_{2}(\omega)-2(m-2) Y_{3}(\omega), \\
q_{3}(\omega)= & (m-1) Y_{1}(\omega)-(m-1)(m-2) Y_{2}(\omega)+2(m-2) Y_{4}(\omega) .
\end{aligned}
$$

We note the identity

$$
f_{\mid i i N}=f_{\mid N N N}+\left(f_{\mid N}\right)_{: a a}+2\left(L_{a b} f_{: b}\right): a-H: a f_{: a}-F f_{\mid N}-|L|^{2} f_{\mid N}-H f_{\mid N N},
$$

and the local expression

$\chi(M)=\left(32 \pi^{2}\right)^{-1} \int_{M}\left(|C|^{2}+4 Q\right) d x+\left(4 \pi^{2}\right)^{-1} \oint_{\partial M}\left(S-\mathscr{L}_{4}-\mathscr{L}_{5}\right) d y, \quad m=4$,

for the Euler characteristic in dimension 4. 
b. Explicit zeta functions. As in $\S 7$, we adopt the convention that the index $j$ always runs over $\mathbb{N}$. $a$ will always be a positive real number. We note at the outset the integral formulas

$$
a^{-s}=\frac{1}{\Gamma(s)} \int_{0}^{\infty} t^{s-1} e^{-a t} d t, \quad \zeta_{a}(s)=\frac{1}{\Gamma(s)} \int_{0}^{\infty} t^{s-1} \frac{e^{-a t}}{1-e^{-t}} d t
$$

[WW, 12.2, 13.12], as well as

$$
\begin{gathered}
\zeta_{a}(0)=-a+\frac{1}{2}, \quad \zeta_{a}(-1)=-\frac{1}{2} a^{2}+\frac{1}{2} a-\frac{1}{12}, \\
\zeta_{a}(-2)=-\frac{1}{6} a(a-1)(2 a-1) .
\end{gathered}
$$

[WW, 13.14 and 7.2].

Our goal here is to establish formulas (7.5-7.7). We note that Vardi [V, Proposition 3.1] has calculated $\left(H_{d}^{n}\right)^{\prime}(0)$ for the functions

$$
H_{d}^{n}(s)=\sum_{k=1}^{\infty} \frac{k^{d}}{k^{s}(k+n)^{s}}, \quad d, n \in \mathbb{Z}^{+} .
$$

These can clearly be related to our double zeta functions; for example,

$$
\begin{gathered}
h_{1}=H_{0}^{1}, \quad f_{1}=2 H_{1}^{1}+H_{0}^{1}, \quad h_{1 / 2}(s)=4^{s} H_{0}^{2}(s)-H_{0}^{1}(s), \\
f_{1 / 2}(s)=4^{s}\left(H_{1}^{2}(s)+H_{0}^{2}(s)\right)-2 H_{1}^{1}(s)-H_{0}^{1}(s) .
\end{gathered}
$$

In fact, the $H_{d}^{n}$ with $d \in \mathbb{N}, n \in \mathbb{Z}^{+}$would be sufficient for our purposes. Since

$$
H_{d}^{n}(s-1)=H_{d+2}^{n}(s)+n H_{d+1}^{n}(s),
$$

knowledge of the behavior of the $H_{d}^{n}$ at $s=0$ leads to knowledge of the behavior the $H_{d}^{n}$, and thus the $h_{a}$ and $f_{a}$ for $a \in \frac{1}{2} \mathbb{N}$, at all nonpositive integers. More specifically, let $d$ range through $\mathbb{N}, n$ through $\{1,2\}$, and $a$ through $\frac{1}{2} \mathbb{N}$. If we know $H_{d}^{n}(0)$ and $\left(H_{d}^{n}\right)^{\prime}(0)$, then we also know $h_{a}(-m), h_{a}^{\prime}(-m)$, $f_{a}(-m), f_{a}^{\prime}(-m)$. The formulas derived below agree with the consequences of Vardi's formulas.

Consider the zeta function

$$
\begin{aligned}
f_{a}(s) & =\sum_{j}(2 j+2 a+1)[(j+a)(j+a+1)]^{-s} \\
& =\sum_{j}\left\{(j+a)^{-s}(j+a+1)^{1-s}+(j+a)^{1-s}(j+a+1)^{-s}\right\} .
\end{aligned}
$$

Applying the Mellin transform, we get

$$
f_{a}(s)=\frac{1}{\Gamma(s) \Gamma(s-1)} \int_{0}^{\infty} \int_{0}^{\infty}(u v)^{s-2}(u+v) \sum_{j} e^{-(j+a)(u+v)-v} d u d v
$$

Switching coordinates to $t=u+v, \theta=u / t$ (so that $u=\theta t, v=(1-\theta) t$ ), and noting that $d u \wedge d v=t d \theta \wedge d t$, we get

$$
f_{a}(s)=\frac{1}{\Gamma(s) \Gamma(s-1)} \int_{0}^{1}[\theta(1-\theta)]^{s-2}\left(\int_{0}^{\infty} t^{2 s-2} \frac{e^{-(1-\theta) t-a t}}{1-e^{-t}} d t\right) d \theta .
$$


We expand $e^{\theta t}$ in a power series, perform the $\theta$ integration, and use (8.1):

$$
\begin{aligned}
f_{a}(s) & =\frac{1}{\Gamma(s) \Gamma(s-1)} \sum_{k=0}^{\infty} \frac{1}{k !} c_{k}(s) I_{a, k}(s), \\
c_{k}(s) & :=\int_{0}^{1}[\theta(1-\theta)]^{s-2} \theta^{k} d \theta=\frac{\Gamma(s-1) \Gamma(s+k-1)}{\Gamma(2 s+k-2)}, \quad k \in \mathbb{N}, \\
I_{a, k}(s) & :=\int_{0}^{\infty} t^{2 s-2+k} \frac{e^{-(a+1) t}}{1-e^{-t}} d t=\Gamma(2 s-1+k) \zeta_{a+1}(2 s-1+k) .
\end{aligned}
$$

Thus

$$
f_{a}(s)=\frac{1}{\Gamma(s)} \sum_{k=0}^{\infty} d_{k}(s) \zeta_{a+1}(2 s+k-1), \quad d_{k}(s):=\frac{(2 s+k-2) \Gamma(s+k-1)}{k !} .
$$

(7.6) is immediate from this. The only singularity of $\zeta_{a+1}(z)$ is a simple pole with residue 1 at $z=1$ [WW, 13.13]; in calculating $f_{a}^{\prime}(0)$, we encounter this pole at the $k=2$ term; in calculating $f_{a}^{\prime}(-1)$, at the $k=4$ term. For the $s=0$ calculation, we note that

$$
\begin{aligned}
& d_{0}(s)=2 \Gamma(s), \quad d_{1}(s)=(2 s-1) \Gamma(s), \quad d_{2}(s)=s^{2} \Gamma(s), \\
& d_{k}(0)=\frac{k-2}{k(k-1)}, \quad k \geq 3 .
\end{aligned}
$$

This allows us to write

$$
\begin{gathered}
f_{a}^{\prime}(0)=4 \zeta_{a+1}^{\prime}(-1)-2 \zeta_{a+1}^{\prime}(0)-2 a-\frac{1}{2}+\mathscr{I}_{3} \\
\mathscr{I}_{3}:=\sum_{k=3}^{\infty} \frac{k-2}{k(k-1)} \zeta_{a+1}(k-1)=\sum_{k=3}^{\infty}\left(\frac{1}{(k-1) !}-\frac{2}{k !}\right) \int_{0}^{\infty} t^{k-2} \frac{e^{-(a+1) t}}{1-e^{-t}} d t
\end{gathered}
$$

But

$$
\begin{aligned}
\sum_{k=3}^{\infty}\left(\frac{1}{(k-1) !}-\frac{2}{k !}\right) t^{k} & =t\left(e^{t}-1-t\right)-2\left(e^{t}-1-t-\frac{1}{2} t^{2}\right) \\
& =(t-2)\left(e^{t}-1\right)+2 t
\end{aligned}
$$

Thus

$$
\begin{aligned}
\mathscr{I}_{3} & =\int_{0}^{\infty}\left(t^{-1} e^{-a t}-2 t^{-2} e^{-a t}+2 t^{-1} \frac{e^{-(a+1) t}}{1-e^{-t}}\right) d t \\
= & \left.\frac{d}{d s}\right|_{s=0}\left\{\frac{1}{\Gamma(s)} \int_{0}^{\infty} t^{s-1} e^{-a t} d t-\frac{2}{\Gamma(s)} \int_{0}^{\infty} t^{s-2} e^{-a t} d t\right. \\
& \left.\quad+\frac{2}{\Gamma(s)} \int_{0}^{\infty} t^{s-1} \frac{e^{-(a+1) t}}{1-e^{-t}} d t\right\} \\
& =\left.\frac{d}{d s}\right|_{s=0}\left\{a^{-s}-\frac{2 a^{1-s}}{s-1}+2 \zeta_{a+1}(s)\right\} \\
& =-(2 a+1) \log a+2 a+2 \zeta_{a+1}^{\prime}(0) .
\end{aligned}
$$

(Note that in the first line directly above, the integral converges at $t=0$, even though the integrals of the individual terms do not.) This gives

$$
f_{a}^{\prime}(0)=4 \zeta_{a+1}^{\prime}(-1)-\frac{1}{2}-(2 a+1) \log a
$$


as desired for (7.7). To compute $f_{a}^{\prime}(-1)$, note that

$$
\begin{gathered}
d_{3}(s)=\frac{s(s+1)(2 s+1) \Gamma(s)}{6}, \quad d_{4}(s)=\frac{s(s+1)^{2}(s+2) \Gamma(s)}{12}, \\
d_{k}(-1)=\frac{k-4}{k(k-1)(k-2)}, \quad k \geq 5
\end{gathered}
$$

so

$$
\begin{aligned}
f_{a}^{\prime}(-1)= & 4 \zeta_{a+1}^{\prime}(-3)-6 \zeta_{a}^{\prime}(-2)+2 \zeta_{a}^{\prime}(-1)+2 \zeta_{a+1}(-2) \\
& -2 \zeta_{a+1}(-1)+\frac{1}{6} \zeta_{a+1}(0)-\frac{1}{24}-\mathscr{I}_{5}
\end{aligned}
$$

where

$$
\begin{aligned}
\mathscr{I}_{5} & :=\sum_{k=5}^{\infty} \frac{k-4}{k(k-1)(k-2)} \zeta_{a+1}(k-3) \\
& =\sum_{k=5}^{\infty}\left(\frac{1}{(k-2) !}-\frac{6}{(k-1) !}+\frac{12}{k !}\right) \int_{0}^{\infty} t^{k-4} \frac{e^{-(a+1) t}}{1-e^{-t}} d t
\end{aligned}
$$

But

$$
\sum_{k=5}^{\infty}\left(\frac{1}{(k-2) !}-\frac{6}{(k-1) !}+\frac{12}{k !}\right) t^{k}=\left(t^{2}-6 t+12\right)\left(e^{t}-1\right)-12 t
$$

and thus

$$
\begin{aligned}
\mathscr{I}_{5}= & \int_{0}^{\infty}\left(\left(t^{-2}-6 t^{-3}+12 t^{-4}\right) e^{-a t}-12 t^{-3} \frac{e^{-(a+1) t}}{1-e^{-t}}\right) d t \\
= & \left.\frac{d}{d s}\right|_{s=0}\left\{\frac{1}{\Gamma(s)} \int_{0}^{\infty} t^{s-2} e^{-a t} d t-\frac{6}{\Gamma(s)} \int_{0}^{\infty} t^{s-3} e^{-a t} d t\right. \\
& \left.\quad+\frac{12}{\Gamma(s)} \int_{0}^{\infty} t^{s-4} e^{-a t} d t-\frac{12}{\Gamma(s)} \int_{0}^{\infty} t^{s-3} \frac{e^{-(a+1) t}}{1-e^{-t}} d t\right\} \\
= & \left.\frac{d}{d s}\right|_{s=0}\left\{\frac{a^{1-s}}{s-1}-\frac{6 a^{2-s}}{(s-1)(s-2)}+\frac{12 a^{3-s}}{(s-1)(s-2)(s-3)}\right. \\
= & a(a+1)(2 a+1) \log a-a-\frac{9}{2} a^{2}-\frac{11}{3} a^{3}-6 \zeta_{a+1}^{\prime}(-2)-9 \zeta_{a+1}(-2) .
\end{aligned}
$$

This gives

$$
\begin{aligned}
f_{a}^{\prime}(-1)= & 4 \zeta_{a+1}^{\prime}(-3)+2 \zeta_{a+1}^{\prime}(-1)+11 \zeta_{a+1}(-2)-2 \zeta_{a+1}(-1) \\
& +\frac{11}{3} a^{3}+\frac{9}{2} a^{2}+\frac{5}{6} a-\frac{1}{8}-a(a+1)(2 a+1) \log a .
\end{aligned}
$$

By (8.2),

$$
f_{a}^{\prime}(-1)=4 \zeta_{a+1}^{\prime}(-3)+2 \zeta_{a+1}^{\prime}(-1)-a(a+1)(2 a+1) \log a+\frac{1}{24},
$$

as desired for (7.7). 
Now consider

$$
\begin{aligned}
h_{a}(s) & =\sum_{j}[(j+a)(j+a+1)]^{-s} \\
& =\frac{1}{\Gamma(s)^{2}} \int_{0}^{\infty} \int_{0}^{\infty}(u v)^{s-1} \sum_{j} e^{-(j+a)(u+v)-v} d u d v \\
& =\frac{1}{\Gamma(s)^{2}} \int_{0}^{1}[\theta(1-\theta)]^{s-1}\left(\int_{0}^{\infty} t^{2 s-1} e^{\theta t} \frac{e^{-(a+1) t}}{1-e^{-t}} d t\right) d \theta \\
& =\frac{1}{\Gamma(s)^{2}} \sum_{k=0}^{\infty} c_{k}(s+1) I_{a, k}\left(s+\frac{1}{2}\right) \\
& =\frac{1}{\Gamma(s)} \sum_{k=0}^{\infty} e_{k}(s) \zeta_{a+1}(2 s+k),
\end{aligned}
$$

where

$$
e_{k}(s):=\Gamma(s+k) / k !
$$

(7.5) is immediate from this; the first term on the right in (7.5) is produced by the singularity of $\zeta_{a+1}$ at $s=1$. In calculating $h_{a}^{\prime}(0)$, we encounter the singularity of $\zeta_{a+1}$ at the $k=1$ term; in calculating $h_{a}^{\prime}(-1)$, at the $k=3$ term.

For the $s=0$ calculation, note that

$$
\begin{aligned}
& e_{0}(s)=\Gamma(s), \quad e_{1}(s)=s \Gamma(s), \\
& e_{k}(0)=1 / k, \quad k \geq 2 \text {. }
\end{aligned}
$$

Thus

$$
h_{a}^{\prime}(0)=2 \zeta_{a+1}^{\prime}(0)+\alpha(a)+I_{2}
$$

where $\alpha(a)$ is defined by

$$
\zeta_{a+1}(1+s)=1 / s+\alpha(a)+O(s),
$$

and

$$
\begin{aligned}
\mathscr{I}_{2} & :=\sum_{k=2}^{\infty} \frac{1}{k} \zeta_{a+1}(k)=\sum_{k=2}^{\infty} \frac{1}{k !} \int_{0}^{\infty} t^{k-1} \frac{e^{-(a+1) t}}{1-e^{-t}} d t \\
& =\int_{0}^{\infty}\left(e^{t}-1-t\right) t^{-1} \frac{e^{-(a+1) t}}{1-e^{-t}} d t \\
& =\int_{0}^{\infty}\left(t^{-1} e^{-a t}-\frac{e^{-(a+1) t}}{1-e^{-t}}\right) d t \\
& =\left.\frac{d}{d s}\right|_{s=0}\left\{\frac{1}{\Gamma(s)} \int_{0}^{\infty} t^{s-1} e^{-a t} d t-\frac{1}{\Gamma(s)} \int_{0}^{\infty} t^{s} \frac{e^{-(a+1) t}}{1-e^{-t}} d t\right\} \\
& =\left.\frac{d}{d s}\right|_{s=0}\left\{a^{-s}-s \zeta_{a+1}(s+1)\right\} \\
& =-\log a-\alpha(a) .
\end{aligned}
$$

This gives

as desired for (7.7).

$$
h_{a}^{\prime}(0)=2 \zeta_{a+1}^{\prime}(0)-\log a
$$


For the $s=-1$ calculation, note that

$$
\begin{array}{ll}
e_{2}(s)=\frac{1}{2} s(s+1) \Gamma(s), & e_{3}(s)=\frac{1}{6} s(s+1)(s+2) \Gamma(s), \\
e_{k}(-1)=1 / k(k-1), & k \geq 4 .
\end{array}
$$

Thus

$$
h_{a}^{\prime}(-1)=2 \zeta_{a+1}^{\prime}(-2)-2 \zeta_{a+1}^{\prime}(-1)+\zeta_{a+1}(-1)-\frac{1}{2} \zeta_{a+1}(0)-\frac{1}{6} \alpha(a)-\mathscr{I}_{4},
$$

where

$$
\begin{aligned}
\mathscr{I}_{4} & :=\sum_{k=4}^{\infty} \frac{k-2}{k !} \int_{0}^{\infty} t^{k-3} \frac{e^{-(a+1) t}}{1-e^{-t}} d t \\
& =\int_{0}^{\infty}\left\{\left(t^{-2}-2 t^{-3}\right)\left(e^{t}-1\right)+2 t^{-2}-\frac{1}{6}\right\} \frac{e^{-(a+1) t}}{1-e^{-t}} d t \\
& =\left.\frac{d}{d s}\right|_{s=0}\left\{\frac{a^{1-s}}{s-1}-\frac{2 a^{2-s}}{(s-1)(s-2)}+\frac{2}{s-1} \zeta_{a+1}(s-1)-\frac{1}{6} s \zeta_{a+1}(s+1)\right\} \\
& =-a+a \log a-\frac{3}{2} a^{2}+a^{2} \log a-2 \zeta_{a+1}(-1)-2 \zeta_{a+1}^{\prime}(-1)-\frac{1}{6} \alpha(a) .
\end{aligned}
$$

The total is

$$
h_{a}^{\prime}(-1)=2 \zeta_{a+1}^{\prime}(-2)+3 \zeta_{a+1}(-1)-\frac{1}{2} \zeta_{a+1}(0)-\left(a^{2}+a\right) \log a+a+\frac{3}{2} a^{2}
$$

by $(8.2)$,

$$
h_{a}^{\prime}(-1)=2 \zeta_{a+1}^{\prime}(-2)-\left(a^{2}+a\right) \log a,
$$

as desired for (7.7).

\section{REFERENCES}

[A] D. R. Adams, A sharp inequality of $J$. Moser for higher order derivatives, Ann. of Math. (2) 128 (1988), 385-398.

[Be] W. Beckner, Sharp Sobolev inequalities on the sphere and the Moser-Trudinger inequality, Ann. of Math. 138 (1993), 213-242.

[Bl] D. Bleecker, Determination of a Riemannian metric from the first variation of its spectrum, Amer. J. Math. 107 (1985), 815-831.

[Br1] T. Branson, Conformally covariant equations on differential forms, Comm. Partial Differential Equations 7 (1982), 393-431.

[Br2] _ Differential operators canonically associated to a conformal structure, Math. Scand. 57 (1985), 293-345.

[Br3] $\longrightarrow$ Group representations arising from Lorentz conformal geometry, J. Funct. Anal. 74 (1987), 199-291.

[Br4] _- Harmonic analysis in vector bundles associated to the rotation and spin groups, $\mathrm{J}$. Funct. Anal. 106 (1992), 314-328.

[BCY] T. Branson, S.-Y.A. Chang, and P. Yang, Estimates and extremals for zeta function determinants on four-manifolds, Comm. Math. Phys. 149 (1992), 241-262.

[BG] T. Branson and P. Gilkey, The asymptotics of the Laplacian on a manifold with boundary, Comm. Partial Differential Equations 15 (1990), 245-272.

[BØ1] T. Branson and B. Ørsted, Conformal indices of Riemannian manifolds, Compositio Math. 60 (1986), 261-293. 
[BØ2] __, Conformal geometry and global invariants, Differential Geom. Appl. 1 (1991), 279308.

[BØ3] _ Explicit functional determinants in four dimensions, Proc. Amer. Math. Soc. 113 (1991), 669-682.

[CT] C. Callias and C. Taubes, Functional determinants in Euclidean Yang-Mills theory, Comm. Math. Phys. 77 (1980), 229-250.

[ES] M. Eastwood and M. Singer, A conformally invariant Maxwell gauge, Phys. Lett. A 107 (1985), 73-74.

[E1] J. Escobar, Sharp constant in a Sobolev trace inequality, Indiana Univ. Math. J. 37 (1988), 687-698.

[E2] $\_$The Yamabe problem on manifolds with boundary, J. Differential Geom. 35 (1992), 21-84.

[FG] H. D. Fegan and P. Gilkey, Invariants of the heat equation, Pacific J. Math. 117 (1985), 233-254.

[G1] P. Gilkey, Recursion relations and the asymptotic behavior of the eigenvalues of the Laplacian, Compositio Math. 38 (1979), 201-240.

[G2] - Invariant theory, the heat equation, and the Atiyah-Singer index theorem, Publish or Perish, Wilmington, DE, 1984.

[GS] P. Gilkey and L. Smith, The eta invariant for a class of elliptic boundary value problems, Comm. Pure Appl. Math. 36 (1983), 85-131.

[K1] Y. Kosmann, Dérivées de Lie des spineurs, Ann. Mat. Pura Appl. (4) 91 (1972), 317-395.

[K2] _ Degrés conformes des laplaciens et des opérateurs de Dirac, C. R. Acad. Sci. Paris Ser. I Math. 280 (1975), 283-285.

[O] E. Onofri, On the positivity of the effective action in a theory of random surfaces, Comm. Math. Phys. 86 (1982), 321-326.

[Ø] B. Ørsted, The conformal invariance of Huygens' principle, J. Differential Geom. 16 (1981), 1-9.

[OPS1] B. Osgood, R. Phillips, and P. Sarnak, Extremals of determinants of Laplacians, J. Funct. Anal. 80 (1988), 148-211.

[OPS2] _ Compact isospectral sets of surfaces, J. Funct. Anal. 80 (1988), 212-234.

[P] S. Paneitz, A quartic conformally covariant differential operator for arbitrary pseudoRiemannian manifolds, preprint, 1983.

[R] S. Rosenberg, The determinant of a conformally covariant operator, J. London Math. Soc. 36 (1987), 553-568.

[S] R. Strichartz, Linear algebra of curvature tensors and their covariant derivatives, Canad. J. Math. 40 (1988), 1105-1143.

[V] I. Vardi, Determinants of Laplacians and multiple gamma functions, SIAM J. Math. Anal. 19 (1988), 493-507.

[W] W. Weisberger, Normalization of the path integral measure and the coupling constants for bosonic strings, Nuclear Phys. B 284 (1987), 171-200.

[WW] E. T. Whittaker and G. N. Watson, $A$ course of modern analysis, 4th ed., Cambridge Univ. Press, London and New York, 1946.

Department of Mathematics, University of Iowa, Iowa City, Iowa 52242

E-mail address: branson@math.uiowa.edu

IMFUFA, Roskilde University Center, DK-4000 Roskilde, Denmark

Department of Mathematics, University of Oregon, Eugene, Oregon 97403

E-mail address: gilkey@bright .math.uoregon.edu 